\title{
UM MÉTODO DE RESTRIÇÕES ATIVAS \\ PARA MINIMIZAÇÃO EM CAIXAS
}

\author{
Marina Andretta
}

Dissertação apresentada

$\mathrm{aO}$

Instituto de Matemática e Estatística

da

Universidade de São Paulo

para

obtenção do grau

de

Mestre em Ciência da Computação

Área de Concentração: Ciência da Computação

Orientador: Prof. Dr. Ernesto G. Birgin

São Paulo, Março de 2004 


\title{
UM MÉTODO DE RESTRIÇÕES ATIVAS PARA MINIMIZAÇÃO EM CAIXAS
}

\author{
Este exemplar corresponde à redação final \\ da dissertação de mestrado devidamente \\ corrigida e defendida por \\ Marina Andretta \\ e aprovada pela comissão julgadora.
}

São Paulo, Março de 2004.

Banca examinadora:

- Prof. Dr. Ernesto G. Birgin (Orientador) - IME-USP

- Prof. Dr. Paulo José da Silva e Silva - IME-USP

- Prof. Dr. Roberto Andreani - IMECC-UNICAMP 


\section{Sumário}

Introdução

1 O método Moré-Sorensen para minimização de quadráticas em bolas 4

1.1 Fundamentação teórica . . . . . . . . . . . . . . . . . . 5

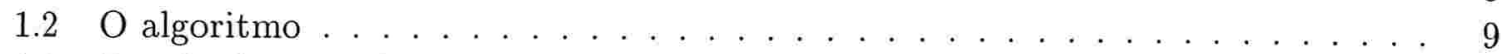

1.3 Resultados numéricos . . . . . . . . . . . . . . . . . 21

2 Regiões de confiança $\quad 26$

$2.1 \mathrm{O}$ algoritmo baseado em regiões de confiança . . . . . . . . . . . . . 26

2.2 Resultados numéricos . . . . . . . . . . . . . . . . . . 30

3 O método do Gradiente Espectral Projetado 32

3.1 O algoritmo . . . . . . . . . . . . . . . . . . . . . . . 32

3.2 Implementação . . . . . . . . . . . . . . . . . . . . . . . . . . . . 34

4 Um método híbrido para minimização nas faces $\quad 37$

$4.1 \quad \mathrm{O}$ algoritmo . . . . . . . . . . . . . . . . . . . . . . . . 38

4.2 Teorema de convergência . . . . . . . . . . . . . . . . . . . . . . . . . . . . . . .

4.3 Implementação . . . . . . . . . . . . . . . . . . . . . . . . . . . . 41

5 Um método de restrições ativas para minimização em caixas 44

$5.1 \mathrm{O}$ algoritmo . . . . . . . . . . . . . . . . . . . . . . 44

5.2 Teorema de convergência . . . . . . . . . . . . . . . . . . 46

5.3 Implementação . . . . . . . . . . . . . . . . . . . . . . . . . . . . 47

5.4 Resultados numéricos . . . . . . . . . . . . . . . . . . 48

Conclusões e trabalho futuro $\quad 55$

A Provas dos lemas do capítulo $1 \quad 56$

$\begin{array}{ll}\text { Referências bibliográficas } & 73\end{array}$ 


\section{Introdução}

O problema considerado neste trabalho consiste na minimização de uma função $f: \mathbb{R}^{n} \rightarrow \mathbb{R}$ suave com limitantes nas variáveis. O conjunto factível $\Omega$ é definido por $\Omega=\left\{x \in \mathbb{R}^{n} \mid l \leq\right.$ $x \leq u\}$. Algoritmos para minimização em caixas são usados como subalgoritmos para resolver os subproblemas que aparecem em vários métodos de Lagrangeano aumentado e métodos de penalização para otimização com restrições gerais.

Os métodos de restrições ativas para a minimização de funções com restrições de caixa baseiam-se no seguinte princípio: se conhecemos quais restrições são satisfeitas por igualdade na solução (ativas) e quais não (inativas) podemos fixar algumas variáveis nos seus limitantes e resolver um problema irrestrito nas outras variáveis (variáveis livres). Por isso, os métodos de restrições ativas tentam, a cada iteração, inferir quais restrições serão ativas na solução e resolver um problema localmente irrestrito nas variáveis livres.

Recentemente, foi introduzido um novo método de restrições ativas para a minimização de problemas com restrições de caixa [2]. O algoritmo combina um método irrestrito com Gradiente Espectral Projetado para eliminar restrições do conjunto das variáveis livres. $\mathrm{O}$ algoritmo irrestrito inclui uma busca linear que tenta acrescentar muitas restrições no conjunto de restrições ativas a cada iteração. Dentro das faces, utiliza-se um método do tipo Newton truncado que, desenhado para problemas de grande porte, aproxima os produtos hessianavetor por quocientes incrementais.

Experimentos computacionais mostraram que esta alternativa não é a mais adequada para problemas de pequeno e médio porte, com hessianas esparsas ou com gradientes computacionalmente custosos. No presente trabalho, estudaremos os métodos de restrições ativas, em particular o proposto em [2], e proporemos e implementaremos uma alternativa para o método utilizado na minimização dentro das faces.

O método alternativo escolhido para a minimização dentro das faces é o método de regiões de confiança combinado com o método do Gradiente Espectral Projetado. Para resolver os subproblemas que aparecem a cada iteração do método de regiões de confiança foi escolhido o método Moré-Sorensen para minimização de quadráticas em bolas descrito em [18]. O método Moré-Sorensen é interessante porque resultados numéricos mostram que métodos que aproximam o minimizador da quadrática na bola (como Dogleg ou a estratégia de somar uma matriz diagonal para contornar o caso de matrizes não positivas definidas) apresentam piores resultados do que os obtidos pela minimização exata (feita pelo método Moré-Sorensen), apesar deste último utilizar mais de uma fatoração de matriz (veja [10] e [1]). 
O presente trabalho está organizado como segue. No capítulo 1 tratamos do método apresentado em [18] para a resolução do subproblema que aparece em cada iteração da técnica de regiões de confiança, ou seja, minimizar uma quadrática restrita a uma bola. Analisamos todo o desenvolvimento teórico do método, explicando as propriedades e características do problema a ser resolvido. Reproduzimos todos os lemas apresentados em [18] e enunciamos como novos lemas algumas afirmações de [18] que não nos pareceram triviais. No Apêndice, provamos todos lemas (tanto os de [18] como os por nós enunciados) passo a passo. Apresentamos o algoritmo para a resolução do problema. Por fim, apresentamos os resultados numéricos obtidos pela aplicação da nossa implementação deste algoritmo aos testes propostos em [18].

No capítulo 2 detalhamos a técnica de regiões de confiança e apresentamos um algoritmo para minimização de funções irrestritas que utiliza o método apresentado no capítulo 1 para resolver o subproblema que aparece em cada iteração (tal como sugerido em [18]). Apresentamos os resultados numéricos que nossa implementação deste algoritmo obteve quando aplicado às 18 funções de teste utilizadas em [18] e introduzidas em [17].

No capítulo 3 apresentamos o método do Gradiente Espectral Projetado. No capítulo 4 propomos um método híbrido para minimização nas faces, que mistura iterações dos métodos apresentados nos capítulos 2 e 3 . Enunciamos e provamos o teorema de convergência deste método e apresentamos detalhes de nossa implementação.

No capítulo 5 apresentamos o método de restrições ativas para minimização em caixas, que chamamos de BETRA, baseado no método introduzido em [2] e que utiliza o método do Gradiente Espectral Projetado para sair das faces e o algoritmo proposto no capítulo 4 para trabalhar nas faces. Enunciamos e provamos o teorema de convergência de BETRA. Apresentamos, ainda, detalhes de nossa implementação de BETRA e os resultados numéricos de sua aplicação na resolução dos problemas da coleção CUTE (veja [6]). Comparamos seu desempenho com o desempenho de LANCELOT (um conhecido método para a resolução de problemas com restrições de caixa, veja [8]) e constatamos que BETRA mostra-se competitivo, obtendo melhores índices de eficiência e robustez quando comparado com LANCELOT. Apresentamos os resultados obtidos.

Por fim, apresentamos as conclusões e idéias para trabalhos futuros. 


\section{Capítulo 1}

\section{O método Moré-Sorensen para minimização de quadráticas em bolas}

Métodos baseados em regiões de confiança para minimização de uma função $f$ qualquer seguem o seguinte princípio: a cada iteração $k$, dado um ponto $x^{k}$ e um raio $\Delta^{k}$ da região, minimiza-se uma aproximação quadrática da função $f$ no ponto $x^{k}$ restrita a esta região de confiança (ou, $\left\|x^{k}\right\| \leq \Delta^{k}$ ).

Ou seja, a cada iteração estamos interessados em resolver problemas do tipo

$$
\begin{array}{ll}
\operatorname{minimizar} & \varphi(w) \equiv \frac{1}{2} w^{T} B w+g^{T} w \\
\text { sujeita a } & \|w\| \leq \Delta^{k}
\end{array}
$$

onde $\Delta^{k}$ é um número positivo, $\|$.$\| é a norma euclidiana em \mathbb{R}^{n}, g \in \mathbb{R}^{n}$ e $B \in \mathbb{R}^{n \times n}$ é uma matriz simétrica.

Existem vários métodos que fornecem uma solução aproximada para (1.1). No entanto, estamos interessados em soluções exatas, ou pelo menos muito próximas disso.

O método proposto em [18] para resolução deste problema tem justamente este objetivo: encontrar a solução "exata" para (1.1). Para isso, faz-se uso do fato de que a solução $p^{k}$ de (1.1) é solução de um sistema do tipo $(B+\lambda I) p^{k}=-g$, com $\lambda \geq 0, \lambda\left(\Delta^{k}-\left\|p^{k}\right\|\right)=0$ e $B+\lambda I$ semidefinida positiva. Assim, o método tenta, em linhas gerais, encontrar um valor de $\lambda \geq 0$ que faça $B+\lambda I$ ser definida positiva e resolve o sistema, encontrando $p^{k}$. Se $\lambda=0$ e $\left\|p^{\bar{k}}\right\| \leq \Delta^{k}$ já temos a solução de (1.1). Caso contrário, o valor de $\lambda$ varia até que se encontre $\left\|p^{k}\right\|=\Delta^{k}$.

O método proposto em [18] tem alguns detalhes que serão vistos ao longo deste capítulo. Implementamos este método e fizemos os testes propostos em [18], verificando que os resultados obtidos pela nossa implementação foram equivalentes aos apresentados por este artigo. Isso é um indicador de que nossa implementação está correta. 
Neste capítulo veremos as propriedades do problema (1.1) e as dificuldades que aparecem em alguns casos. Reproduzimos os lemas de [18] e transformamos em lemas algumas afirmações de [18] que não nos pareceram evidentes. Depois de analisadas as características da solução do problema (1.1), é apresentado o desenvolvimento do algoritmo para a resolução deste problema e sua versão final. Por fim, apresentamos os resultados obtidos pela nossa implementação deste algoritmo aplicados aos testes propostos em [18].

\subsection{Fundamentação teórica}

A primeira coisa a se saber a respeito do problema (1.1) são os seguintes lemas (todas as provas dos lemas apresentados neste capítulo estão no Apêndice):

Lema 1.1.1 (Lema 2.1 de [18]) Se p é uma solução para (1.1) então p é uma solução para uma equação da forma

$$
(B+\lambda I) p=-g,
$$

com $B+\lambda I$ semidefinida positiva, $\lambda \geq 0$ e $\lambda(\Delta-\|p\|)=0$.

Prova : ver Apêndice.

Lema 1.1.2 (Lema 2.3 de [18]) Sejam $\lambda \in \mathbb{R}$ e $p \in \mathbb{R}^{n}$ satisfazendo (1.2) com $B+\lambda I$ semidefinida positiva.

(i) Se $\lambda=0$ e $\|p\| \leq \Delta$ então $p$ é solução de (1.1);

(ii) $p$ é solução de $\min \{\varphi(w):\|w\| \leq\|p\|\}$;

(iii) se $\lambda \geq 0$ e $\|p\|=\Delta$ então $p$ é solução de (1.1).

Se $B+\lambda I$ é definida positiva, $p$ é único em (i), (ii) e (iii).

Prova : ver Apêndice.

Note que, se $B$ for definida positiva, $B$ é inversível. Tomando-se $\lambda=0$, temos que, se $\left\|B^{-1} g\right\| \leq \Delta$ então $p=-B^{-1} g$ é solução de (1.1) (pelo Lema 1.1.2-(i)). Em outras palavras, se o passo de Newton $p$ está dentro da região delimitada por $D=\{w:\|w\| \leq \Delta\}$, podemos dar este passo e encontrar a solução de (1.1).

Mais ainda:

Lema 1.1.3 O problema (1.1) não tem solução na borda de $D=\{w:\|w\| \leq \Delta\}$ se e somente se $B$ é definida positiva e $\left\|B^{-1} g\right\|<\Delta$. 
Prova : ver Apêndice.

Ou seja, o Lema 1.1.3 nos diz que, se a solução de (1.1) não for o passo de Newton, então a solução deverá estar na fronteira de $D$.

Assim, quando $B$ não é definida positiva ou o passo de Newton está fora da região $D$, o Lema 1.1.1 sugere que busquemos uma solução $p$ de (1.2) com $\lambda \geq 0$ e $B+\lambda I$ semidefinida positiva. Como sabemos que, neste caso, a solução está na borda de $D$ (ou seja, $\|p\|=\Delta$ ), vale que $\lambda(\Delta-\|p\|)=0$. Além disso, o Lema 1.1.2-(iii) garante que $p$ é a solução de (1.1).

Ora, se tivermos um $\lambda \geq 0$ que faz $B+\lambda I$ semidefinida positiva, é fácil ver que existe um $\lambda^{*}$ maior ou igual a este $\lambda$ que faz $B+\lambda^{*} I$ definida positiva.

Vamos definir a função de uma variável $p(\lambda)$ como

$$
p(\lambda)=-(B+\lambda I)^{-1} g,
$$

para $\lambda \neq-\lambda_{j}$, onde $\lambda_{j}$ são os autovalores da matriz $B$.

Lembre-se de que estamos interessados na função $p(\lambda)$ apenas para valores de $\lambda>-\lambda$, ou seja, para valores de $\lambda$ que façam $B+\lambda I$ definida positiva. Se, neste intervalo, encontrarmos um $\lambda^{*} \geq 0$ tal que $\left\|p\left(\lambda^{*}\right)\right\|=\Delta$ está claro que teremos uma solução de (1.1).

De fato esta solução sempre existe. Para verificar isso, olhemos mais de perto para a função $p(\lambda)$.

Lema 1.1.4 Seja $p(\lambda)=-(B+\lambda I)^{-1} g$ com $B+\lambda I$ definida positiva.

Tome a decomposição $B=Q \Lambda Q^{T}, \operatorname{com} \Lambda=\operatorname{diag}\left(\lambda_{1}, \lambda_{2}, \ldots, \lambda_{n}\right)$ e $Q^{T} Q=I$, onde $\lambda_{1} \leq$ $\lambda_{2} \leq \ldots \leq \lambda_{n}$ são os autovalores de $B$ (que existe porque $B$ é simétrica).

Então, para $\lambda \neq-\lambda_{j}$,

$$
p(\lambda)=-\sum_{j=1}^{n} \frac{q_{j}^{T} g}{\lambda_{j}+\lambda} q_{j},
$$

onde $q_{j}$ é a $j$-ésima coluna de $Q$.

Prova : ver Apêndice.

Pelo Lema 1.1.4 podemos ver que o quadrado da norma euclidiana função $p(\lambda)$ é dado por

$$
\|p(\lambda)\|^{2}=\left(\sum_{j=1}^{n} \frac{q_{j}^{T} g}{\lambda_{j}+\lambda} q_{j}\right)^{T}\left(\sum_{j=1}^{n} \frac{q_{j}^{T} g}{\lambda_{j}+\lambda} q_{j}\right) .
$$

Como $Q^{T} Q=I$, temos que $\left(q_{i}\right)^{T} q_{i}=1$ para todo $i$ e $\left(q_{j}\right)^{T} q_{i}=0$ para todo $j \neq i$.

Assim, 


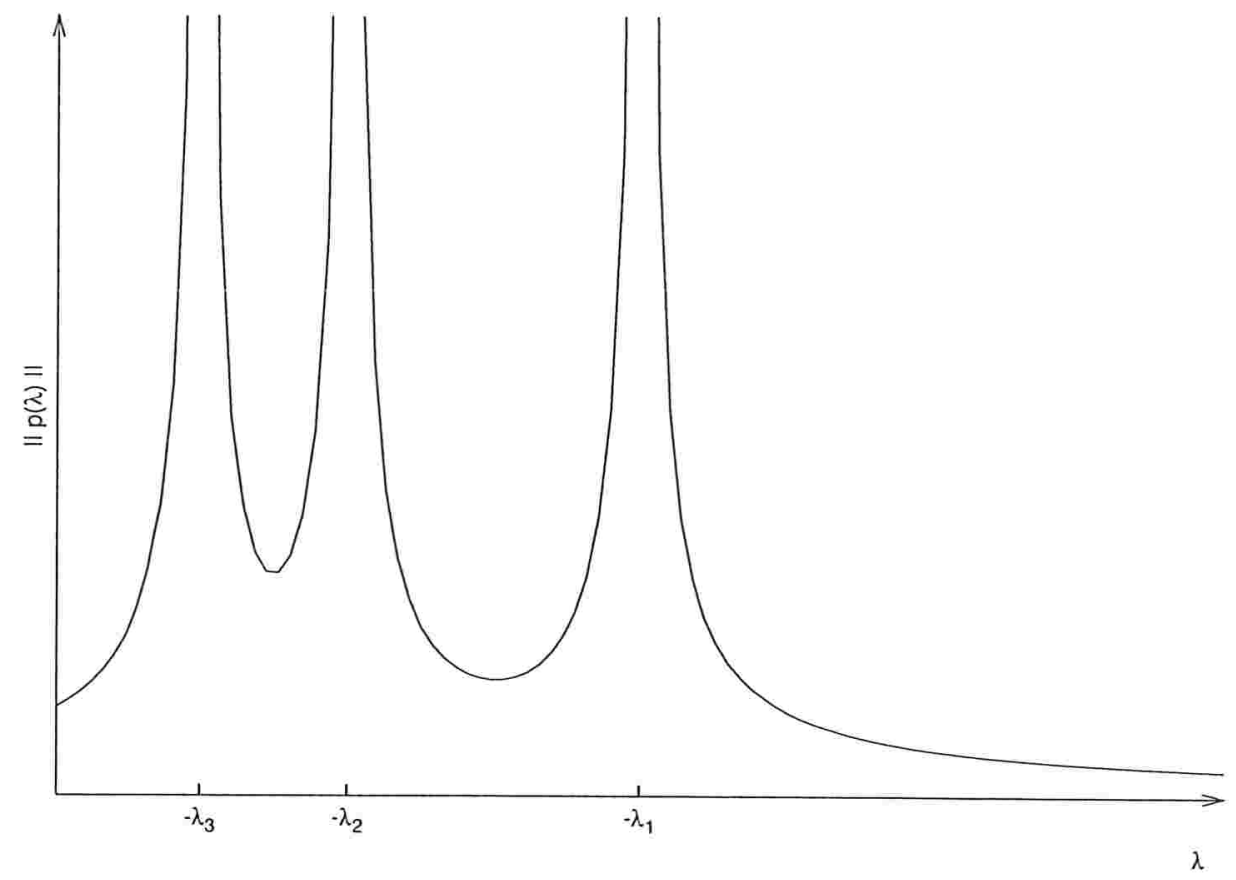

Figura 1.1: caso em que $q_{1}^{T} g \neq 0$

$$
\|p(\lambda)\|^{2}=\sum_{j=1}^{n} \frac{\left(q_{j}^{T} g\right)^{2}}{\left(\lambda_{j}+\lambda\right)^{2}}
$$

Note que, como a norma é positiva e $\Delta>0$, quando usamos relações do tipo $\|p(\lambda)\|=\Delta$, podemos também usar $\|p(\lambda)\|^{2}=\Delta^{2}$.

Observe que

$$
\lim _{\lambda \rightarrow \infty}\|p(\lambda)\|^{2}=0
$$

e, $\operatorname{para} q_{j}^{T} g \neq 0$

$$
\lim _{\lambda \rightarrow-\lambda_{j}}\|p(\lambda)\|^{2}=\infty
$$

Veja na figura 1.1 um desenho da função de $\|p(\lambda)\|$ para $q_{j}^{T} g \neq 0$.

Note que, se $\lambda>-\lambda_{1}$ temos que $\lambda+\lambda_{j}>0$ para todo $j=1, \ldots, n$. Portanto, no intervalo $\left(-\lambda_{1}, \infty\right)$ a função $\|p(\lambda)\|$ é contínua, decrescente e côncava. 
Vamos definir a função

$$
\phi_{1}(\lambda)=\|p(\lambda)\|-\Delta .
$$

Agora podemos dividir o problema em 4 casos:

(i) Quando $B$ é definida positiva e $\left\|B^{-1} q\right\| \leq \Delta$. Neste caso, como já vimos, a solução de (1.1) é dada por $p(0)=-B^{-1} g$;

(ii) Quando $B$ é definida positiva mas $\left\|B^{-1} q\right\|>\Delta$. Neste caso, se usarmos $\lambda=0$, temos $\left\|B^{-1} q\right\|>\Delta$. Então é preciso que se aumente o valor de $\lambda$ para que a norma de $p(\lambda)$ diminua (veja a equação (1.3)).

Como $-\lambda_{1}<0$ (porque $B$ é definida positiva), não precisamos nos preocupar com os pontos de descontinuidade de $\|p(\lambda)\|$, pois podemos procurar uma raiz $\lambda^{*}$ de $\phi_{1}(\lambda)$ no intervalo $(0, \infty)$.

Pelo Lema 1.1.2-(iii), a solução de (1.1) é dada por $p\left(\lambda^{*}\right)=-\left(B+\lambda^{*} I\right)^{-1} g$;

(iii) Quando $B$ não é definida positiva e $q_{1}^{T} g \neq 0$. Neste caso, valem os limites (1.4) e (1.5). Então sabemos que existe um valor no intervalo $\left(-\lambda_{1}, \infty\right)$ em que $\|p(\lambda)\|=\Delta$ (pois neste intervalo a função é contínua e assume valores no intervalo $(0, \infty)$, ao qual pertence $\Delta)$.

Como $B$ não é definida positiva, temos que $\lambda_{1} \leq 0$. Assim, afirmamos que, além de existir uma raiz $\lambda^{*}$ de $\phi_{1}(\lambda), \lambda^{*}>-\lambda_{1} \geq 0$.

Neste caso, a solução de (1.1) é dada por $p\left(\lambda^{*}\right)=-\left(B+\lambda^{*} I\right)^{-1} g$ (pelo Lema 1.1.2-(iii));

(iv) Quando $B$ não é definida positiva e $q_{1}^{T} g=0$. Este é o caso difícil. Neste caso não existe o limite (1.5) (veja o desenho de uma função $\|p(\lambda)\|$ para $q_{1}^{T} g=0$ na figura 1.2). Então não temos garantia de que a raiz de $\phi_{1}(\lambda)$ esteja no intervalo $\left(-\lambda_{1}, \infty\right)$.

Pelo Lema 1.1.1, temos que a solução de (1.1) é solução para uma equação do tipo (1.2) com $B+\lambda I$ semidefinida positiva.

Ora, para que $B+\lambda I$ seja semidefinida positiva, temos que $\lambda \in\left[-\lambda_{1}, \infty\right)$. Como não temos, necessariamente, a raiz $\lambda^{*}$ de $\phi_{1}(\lambda)$ no intervalo $\left(-\lambda_{1}, \infty\right)$, esta raiz pode estar exatamente em $-\lambda_{1}$. Neste caso, para qualquer $\lambda>-\lambda_{1},\|p(\lambda)\|<\Delta$.

Mas, com $\lambda^{*}=-\lambda_{1}$, temos que $B+\lambda^{*} I=B-\lambda_{1} I$, que é singular. Ou seja, existe um $z \neq 0$ tal que

$$
\left(B-\lambda_{1} I\right) z=0 \Rightarrow B z-\lambda_{1} z=0 \Rightarrow B z=\lambda_{1} z
$$

ou seja, $z$ é o autovetor de $B$ associado ao autovalor $\lambda_{1}$.

Podemos, sem perda de generalidade, usar $z=\tau \hat{z}$, para $\|\hat{z}\|=1$.

Note que, se $p$ satisfaz a equação (1.2) então $p+\tau \hat{z}$ também a satisfaz.

Note que, neste caso, podemos escrever 


$$
p=\sum_{j: \lambda_{j} \neq \lambda_{1}} \frac{q_{j}^{T} g}{\lambda_{j}-\lambda_{1}} q_{j}+\tau \hat{z},
$$

para todo $\tau \in \mathbb{R}$.

Assim,

$$
\begin{gathered}
\|p\|^{2}=\left(\sum_{j: \lambda_{j} \neq \lambda_{1}} \frac{q_{j}^{T} g}{\lambda_{j}-\lambda_{1}} q_{j}+\tau \hat{z}\right)^{T}\left(\sum_{j: \lambda_{j} \neq \lambda_{1}} \frac{q_{j}^{T} g}{\lambda_{j}-\lambda_{1}} q_{j}+\tau \hat{z}\right)= \\
\left\|\sum_{j: \lambda_{j} \neq \lambda_{1}} \frac{q_{j}^{T} g}{\lambda_{j}-\lambda_{1}} q_{j}\right\|^{2}+\left(\sum_{j: \lambda_{j} \neq \lambda_{1}} \frac{q_{j}^{T} g}{\lambda_{j}-\lambda_{1}} q_{j}\right)^{T}(\tau \hat{z})+\|\tau \hat{z}\|^{2} .
\end{gathered}
$$

Como $\hat{z}$ é ortogonal a $q_{j}$, temos que

$$
\left(\sum_{j: \lambda_{j} \neq \lambda_{1}} \frac{q_{j}^{T} g}{\lambda_{j}-\lambda_{1}} q_{j}\right)^{T}(\tau \hat{z})=0 .
$$

Ainda pela ortogonalidade de $Q$ e por $\|\hat{z}\|=1$, temos que

$$
\|p\|^{2}=\sum_{j: \lambda_{j} \neq \lambda_{1}} \frac{\left(q_{j}^{T} g\right)^{2}}{\left(\lambda_{j}-\lambda_{1}\right)^{2}}+\tau^{2},
$$

para todo $\tau \in \mathbb{R}$.

Desta forma, sempre é possível conseguir $\tau^{*}$ de tal forma que $\|p\|=\Delta$ para $\lambda=-\lambda_{1}$. Neste caso, o Lema 1.1.2-(iii) diz que a solução de (1.1) é $p$, ou seja, $p\left(-\lambda_{1}\right)+\tau^{*} \hat{z}$, para algum $\tau^{*}$.

\section{$1.2 \mathrm{O}$ algoritmo}

\subsubsection{Uma primeira versão do algoritmo}

Nesta seção, analisamos mais alguns resultados teóricos apresentados em [18]. Reproduzimos as provas de alguns lemas (no Apêndice), detalhamos outras e provamos algumas afirmações que não nos pareceram evidentes.

Agora que conhecemos bem a estrutura do problema, podemos pensar em algoritmos para resolvê-lo. 


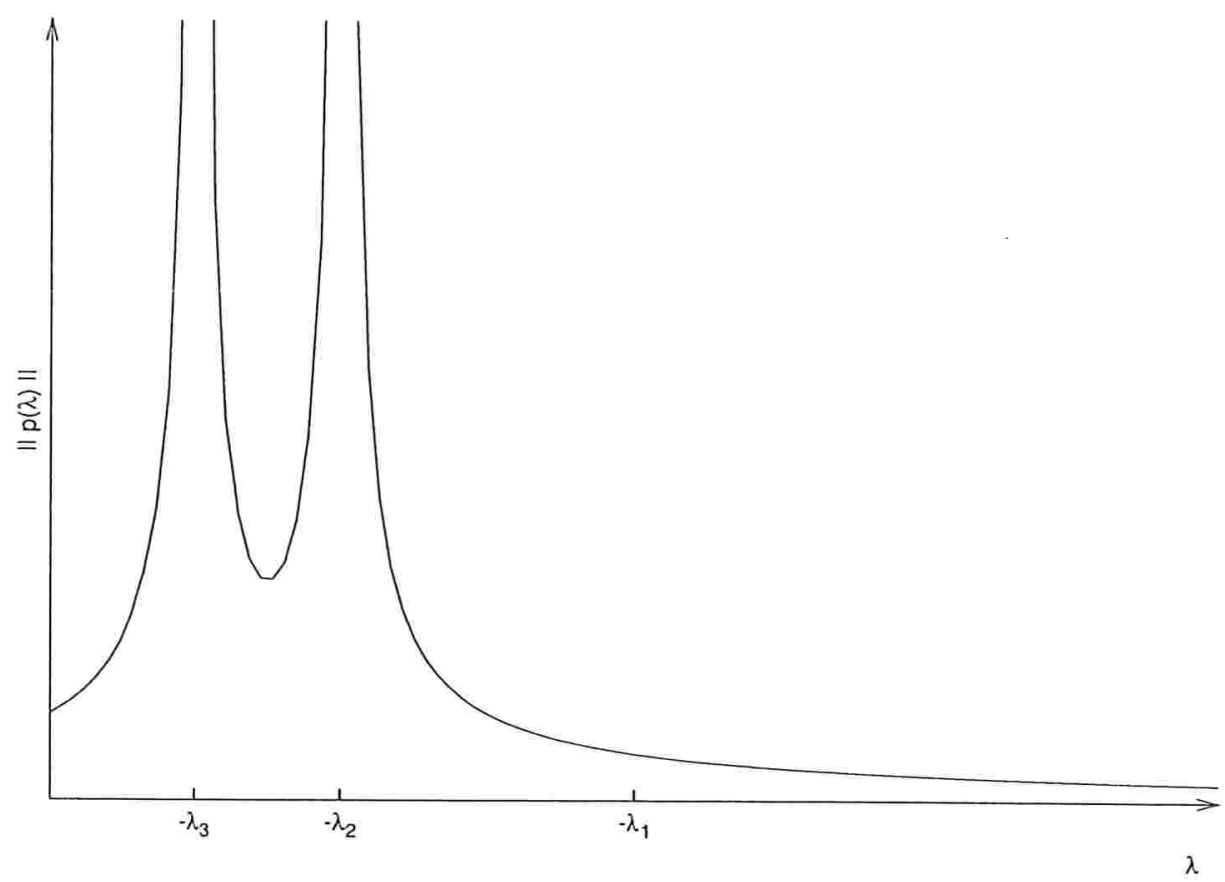

Figura 1.2: caso em que $q_{1}^{T} g=0$

$\mathrm{Na}$ maioria dos casos, o Método de Newton para encontrar raízes de uma função pode ser aplicado em $\phi_{1}(\lambda)$. No entanto, se definirmos

$$
\phi(\lambda)=\frac{1}{\Delta}-\frac{1}{\|p(\lambda)\|}
$$

podemos obter convergência melhor do Método, porque no intervalo $\left(-\lambda_{1}, \infty\right)$ a função $\phi(\lambda)$ é quase linear.

A cada passo do Método de Newton define-se

$$
\lambda^{k+1}=\lambda^{k}-\frac{\phi(\lambda)}{\phi^{\prime}(\lambda)}
$$

Lema 1.2.1 Seja $\phi(\lambda)=\frac{1}{\Delta}-\frac{1}{\|p(\lambda)\|}$, onde $p(\lambda)=-(B+\lambda I)^{-1} g$ com $B$ simétrica e $B+\lambda I$ definida positiva.

Então,

$$
-\frac{\phi(\lambda)}{\phi^{\prime}(\lambda)}=\left(\frac{\|p(\lambda)\|}{\|t(\lambda)\|}\right)^{2}\left(\frac{\|p(\lambda)\|-\Delta}{\Delta}\right),
$$

onde $t(\lambda)$ é a solução de $R^{T} t(\lambda)=p(\lambda)$ e $R$ é a matriz triangular superior tal que $B+\lambda I=$ $R^{T} R$. 
Prova : ver Apêndice.

Com base no Lema 1.1.2, podemos escrever o algoritmo de Newton:

Algoritmo 1 Dados $\Delta>0$ e $\lambda^{0} \geq 0 \operatorname{com} B+\lambda^{0} I$ definida positiva.

Passo 1. $k=0$

Passo 2. Fatore $B+\lambda^{k} I=R^{T} R$

Passo 3. Resolva $R^{T} R p_{k}=-g$ em $p_{k}$

Passo 4. Se o "critério de convergência foi satisfeito" então

Pare e devolva $p_{k}$ como solução

Passo 5. Resolva $R^{T} t_{k}=p_{k}$ em $t_{k}$

Passo 6. $\lambda^{k+1}=\lambda^{k}+\left(\frac{\left\|p_{k}\right\|}{\left\|t_{k}\right\|}\right)^{2}\left(\frac{\left\|p_{k}\right\|-\Delta}{\Delta}\right)$

Passo 7. $k=k+1$

Passo 8. volte para o Passo 2

É claro que é necessário salvaguardar $\lambda^{k}$ para que $B+\lambda^{k} I$ seja definida positiva a cada passo e para que tenhamos garantia de convergência do método.

Mas, antes de nos focar na salvaguarda de $\lambda^{k}$ e nos critérios de convergência do algoritmo, concentremo-nos no caso difícil. Note em especial que, quando temos $g=0$, o algoritmo está mal-definido.

Como visto anteriormente, para a resolução do caso difícil é necessário fornecer $z$ que é o autovetor associado ao autovalor $\lambda_{1}$. Como é computacionalmente caro calcular o autovetor, calcularemos uma aproximação deste.

\subsubsection{Cálculo da aproximação de $z$}

Vejamos o lema que segue:

Lema 1.2.2 (Lema 3.4 de [18]) Seja $0<\sigma<1$ dado e suponha que

$$
B+\lambda I=R^{T} R, \quad(B+\lambda I) p=-g, \quad \lambda \geq 0 .
$$

Seja $z \in \mathbb{R}^{n}$ tal que

$$
\|p+z\|=\Delta, \quad\|R z\|^{2} \leq \sigma\left(\|R p\|^{2}+\lambda \Delta^{2}\right) .
$$

Então

$$
-\varphi(p+z) \geq \frac{1}{2}(1-\sigma)\left(\|R p\|^{2}+\lambda \Delta^{2}\right) \geq(1-\sigma)\left|\varphi^{*}\right|
$$


onde $\varphi^{*}$ é o valor ótimo de (1.1).

Prova : ver Apêndice.

Veja que, como conseqüência do Lema 1.2.2, temos

$$
\begin{gathered}
-\varphi(p+z) \geq(1-\sigma)\left|\varphi^{*}\right| \Rightarrow-\varphi(p+z)-\left|\varphi^{*}\right| \geq-\sigma\left|\varphi^{*}\right| \Rightarrow \\
\sigma\left|\varphi^{*}\right| \geq \varphi(p+z)+\left|\varphi^{*}\right| \geq \varphi(p+z)-\varphi^{*} .
\end{gathered}
$$

Como $\varphi(p+z)-\varphi^{*} \geq 0$, temos que

$$
\left|\varphi(p+z)-\varphi^{*}\right| \leq \sigma\left|\varphi^{*}\right|
$$

Ou seja, se vale (1.6), $p+z$ é uma solução próxima da solução ótima de (1.1). Mais ainda, quanto mais próximo de 0 está $\|R \hat{z}\|$, mais próximo $p+z$ está da solução ótima de (1.1).

O Lema 1.2.2 é conveniente no caso difícil, quando vale que $B+\lambda I=R^{T} R$ (definida positiva), $(B+\lambda I) p=-g, \lambda \geq 0$ e $\|p\|<\Delta$. Neste caso temos $\lambda$ próximo de $-\lambda_{1}$.

Então estamos interessados num algoritmo que calcule um vetor $\hat{z}$, com $\|\hat{z}\|=1$, de tal forma que, quando $\lambda$ se aproxima de $-\lambda_{1},\|R \hat{z}\|$ se aproxima de 0 . Calculado $\hat{z}$, podemos calcular $\tau$ de maneira que $\|p+\tau \hat{z}\|=\Delta$.

Note que a solução ótima de (1.1) é dada por $p+z$, com $\left(B-\lambda_{1}\right) p=-g$ e $z$ o autovetor de $B$ associado a $\lambda_{1}$. Com $\hat{z}$ calculado como dito acima, temos que $\tau \hat{z}$ se aproxima do autovetor $z$.

Uma maneira de calcular $\hat{z}$ é usando uma técnica introduzida em [7] para estimar o menor valor singular de uma matriz triangular $R$. O Lema 1.2.3 mostra que esta técnica satisfaz os requisitos apresentados acima. Nesta técnica, um vetor $e$ com componentes 1 ou -1 é escolhido de maneira que a solução $w$ do sistema $R^{T} w=e$ seja grande. A idéia é escolher o sinal das componentes de $e$ de maneira a obter máximo crescimento local de $w$ durante a resolução do sistema. Depois, resolve-se o sistema $R v=w$ para $v$. O vetor $v$ é tal que, se $\hat{z}$ for definido como $\hat{z}=\frac{v}{\|v\|}$, então $\|R \hat{z}\|$ se aproxima do menor valor singular de $R$. Essa técnica é atrativa porque não é computacionalmente cara (gasta em torno de $n^{2}$ operações aritméticas) e, por isso, a implementamos.

Lema 1.2.3 Seja $(B+\lambda I)=R^{T} R$ uma matriz definida positiva. Sejam $v \in \mathbb{R}^{n}$ o vetor calculado utilizando a técnica descrita em [7] e $\hat{z}=\frac{v}{\|v\|}$. Então $\|R \hat{z}\|$ se aproxima de 0 quando $\lambda$ se aproxima de $-\lambda_{1}$, com $\lambda_{1}$ o menor autovalor de $B$. 
Prova : ver Apêndice.

Dado que estamos interessados no caso em que $\lambda$ se aproxima de $-\lambda_{1}$, a matriz $R$ é quase singular e isso poderia levar a dificuldades numéricas. O critério de convergência (1.8) (apresentado na seção 1.2.4) garante que o algoritmo pára antes que dificuldades numéricas apareçam. saber

Calculado $\hat{z}$, temos 2 possíveis escolhas para o valor de $\tau$ que satisfazem $\|p+\tau \hat{z}\|=\Delta$, a

$$
\begin{gathered}
\|p+\tau \hat{z}\|=\Delta \Rightarrow\|p+\tau \hat{z}\|^{2}=\Delta^{2} \Rightarrow\|p+\tau \hat{z}\|^{2}-\Delta^{2}=0 \Rightarrow \\
\|p\|^{2}+2 \tau\left(p^{T} \hat{z}\right)+\tau^{2}\|\hat{z}\|^{2}-\Delta^{2}=0 .
\end{gathered}
$$

Como $\|\hat{z}\|=1$, temos que

$$
\begin{gathered}
\tau^{2}+2 \tau\left(p^{T} \hat{z}\right)+\|p\|^{2}-\Delta^{2}=0 \Rightarrow \\
\tau=\frac{-2\left(p^{T} \hat{z}\right) \pm \sqrt{4\left(\left(p^{T} \hat{z}\right)^{2}-\left(\|p\|^{2}-\Delta^{2}\right)\right)}}{2} \Rightarrow \\
\tau=-p^{T} \hat{z} \pm \sqrt{\left(p^{T} \hat{z}\right)^{2}-\left(\|p\|^{2}-\Delta^{2}\right)} .
\end{gathered}
$$

Pela equação (A.10), a escolha de $\tau$ com menor magnitude fornece um valor menor de $\varphi$.

Esta escolha, portanto, é

$$
\tau=-p^{T} \hat{z}+\operatorname{sgn}\left(p^{T} \hat{z}\right) \sqrt{\left(\left(p^{T} \hat{z}\right)^{2}-\left(\|p\|^{2}-\Delta^{2}\right)\right)} .
$$

\subsubsection{Salvaguarda de $\lambda^{k}$}

Agora que temos todas as ferramentas para calcular $\hat{z}$ e $\tau$, vamos voltar ao problema da salvaguarda de $\lambda^{k}$.

A salvaguarda de $\lambda^{k}$ depende do fato de que $\phi(\lambda)$ é convexa e estritamente decrescente no intervalo $\left(-\lambda_{1}, \infty\right)$. Isso nos mostra que o Método de Newton, quando aplicado à função $\phi$, partindo de $\lambda^{k} \in\left(-\lambda_{1}, \infty\right)$ e $\phi\left(\lambda^{k}\right)>0$ gera uma seqüência monotonicamente crescente que converge para a solução $\phi\left(\lambda^{*}\right)=0$. Além disso, se $\lambda^{k} \in\left(-\lambda_{1}, \infty\right)$ e $\phi\left(\lambda^{k}\right)<0$, temos que ou 
$\lambda^{k+1} \leq-\lambda_{1}$ ou $\phi\left(\lambda^{k+1}\right)>0$.

Está claro que devemos manter os valores de $\lambda^{k}$ no intervalo $\left(-\lambda_{1}, \infty\right)$, que é justamente onde está a solução $\lambda^{*}$.

Manteremos, então, 3 parâmetros: $\lambda_{L}, \lambda_{U}$ e $\lambda_{S}$. O intervalo $\left[\lambda_{L}, \lambda_{U}\right]$ conterá a solução $\lambda^{*}$ e será reduzido a cada iteração. $\lambda_{S}$ será um limitante inferior para $-\lambda_{1}$.

Para a salvaguarda de $\lambda^{k}$ temos os seguintes passos:

Passo 1. $\lambda^{k}=\max \left\{\lambda^{k}, \lambda_{L}\right\}$

Passo 2. $\lambda^{k}=\min \left\{\lambda^{k}, \lambda_{U}\right\}$

Passo 3. Se $\lambda^{k} \leq \lambda_{S}$ então $\lambda^{k}=\max \left\{0.001 \lambda_{U}, \sqrt{\lambda_{L} \lambda_{U}}\right\}$

Claramente os Passos 1 e 2 colocam $\lambda^{k}$ no intervalo $\left[\lambda_{L}, \lambda_{U}\right]$. O Passo 3 permite que se reduza o tamanho do intervalo de incerteza. Este tipo de atualização foi já usado em outras ocasiões (veja [11]) e os resultados foram satisfatórios. Note que o tamanho do intervalo só é reduzido quando $\lambda_{S} \in\left[\lambda_{L}, \lambda_{U}\right]$. Se o tamanho do intervalo de incerteza fica longe de 0 , o Passo 3 pode ser executado apenas um número finito de vezes. Isso ficará claro com as regras de atualização de $\lambda_{S}, \lambda_{L}$ e $\lambda_{U}$. No final deste capítulo veremos a importância deste fato.

Veja agora como obter valores iniciais para $\lambda_{L}, \lambda_{U}$ e $\lambda_{S}$ e como atualizá-los.

\subsubsection{Valores iniciais de $\lambda_{L}, \lambda_{U}$ e $\lambda_{S}$}

Encontrar $\lambda_{1}$ o menor autovalor da matriz simétrica $B$ equivale a encontrar o mínimo de

$$
\frac{x^{T} B x}{x^{T} x}
$$

onde $x \in \mathbb{R}^{n}$ não é nulo (veja, por exemplo, [12]).

Note que, se escolhermos $x$ como o vetor canônico $e_{i}$ (componente $i$ é igual a 1 e as restantes iguais a 0 ), temos que

$$
\frac{e_{i}^{T} B e_{i}}{e_{i}^{T} e_{i}}=b_{i i} \geq \lambda_{1}, \text { para } i=1, \ldots, n,
$$

onde $b_{i i}$ é o i-ésimo elemento da diagonal de $B$.

Assim,

$$
\min _{1 \leq i \leq n}\left\{b_{i i}\right\} \geq \lambda_{1} \Rightarrow-\min _{1 \leq i \leq n}\left\{b_{i i}\right\} \leq-\lambda_{1} \Rightarrow \max _{1 \leq i \leq n}\left\{-b_{i i}\right\} \leq-\lambda_{1}
$$

Portanto, podemos começar o algoritmo com 


$$
\lambda_{S}=\max _{1 \leq i \leq n}\left\{-b_{i i}\right\}
$$

Para escolhermos um valor inicial para $\lambda_{L}$ e $\lambda_{U}$, veja o seguinte lema:

Lema 1.2.4 Tome $B, g$ e $\Delta$ do problema (1.1).

Um limitante inferior $\lambda_{L}$ para $\lambda^{*}$ solução de $\phi(\lambda)$ é

$$
\lambda_{L}=\frac{\|g\|}{\Delta}-\|B\|_{1}
$$

e um limitante superior $\lambda_{U}$ é

$$
\lambda_{U}=\frac{\|g\|}{\Delta}+\|B\|_{1}
$$

Prova : ver Apêndice.

Então já temos uma maneira de escolher os valores iniciais de $\lambda_{U}$ e $\lambda_{S}$. Lembre-se que, como visto na seção anterior, a solução $\lambda^{*}$ que procuramos, além de estar no intervalo $\left(-\lambda_{1}, \infty\right)$ é maior ou igual a 0 .

Portanto, como valor inicial de $\lambda_{L}$ temos

$$
\lambda_{L}=\max \left\{0, \lambda_{S}, \frac{\|g\|}{\Delta}-\|B\|_{1}\right\}
$$

\subsubsection{Atualização dos valores de $\lambda_{L}, \lambda_{U}$ e $\lambda_{S}$}

Vejamos primeiro como atualizar o valor de $\lambda_{S}$.

Note que, quando $\lambda^{k} \in\left(-\lambda_{1}, \infty\right)$ e $\phi\left(\lambda^{k}\right)<0$, temos $\|p\|<\Delta$, e podemos calcular $\hat{z}$ e $\tau$ como apresentado na seção 1.2.2.

Veja agora o seguinte lema:

Lema 1.2.5 Seja $B+\lambda I$ uma matriz definida positiva tal que $B+\lambda I=R^{T} R$. Para qualquer $\hat{z}$ tal que $\|\hat{z}\|=1$ vale que

$$
\lambda-\|R \hat{z}\|^{2} \leq-\lambda_{1}
$$


Prova : ver Apêndice.

Então, neste caso, podemos tomar $\lambda_{S}$ como

$$
\lambda_{S}=\max \left\{\lambda_{S}, \lambda^{k}-\|R \hat{z}\|^{2}\right\}
$$

já que $\lambda_{S}$ também era um limitante inferior para $-\lambda_{1}$.

No caso em que $\lambda^{k} \leq-\lambda_{1}$ temos $B+\lambda^{k} I$ indefinida. Desta forma, não é possível fazer a decomposição de Cholesky.

Porém, note que, durante a tentativa de decomposiçao, pode-se calcular $\delta \geq 0$ de forma que

$$
B+\lambda^{k} I+\delta e_{l} e_{l}^{T}
$$

seja singular e $l$ é exatamente a posição da matriz $B+\lambda^{k} I$ onde a decomposição falha.

Ainda é possível determinar o vetor $u \in \mathbb{R}^{n}$ tal que

$$
\left(B+\lambda^{k} I+\delta e_{l} e_{l}^{T}\right) u=0,
$$

com $u_{l}=1$ e $u_{i}=0$ para todo $i>l$.

Agora veja o lema a seguir:

Lema 1.2.6 Seja $\delta \geq 0$ tal que $B+\lambda I+\delta e_{l} e_{l}^{T}$ é singular, com el a l-ésima coluna da matriz identidade. Seja $u \in \mathbb{R}^{n}$ tal que $\left(B+\lambda I+\delta e_{l} e_{l}^{T}\right) u=0$, com $u_{l}=1$ e $u_{i}=0$ para todo $i>l$.

Então

$$
\lambda+\frac{\delta}{\|u\|^{2}} \leq-\lambda_{1}
$$

Prova : ver Apêndice.

Então, neste caso, podemos tomar $\lambda_{S}$ como sendo

$$
\lambda_{S}=\max \left\{\lambda_{S}, \lambda^{k}+\frac{\delta}{\|u\|^{2}}\right\} .
$$

A atualização de $\lambda_{L}$ e $\lambda_{U}$ é a esperada. Desta maneira, para atualizar os valores de $\lambda_{L}$, $\lambda_{U}$ e $\lambda_{S}$ basta seguir os seguintes passos:

Passo 1. Se $\lambda^{k} \in\left(-\lambda_{1}, \infty\right)$ e $\phi\left(\lambda^{k}\right)<0$ então

$$
\begin{aligned}
& \lambda_{U}=\min \left\{\lambda_{U}, \lambda^{k}\right\} \\
& \lambda_{S}=\max \left\{\lambda_{S}, \lambda^{k}-\|R \hat{z}\|^{2}\right\}
\end{aligned}
$$

Senão 


$$
\lambda_{L}=\max \left\{\lambda_{L}, \lambda^{k}\right\}
$$

Passo 2. Se $\lambda^{k} \leq-\lambda_{1}$ então

$$
\begin{aligned}
& \text { compute } \delta \text { e }\|u\|^{2} \\
& \lambda_{S}=\max \left\{\lambda_{S}, \lambda^{k}+\frac{\delta}{\|u\|^{2}}\right\}
\end{aligned}
$$

Passo 3. $\lambda_{L}=\max \left\{\lambda_{L}, \lambda_{S}\right\}$

\subsubsection{Critérios de convergência}

Agora, para que o algoritmo fique completo, basta apenas definir os critérios de convergência. Queremos que ele pare com uma solução próxima do ótimo do problema (1.1).

Dados $\sigma_{1}, \sigma_{2} \in(0,1)$ e $\lambda^{k} \geq 0$ tal que $B+\lambda^{k} I$ é definida positiva, calcula-se $p$ usando o Método de Newton (Algoritmo 1). Se

$$
|\Delta-\|p\|| \leq \sigma_{1} \Delta, \text { ou }\|p\| \leq \Delta, \lambda^{k}=0
$$

então o algoritmo pára com $s=p$ como solução do problema (1.1).

No caso difícil, sempre que $\|p\|<\Delta$ (ou seja, sempre que $\phi\left(\lambda^{k}\right)<0$ ), $\hat{z}$ é calculado. Neste caso, quando

$$
\|R(\tau \hat{z})\|^{2} \leq \sigma_{1}\left(2-\sigma_{1}\right) \max \left\{\sigma_{2},\|R p\|^{2}+\lambda^{k} \Delta^{2}\right\}
$$

o algoritmo pára com $s=p+\tau \hat{z}$ como solução do problema (1.1).

Quando tanto (1.7) como (1.8) são satisfeitos, devemos escolher entre $p$ e $p+\tau \hat{z}$ o que fornece menor valor de $\varphi$.

Isso é fácil de verificar. Olhando para a equação (A.10) (da prova do Lema 1.2.2), é fácil ver que

$$
\varphi(p+\tau \hat{z}) \leq \varphi(p) \Leftrightarrow\|R(\tau \hat{z})\|^{2} \leq \lambda^{k}\left(\Delta^{2}-\|p\|^{2}\right) .
$$

Veremos agora que, quando o algoritmo pára, se o critério (1.7) ou (1.8) for satisfeito, o algoritmo de fato termina com uma solução $s$ próxima do ótimo para o problema (1.1), com $s$ tal que

$$
\varphi(s)-\varphi^{*} \leq \sigma_{1}\left(2-\sigma_{1}\right) \max \left\{\left|\varphi^{*}\right|, \sigma_{2}\right\}, \quad\|s\| \leq\left(1+\sigma_{1}\right) \Delta .
$$

Primeiro analisemos o caso em que o critério (1.7) é satisfeito. 
Lema 1.2.7 (Lema 3.13 de [18]) Seja $0<\sigma<1$ dado e suponha que

$$
B+\lambda I=R^{T} R, \quad(B+\lambda I) p=-g, \quad \lambda \geq 0 .
$$

Se $\|p\| \geq(1-\sigma) \Delta$ então

$$
-\varphi(p) \geq \frac{1}{2}(1-\sigma)^{2}\left(\|R p\|^{2}+\lambda \Delta^{2}\right) \geq(1-\sigma)^{2}\left|\varphi^{*}\right|,
$$

onde $\varphi^{*}$ é o valor ótimo de (1.1).

Prova : ver Apêndice.

Daí segue que, se o critério (1.7) for satisfeito então a solução fornecida pelo algoritmo satisfaz (1.9) (para verificar basta fazer algumas manipulações na inequação que resulta do Lema 1.2.7).

Agora vejamos o caso em que o (1.8) é satisfeito.

Se $\|R p\|^{2}+\lambda^{k} \Delta>\sigma_{2}$ então as hipóteses do Lema 1.2 .2 valem para $\sigma=\sigma_{1}\left(2-\sigma_{1}\right)$ e, portanto, (1.9) vale para $s=p+\tau \hat{z}$ (basta fazer as mesmas manipulações da inequação que resulta do Lema 1.2.2 que foram feitas para verificar o critério anterior).

Suponha agora que $\|R p\|^{2}+\lambda^{k} \Delta \leq \sigma_{2}$. Se $\varphi^{*}=\varphi\left(p+z^{*}\right)$ com $\left\|p+z^{*}\right\| \leq \Delta$ então a equação (A.10) (da prova do Lema 1.2.2) nos diz que

$$
\left|\varphi^{*}\right|=-\varphi^{*} \leq \frac{1}{2}\left(\|R p\|^{2}+\lambda^{k} \Delta^{2}\right) \leq \frac{1}{2} \sigma_{2}
$$

Usando novamente (A.10), temos que

$$
\varphi(p+\tau \hat{z})=-\frac{1}{2}\left(\|R p\|^{2}+\lambda^{k} \Delta^{2}\right)+\frac{1}{2}\|R(\tau \hat{z})\|^{2} \leq \varphi^{*}+\frac{1}{2} \sigma(2-\sigma 1) \sigma_{2} .
$$

Ou seja, quando o critério (1.8) é satisfeito, também vale (1.9).

\subsubsection{Versão final do algoritmo}

Agora que já dispomos de todos os elementos para a construção de um algoritmo para chegar à solução do problema (1.1), vamos escrevê-lo por completo.

Algoritmo 2 Dados uma matriz simétrica $B$, um vetor $g, \Delta>0, \lambda^{0} \geq 0, \sigma_{1}, \sigma_{2} \in(0,1)$

Passo 1. Valores iniciais de $\lambda_{L}, \lambda_{U}, \lambda_{S}$ e $k$

$$
\lambda_{S}=\max _{1 \leq i \leq n}\left\{-b_{i i}\right\}
$$




$$
\begin{aligned}
& \lambda_{L}=\max \left\{0, \lambda_{S}, \frac{\|g\|}{\Delta}-\|B\|_{1}\right\} . \\
& \lambda_{U}=\frac{\|g\|}{\Delta}+\|B\|_{1} \\
& k=0
\end{aligned}
$$

Passo 2. Salvaguarda de $\lambda^{k}$

$$
\begin{aligned}
& \lambda^{k}=\max \left\{\lambda^{k}, \lambda_{L}\right\} \\
& \lambda^{k}=\min \left\{\lambda^{k}, \lambda_{U}\right\} \\
& \text { Se } \lambda^{k} \leq \lambda_{S} \text { então } \lambda^{k}=\max \left\{0.001 \lambda_{U}, \sqrt{\lambda_{L} \lambda_{U}}\right\}
\end{aligned}
$$

Passo 3. Tentativa de fatoração de $B+\lambda^{k} I$

Use Cholesky para fatorar $B+\lambda^{k} I=R^{T} R$

Se a fatoração não foi possível então

$$
\begin{aligned}
& \text { compute } \delta \text { e }\|u\|^{2} \text { (como descrito na seção 1.2.3.2) } \\
& \lambda_{S}=\max \left\{\lambda_{S}, \lambda^{k}+\frac{\delta}{\|u\|^{2}}\right\} \\
& \text { vá para o Passo } 6
\end{aligned}
$$

Passo 4. Resolva $R^{T} R p_{k}=--g$ em $p_{k}$

Passo 5. Cálculo da aproximação de z

Se $\left\|p_{k}\right\|<\Delta$ então calcule $\hat{z}$ usando a técnica de [7]

$$
\tau=-p_{k}^{T} \hat{z}+\operatorname{sgn}\left(p_{k}^{T} \hat{z}\right) \sqrt{\left(\left(p_{k}^{T} \hat{z}\right)^{2}-\left(\left\|p_{k}\right\|^{2}-\Delta^{2}\right)\right)}
$$

Passo 6. Atualização dos valores de $\lambda_{L}, \lambda_{U}, \lambda_{S}$

Se $\lambda^{k} \in\left(-\lambda_{1}, \infty\right)$ e $\phi\left(\lambda^{k}\right)<0$ então

$$
\begin{aligned}
& \lambda_{U}=\min \left\{\lambda_{U}, \lambda^{k}\right\} \\
& \lambda_{S}=\max \left\{\lambda_{S}, \lambda^{k}-\|R \hat{z}\|^{2}\right\}
\end{aligned}
$$

Senão

$$
\begin{aligned}
& \lambda_{L}=\max \left\{\lambda_{L}, \lambda^{k}\right\} \\
& \lambda_{L}=\max \left\{\lambda_{L}, \lambda_{S}\right\}
\end{aligned}
$$

Passo 7. Critérios de convergência

Passo 7.1 Se $\left\|p_{k}\right\| \leq \Delta$ e $\lambda^{k}=0$ então 
Pare e devolva $p_{k}$ como solução

Passo 7.2 Teste se $\left|\Delta-\left\|p_{k}\right\|\right| \leq \sigma_{1} \Delta$

Passo 7.3 Teste se $\|R(\tau \hat{z})\|^{2} \leq \sigma_{1}\left(2-\sigma_{1}\right) \max \left\{\sigma_{2},\left\|R p_{k}\right\|^{2}+\lambda^{k} \Delta^{2}\right\}$

Passo 7.4 Decisão do critério a ser aceito

Se apenas o Passo 7.2 foi satisfeito então

Pare e devolva $p_{k}$ como solução

Se apenas o Passo 7.3 foi satisfeito então

Pare e devolva $p_{k}+\tau \hat{z}$ como solução

Se tanto o Passo 7.2 como o Passo 7.3 foram satisfeitos então

Se $\|R(\tau \hat{z})\|^{2} \leq \lambda^{k}\left(\Delta^{2}-\left\|p_{k}\right\|^{2}\right)$ então

Pare e devolva $p_{k}+\tau \hat{z}$ como solução

Senão

Pare e devolva $p_{k}$ como solução

Passo 8. Atualização de $\lambda^{k}$ e $k$

Se foi possível a fatoração de Cholesky e $g \neq 0$ então

Resolva $R^{T} t_{k}=p_{k}$ em $t_{k}$

$$
\lambda^{k+1}=\lambda^{k}+\left(\frac{\left\|p_{k}\right\|}{\left\|t_{k}\right\|}\right)^{2}\left(\frac{\left\|p_{k}\right\|-\Delta}{\Delta}\right)
$$

Senão

$$
\lambda^{k+1}=\lambda_{S}
$$

$k=k+1$

\section{Volte para o Passo 2}

Depois de um número finito de iterações o Algoritmo 2 pára com $\lambda^{k} \in\left(-\lambda_{1}, \infty\right)$ e $\phi\left(\lambda^{k}\right) \geq 0$ ou com um intervalo de incerteza arbitrariamente pequeno. Vejamos o porquê.

O primeiro ponto a ser observado é que a terceira atribuição do Passo 2 faz com que o intervalo de incerteza seja reduzido quando $\lambda_{S} \in\left[\lambda_{L}, \lambda_{U}\right]$. Quando este passo é executado, $\lambda^{k}$ é "jogado" dentro do intervalo aberto $\left(\lambda_{L}, \lambda_{U}\right.$ ) (quando $\lambda_{L} \neq \lambda_{U}$ ). No Passo 6, com a atualização dos valores de $\lambda_{L}$ e $\lambda_{U}$, temos o intervalo de incerteza reduzido. Como $\lambda_{S}$ é um limitante inferior para $-\lambda_{1}$, essa diminuição do tamanho do intervalo só pode acontecer enquanto $-\lambda_{1}$ ainda for candidato à raiz de $\phi(\lambda)$.

Se $-\lambda_{1}$ é a raiz de $\phi(\lambda)$, o intervalo será reduzido até que fique arbitrariamente pequeno, contendo $-\lambda_{1}$. Neste caso, $\lambda^{k}$ ficará próximo de $-\lambda_{1}$ e, portanto, $R$ será quase singular, 
fazendo com que (1.8) seja satisfeito e o Algoritmo 2 pare.

Quando a raiz de $\phi(\lambda)$ não está em $-\lambda_{1}$, teremos, em um número finito de iterações, $-\lambda_{1}<\lambda_{L}$. Ou seja, $\lambda_{S}$ sairá do intervalo de incerteza e nunca mais voltará. Neste ponto, teremos $\lambda^{k} \in\left(-\lambda_{1}, \infty\right)$. Se $\phi\left(\lambda^{k}\right)<0$, o Método de Newton garante que teremos $\phi\left(\lambda^{k}\right) \geq 0$. Ou seja, (1.7) será satisfeito e o Algoritmo 2 pára.

\subsection{Resultados numéricos}

Implementamos o Algoritmo 2 numa subrotina de FORTRAN 77, chamada MEQB. Para que esta subrotina seja testada, é preciso construir uma instância do problema (1.1). Para isso, é preciso fornecer a matriz simétrica $B$, o vetor $g$ e o escalar $\Delta$. Além disso, também é necessário que se estabeleça o valor inicial de $\lambda$.

Em [18] é sugerida uma maneira para se construir a matriz $B$, o vetor $g$ e o valor inicial de $\lambda$ de forma sistemática e conveniente. Utilizamos estes testes para verificar se a subrotina MEQB apresentava o mesmo comportamento da implementação mencionada em [18], o que nos levaria a acreditar que nossa implementação de fato estava correta. Explicamos a construção das instâncias para testes abaixo.

Para o valor inicial de $\lambda$, uma escolha razoável é

$$
\lambda=\frac{\|g\|}{\Delta} .
$$

Uma maneira para se construir a matriz $B$ é, para alguma matriz ortogonal $Q$ e uma matriz diagonal $D$, calcular $B=Q D Q^{T}$. Assim, calculando $g=Q \hat{g}$, para algum $\hat{g}$, podemos gerar uma instância potencialmente pertencente ao caso difícil preenchendo com 0 a componente de $\hat{g}$ que corresponde ao menor elemento de $D$.

Obtemos a matriz ortogonal $Q$ de forma que $Q=Q_{1} Q_{2} Q_{3}$, onde

$$
Q_{j}=I-2 \frac{w_{j} w_{j}^{T}}{\left\|w_{j}\right\|^{2}}, j=1,2,3
$$

onde as componentes de $w_{j}$ são uniformemente distribuídas entre $(-1,1)$.

Para se obter valores pseudo-aleatórios, uniformemente distribuídos, usou-se a função RAND apresentada em [21]. Dada uma semente, esta função gera um número pseudo-aleatório no intervalo $(0,1)$ e muda o valor da semente. Assim, sucessivas chamadas à função RAND geram números pseudo-aleatórios, que podem ser reproduzidos se a semente usada inicialmente for mantida.

Agora, basta especificar $D, \hat{g}$ e $\Delta$. A escolha desses parâmetros levam a diferentes tipos de problemas. 
Em [18] são sugeridos 4 tipos de problemas: o caso geral, o caso difícil, o caso definido positivo e o caso de ponto de sela.

Para a obtenção de qualquer um dos tipos de problemas, tanto $D$ como $\hat{g}$ têm, inicialmente, suas componentes uniformemente distribuídas entre $(-1,1)$. Isso basta para se gerar uma instância do caso geral. Para se obter uma instância do caso difícil, atribui-se 0 à componente de $\hat{g}$ que correspondente à menor componente de $D$. Para o caso definido positivo, basta trocar $D=\operatorname{diag}\left(d_{1}, d_{2}, \ldots, d_{n}\right)$ por $\bar{D}=\operatorname{diag}\left(\left|d_{1}\right|,\left|d_{2}\right|, \ldots,\left|d_{n}\right|\right)$. Para o caso de ponto de sela, é atribuído 0 a todas as componentes de $\hat{g}$.

Para valores de $\Delta$ no intervalo $(0,1)$, a escolha sugerida para o $\lambda$ inicial é muito conveniente, deixando o teste mais fácil. Por isso, foram escolhidos, para os testes, valores de $\Delta$ uniformemente distribuídos em $(0,100)$.

Nas tabelas 1.1, 1.2, 1.3 e 1.4 são apresentados os dados obtidos na execução dos testes para cada um dos casos. Em todos eles foram testadas instâncias de dimensão 10, 20, 40, 60, 80 e 100. Para cada um dos tamanhos foram usados, como valores de $\sigma_{1}$ e $\sigma_{2}$ (sempre utilizando $\sigma_{1}=\sigma_{2}$ ) $, 10^{-1}, 10^{-3}$ e $10^{-5}$. E, por fim, para cada um dos problemas obtidos (ou seja, dado uma das seis dimensões citadas e um dos três valores de $\sigma_{1}$ e $\sigma_{2}$ ), foram geradas 50 instâncias diferentes, com um valor de $\Delta$ no intervalo $(0,100)$ para cada uma.

Em [18] não fica claro quais os valores de $\sigma_{1}$ e $\sigma_{2}$ utilizados para a obtenção dos resultados lá apresentados. Por isso testamos o comportamento de MEQB usando os três valores de $\sigma_{1}$ e $\sigma_{2}$ apresentados acima. Os resultados obtidos pelo MEQB para $\sigma_{1}=\sigma_{2}=10^{-1}$ são equivalentes aos apresentados em [18], o que nos faz crer que este seja o valor de $\sigma_{1}$ utilizado em [18] e nos indica que a nossa implementação está correta.

Para a compreensão das tabelas apresentadas, lembre-se de que no Algoritmo 2 existem três critérios de convergência que podem ser satisfeitos, a saber:

- critério 1: $\|p\| \leq \Delta \mathrm{e} \lambda^{k}=0$

- critério 2: $|\Delta-\|p\|| \leq \sigma_{1} \Delta$

- critério 3: $\|R(\tau \hat{z})\|^{2} \leq \sigma_{1}\left(2-\sigma_{1}\right) \max \left\{\sigma_{2},\left\|R p_{k}\right\|^{2}+\lambda^{k} \Delta^{2}\right\}$ 
Tabela 1.1: Comportamento de MEQB para o caso geral

\begin{tabular}{|c|c|c|c|c|c|c|}
\hline \multirow{3}{*}{$\sigma_{1}=\sigma_{2}$} & \multicolumn{2}{|c|}{ número de iterações } & \multicolumn{3}{|c|}{ número de vezes que é satisfeito } \\
\cline { 2 - 7 } & dimensão & média & máximo & critério 1 & critério 2 & critério 3 \\
\hline \hline \multirow{4}{*}{$10^{-1}$} & 10 & 2.30 & 4 & 1 & 3 & 46 \\
\cline { 2 - 7 } & 20 & 2.32 & 5 & 0 & 4 & 46 \\
\cline { 2 - 7 } & 40 & 3.14 & 5 & 0 & 4 & 46 \\
\cline { 2 - 7 } & 60 & 3.20 & 5 & 0 & 5 & 45 \\
\cline { 2 - 7 } & 80 & 3.32 & 5 & 0 & 5 & 45 \\
\cline { 2 - 7 } & 100 & 3.42 & 5 & 0 & 8 & 42 \\
\hline \hline \multirow{5}{*}{$10^{-3}$} & 10 & 5.46 & 11 & 0 & 49 & 1 \\
\cline { 2 - 7 } & 20 & 6.12 & 11 & 0 & 44 & 6 \\
\cline { 2 - 7 } & 40 & 6.52 & 11 & 0 & 45 & 5 \\
\cline { 2 - 7 } & 60 & 7.10 & 11 & 0 & 47 & 3 \\
\cline { 2 - 7 } & 10 & 7.86 & 14 & 0 & 46 & 4 \\
\hline \hline \multirow{5}{*}{$10^{-5}$} & 100 & 7.20 & 12 & 0 & 45 & 5 \\
\cline { 2 - 7 } & 10 & 6.32 & 14 & 0 & 50 & 0 \\
\cline { 2 - 7 } & 20 & 6.70 & 13 & 0 & 50 & 0 \\
\cline { 2 - 7 } & 40 & 7.78 & 12 & 0 & 50 & 0 \\
\cline { 2 - 7 } & 60 & 7.78 & 14 & 0 & 50 & 0 \\
\cline { 2 - 7 } & 10 & 8.40 & 15 & 0 & & 3 \\
\hline
\end{tabular}

Tabela 1.2: Comportamento de MEQB para o caso difícil

\begin{tabular}{|c|c|c|c|c|c|c|}
\hline \multirow{3}{*}{$\sigma_{1}=\sigma_{2}$} & dimensão & \multicolumn{2}{|c|}{ número de iterações } & \multicolumn{3}{|c|}{ número de vezes que é satisfeito } \\
\cline { 2 - 7 } & média & máximo & critério 1 & critério 2 & critério 3 \\
\hline \hline \multirow{4}{*}{$10^{-1}$} & 10 & 2.44 & 5 & 1 & 0 & 49 \\
\cline { 2 - 7 } & 20 & 2.18 & 4 & 0 & 4 & 46 \\
\cline { 2 - 7 } & 40 & 2.90 & 4 & 0 & 3 & 47 \\
\cline { 2 - 7 } & 60 & 3.06 & 4 & 0 & 4 & 46 \\
\cline { 2 - 7 } & 80 & 3.22 & 5 & 0 & 5 & 45 \\
\cline { 2 - 7 } & 100 & 3.38 & 5 & 0 & 8 & 42 \\
\hline \hline \multirow{5}{*}{$10^{-3}$} & 10 & 7.12 & 10 & 0 & 9 & 41 \\
\cline { 2 - 7 } & 20 & 6.98 & 10 & 0 & 5 & 45 \\
\cline { 2 - 7 } & 40 & 6.42 & 10 & 0 & 12 & 38 \\
\cline { 2 - 7 } & 60 & 6.54 & 10 & 0 & 18 & 32 \\
\cline { 2 - 7 } & 80 & 7.14 & 10 & 0 & 19 & 31 \\
\hline \hline \multirow{5}{*}{$10^{-5}$} & 100 & 6.68 & 10 & 0 & 28 & 22 \\
\cline { 2 - 7 } & 10 & 13.16 & 18 & 0 & 3 & 47 \\
\cline { 2 - 7 } & 20 & 11.44 & 15 & 0 & 11 & 39 \\
\cline { 2 - 7 } & 40 & 11.88 & 15 & 0 & 11 & 39 \\
\cline { 2 - 7 } & 60 & 10.74 & 16 & 0 & 21 & 29 \\
\cline { 2 - 7 } & 10 & 10.54 & 16 & 0 & 23 & 27 \\
\hline
\end{tabular}


Tabela 1.3: Comportamento de MEQB para o caso de ponto de sela

\begin{tabular}{|c|c|c|c|c|c|c|}
\hline \multirow{3}{*}{$\sigma_{1}=\sigma_{2}$} & \multirow{2}{*}{ dimensão } & \multicolumn{2}{|c|}{ número de iterações } & \multicolumn{3}{|c|}{ número de vezes que é satisfeito } \\
\cline { 2 - 7 } & média & máximo & critério 1 & critério 2 & critério 3 \\
\hline \hline \multirow{4}{*}{$10^{-1}$} & 10 & 2.24 & 8 & 0 & 0 & 50 \\
\cline { 2 - 7 } & 20 & 2.08 & 4 & 0 & 0 & 50 \\
\cline { 2 - 7 } & 40 & 2.90 & 4 & 0 & 0 & 50 \\
\cline { 2 - 7 } & 60 & 3.10 & 4 & 0 & 0 & 50 \\
\cline { 2 - 7 } & 80 & 3.34 & 5 & 0 & 0 & 50 \\
\cline { 2 - 7 } & 100 & 3.50 & 5 & 0 & 0 & 50 \\
\hline \hline \multirow{5}{*}{$10^{-3}$} & 10 & 7.26 & 13 & 1 & 0 & 49 \\
\cline { 2 - 7 } & 20 & 6.84 & 10 & 0 & 0 & 50 \\
\cline { 2 - 7 } & 40 & 6.92 & 9 & 0 & 0 & 50 \\
\cline { 2 - 7 } & 60 & 6.70 & 10 & 0 & 0 & 50 \\
\cline { 2 - 7 } & 80 & 6.66 & 9 & 0 & 0 & 50 \\
\cline { 2 - 7 } & 100 & 6.66 & 10 & 0 & 0 & 50 \\
\hline \hline \multirow{5}{*}{-5} & 10 & 14.04 & 18 & 0 & 0 & 50 \\
\cline { 2 - 7 } & 20 & 13.28 & 16 & 0 & 0 & 50 \\
\cline { 2 - 7 } & 40 & 12.84 & 15 & 0 & 0 & 50 \\
\cline { 2 - 7 } & 60 & 12.74 & 16 & 0 & 0 & 50 \\
\cline { 2 - 7 } & 80 & 12.62 & 15 & 0 & 0 & 50 \\
\cline { 2 - 7 } & 100 & 11.94 & 16 & 0 & 0 & 50 \\
\hline
\end{tabular}

Tabela 1.4: Comportamento de MEQB para o caso definido positivo

\begin{tabular}{|c|c|c|c|c|c|c|}
\hline \multirow{3}{*}{$\sigma_{1}=\sigma_{2}$} & \multirow{2}{*}{ dimensão } & \multicolumn{2}{|c|}{ número de iterações } & \multicolumn{3}{|c|}{ número de vezes que é satisfeito } \\
\cline { 2 - 7 } & média & máximo & critério 1 & critério 2 & critério 3 \\
\hline \hline \multirow{4}{*}{$10^{-1}$} & 10 & 2.08 & 3 & 42 & 7 & 1 \\
\cline { 2 - 7 } & 20 & 2.26 & 4 & 28 & 22 & 0 \\
\cline { 2 - 7 } & 40 & 2.30 & 4 & 28 & 20 & 2 \\
\cline { 2 - 7 } & 60 & 2.52 & 4 & 19 & 28 & 3 \\
\cline { 2 - 7 } & 80 & 2.58 & 4 & 18 & 29 & 3 \\
\cline { 2 - 7 } & 100 & 2.66 & 4 & 11 & 36 & 3 \\
\hline \hline \multirow{5}{*}{$10^{-3}$} & 10 & 2.38 & 4 & 38 & 12 & 0 \\
\cline { 2 - 7 } & 20 & 2.58 & 5 & 34 & 16 & 0 \\
\cline { 2 - 7 } & 40 & 3.08 & 5 & 19 & 31 & 0 \\
\cline { 2 - 7 } & 60 & 3.10 & 6 & 24 & 26 & 0 \\
\cline { 2 - 7 } & 80 & 3.66 & 5 & 14 & 36 & 0 \\
\hline \hline \multirow{5}{*}{$10^{-5}$} & 100 & 3.52 & 5 & 13 & 37 & 0 \\
\cline { 2 - 7 } & 10 & 2.40 & 5 & 41 & 9 & 0 \\
\cline { 2 - 7 } & 20 & 2.82 & 5 & 31 & 19 & 0 \\
\cline { 2 - 7 } & 40 & 3.02 & 5 & 30 & 20 & 0 \\
\cline { 2 - 7 } & 60 & 3.48 & 6 & 22 & 28 & 0 \\
\cline { 2 - 7 } & 100 & 4.02 & 6 & 12 & 38 & 0 \\
\hline
\end{tabular}


Note que, mesmo no caso geral, o critério 3 de convergência é satisfeito em um grande número de vezes, principalmente quando os valores de $\sigma_{1}$ são maiores. Veja na tabela 1.1 que, para $\sigma_{1}=10^{-1}$, a maioria das instâncias termina com este critério. A medida que o valor de $\sigma_{1}$ decresce, aumenta o número de instâncias em que o critério 2 é satisfeito.

No caso difícil, como já se esperava, para a maioria das instância, o Algoritmo 2 pára no critério 3. Mas, note que, mais uma vez, quanto menor o valor de $\sigma_{1}$, maior o número de instâncias que param no critério 2. No entanto, a "migração" de instâncias de um critério para o outro não é tão grande quanto no caso geral. Olhando para a tabela 1.2 pode-se observar que a diferença entre o número de instâncias que satisfazem o critério 2 e o critério 3 , quando se muda o valor de $\sigma_{1}$ de $10^{-3}$ para $10^{-5}$, não varia muito.

O caso de ponto de sela é mais radical: apenas em 1 das instâncias o critério 1 foi satisfeito. Isso porque, apenas neste caso, a matriz $B$ gerada já era definida positiva e, como $g=0$, o minimizador da quadrática é 0 . Nas demais instâncias isso não aconteceu e, portanto, como o passo $p$ obtido sempre é igual a 0 , é necessário fazer uso do vetor $z$. E, neste caso, o critério a ser satisfeito deve ser o 3 .

No caso definido positivo é interessante notar que, ao contrário de todos os demais casos, independentemente do valor de $\sigma_{1}$, o número de iterações gastos até a convergência praticamente não varia. Além disso, este é o único caso em que a quantidade de instâncias que param pelo critério 1 é significativo. Quando o valor de $\sigma_{1}$ é maior, algumas (poucas) instâncias satisfazem o critério 3. Mas, independente do valor de $\sigma_{1}$, o número de instâncias que por fim satisfazem os critérios 1 e 2 se dividem: praticamente metade delas satisfaz um critério e a outra metade satisfaz o outro.

Em [18] é apresentado o comportamento da implementação do Algoritmo 2 feita pelos autores. O comportamento de MEQB é igual ao apresentado em [18]. Os relatos sobre a variação do número de iterações e do critério de convergência satisfeito para cada instância com relação à variação do valor de $\sigma_{1}$ é o mesmo observado em nossos experimentos. 


\section{Capítulo 2}

\section{Regiões de confiança}

Como já mencionado anteriormente, estamos interessados na construção de um método para minimização de funções irrestritas baseado em regiões de confiança. Para isso, o algoritmo apresentado no capítulo 1 será usado para a resolução dos subproblemas que aparecem em cada iteração do método de regiões de confiança.

A técnica de regiões de confiança para a minimização de uma função irrestrita $f$ é baseada na seguinte idéia: dado um ponto $x^{k}$, construímos uma aproximação quadrática de $f$. Estabelecemos uma região de confiança de raio $\Delta^{k}$ em torno de $x^{k}$ para a qual acreditamos que a aproximação quadrática está próxima da função $f$. Então, encontramos o minimizador $s^{k}$ desta aproximação nesta região. Depois de calculado $s^{k}$, verificamos se "andando" na direção $s^{k}$ (ou seja, em $x^{k}+s^{k}$ ) obtemos decréscimo suficiente na função $f$. Se isso acontecer, passamos ao ponto $x^{k}+s^{k}$, aumentando ou não o raio $\Delta^{k}$ da região de confiança, e inicia-se uma nova iteração. Se o decréscimo obtido em $f$ não for suficiente, diminui-se o raio $\Delta^{k}$ da região e calcula-se um novo $s^{k}$.

Neste capítulo apresentaremos detalhadamente a técnica de regiões de confiança. Mostraremos um algoritmo baseado nesta técnica para a minimização de funções irrestritas usando o método apresentado no capítulo 1 para a resolução do subproblema que aparece a cada iteração. Apresentamos os resultados numéricos da aplicação da nossa implementação deste algoritmo nas 18 funções de teste utilizadas em [18] e introduzidas em [17].

\subsection{O algoritmo baseado em regiões de confiança}

A aproximação quadrática de uma função $f$ em $C^{2}$, no ponto $x^{k}$, é dada pela série de Taylor truncada de $f\left(x^{k}+w\right)$ de ordem 2. Ou seja,

$$
f\left(x^{k}+w\right) \approx f\left(x^{k}\right)+\varphi_{k}(w)
$$

onde

$$
\varphi_{k}(w)=\frac{1}{2} w^{T} \nabla^{2} f\left(x^{k}\right) w+\nabla f\left(x^{k}\right)^{T} w
$$


A técnica de regiões de confiança para a minimização de uma função irrestrita $f$ é baseada na seguinte idéia: dado um ponto $x^{k}$, construímos uma aproximação quadrática $f\left(x^{k}\right)+$ $\varphi_{k}(w)$ de $f$. Estabelecemos uma região de confiança de raio $\Delta^{k}$ em torno de $x^{k}$ para a qual acreditamos que a aproximação quadrática está próxima da função $f$. Então, encontramos o minimizador $s^{k}$ desta aproximação nesta região, ou seja, queremos $s^{k}$ que resolve

$$
\begin{array}{ll}
\text { minimizar } & f\left(x^{k}\right)+\varphi_{k}(w) \\
\text { sujeita a } & \|w\| \leq \Delta^{k} .
\end{array}
$$

Calculado $s^{k}$, verificamos se "andando" na direção $s^{k}$ (ou seja, em $x^{k}+s^{k}$ ) obtemos decréscimo suficiente na função $f$. Se isso acontecer, passamos ao ponto $x^{k}+s^{k}$, aumentando ou não o raio $\Delta^{k}$ da região de confiança, e inicia-se uma nova iteração. Se o decréscimo obtido em $f$ não for suficiente, diminui-se o raio $\Delta^{k}$ da região e calcula-se um novo $s^{k}$.

O Algoritmo 3 mostra a forma geral de um método para minimização irrestrita baseado em regiões de confiança.

Algoritmo 3 Dadas as constantes $0<\mu<\eta<1$ e $0<\gamma_{1}<\gamma_{2}<1<\gamma_{3}$. Dados $x^{0} \in \mathbb{R}^{n}$ $e \Delta^{0}>0$.

Passo 1. $k=0$

Passo 2. Calcule $\nabla f\left(x^{k}\right)$ e $\nabla^{2} f\left(x^{k}\right)$

Passo 3. Se o "critério de convergência foi satisfeito" então

Pare e devolva $x^{k}$ como solução

Passo 4. Calcule $s^{k}$ uma solução aproximada de (2.1)

Passo 5. Calcule $\rho^{k}=\frac{f\left(x^{k}+s^{k}\right)-f\left(x^{k}\right)}{\varphi_{k}\left(s^{k}\right)}$

Passo 6. Se $\rho^{k} \leq \mu$ então

$$
\Delta^{k}=\Delta \in\left[\gamma_{1} \Delta^{k}, \gamma_{2} \Delta^{k}\right]
$$

Volte para o Passo 4

Passo 7. $x^{k+1}=x^{k}+s^{k}$

Passo 8. Se $\rho^{k} \leq \eta$ então

$$
\Delta^{k+1} \in\left[\gamma_{2} \Delta^{k}, \Delta^{k}\right]
$$

Senão

$$
\Delta^{k+1} \in\left[\Delta^{k}, \gamma_{3} \Delta^{k}\right]
$$

Passo 9. $k=k+1$ 


\section{Passo 10. Volte para o Passo 2}

É claro que estamos interessados em utilizar o Algoritmo 2 para resolver o Passo 4. Vejamos agora que isto é possível.

Primeiro vejamos quais condições a solução aproximada $s^{k}$ de (2.1) deve satisfazer para garantir que o Algoritmo 3 gere uma seqüência $\left\{x^{k}\right\}$ convergente a um ponto $x^{*} \operatorname{com} \nabla f\left(x^{*}\right)=0$ e $\nabla^{2} f\left(x^{*}\right)$ semidefinida positiva.

Para isso, exige-se que exista $\beta \in(0,1)$ tal que

$$
-\varphi\left(s^{k}\right) \geq \beta \max \left\{-\varphi(w): w=\alpha \nabla f\left(x^{k}\right),\|w\| \leq \Delta^{k}\right\}, \quad\left\|s^{k}\right\| \leq \Delta^{k} .
$$

Sob esta condição, provou-se em [23] que, se $\mu>0$, toda seqüência $\left\{\nabla f\left(x^{k}\right)\right\}$ converge a 0 . Com isso, podemos esperar que $\left\{x^{k}\right\}$ vá convergir a um ponto $x^{*} \operatorname{com} \nabla f\left(x^{*}\right)=0$.

Além disso, se temos $\sigma \in(0,1)$ tal que

$$
\varphi_{k}\left(s^{k}\right)=\min \left\{\varphi_{k}(w):\|w\| \leq \delta^{k}\right\},
$$

com

$$
\left\|s^{k}\right\| \leq \delta^{k} \in\left[(1-\sigma) \Delta^{k},(1+\sigma) \Delta^{k}\right]
$$

temos que $\nabla^{2} f\left(x^{*}\right)$ é semidefinida positiva (veja [22]).

Como o critério de convergência (1.8) pode não ser consistente com essas condições, podemos usar a seguinte generalização

$$
-\varphi_{k}\left(s^{k}\right) \geq \beta_{1}\left|\varphi_{k}^{*}\right| \operatorname{com}\left\|s^{k}\right\| \leq \beta_{2} \Delta^{k}
$$

$\operatorname{com} \beta_{1}>0$ e $\beta_{2}>0$.

Por (1.9) temos que

$$
\varphi_{k}\left(s^{k}\right)-\varphi_{k}^{*} \leq \sigma_{1}\left(2-\sigma_{1}\right) \max \left\{\left|\varphi_{k}^{*}\right|, \sigma_{2}\right\}, \quad\left\|s^{k}\right\| \leq\left(1+\sigma_{1}\right) \Delta^{k} .
$$

Se $\varphi_{k}^{*} \neq 0$ e $\sigma_{2}=0$, temos que

$$
\varphi_{k}\left(s^{k}\right)-\varphi_{k}^{*} \leq \sigma_{1}\left(2-\sigma_{1}\right)\left|\varphi_{k}^{*}\right|, \quad\left\|s^{k}\right\| \leq\left(1+\sigma_{1}\right) \Delta^{k} \Rightarrow
$$




$$
\begin{gathered}
-\varphi_{k}\left(s^{k}\right) \geq-\varphi_{k}^{*}-\sigma_{1}\left(2-\sigma_{1}\right)\left|\varphi_{k}^{*}\right|, \quad\left\|s^{k}\right\| \leq\left(1+\sigma_{1}\right) \Delta^{k} \Rightarrow \\
-\varphi_{k}\left(s^{k}\right) \geq\left|\varphi_{k}^{*}\right|-\sigma_{1}\left(2-\sigma_{1}\right)\left|\varphi_{k}^{*}\right|, \quad\left\|s^{k}\right\| \leq\left(1+\sigma_{1}\right) \Delta^{k} \Rightarrow \\
-\varphi_{k}\left(s^{k}\right) \geq\left(1-\sigma_{1}\right)^{2}\left|\varphi_{k}^{*}\right|, \quad\left\|s^{k}\right\| \leq\left(1+\sigma_{1}\right) \Delta^{k} .
\end{gathered}
$$

Tomando-se $\beta_{1}=\left(1-\sigma_{1}\right)^{2}$ e $\beta_{2}=\left(1+\sigma_{1}\right)$, temos que o Algoritmo 2 satisfaz as condições requeridas.

Caso $\varphi^{*}=0$, temos que $\nabla f\left(x^{k}\right)=0$ e $\nabla^{2} f\left(x^{k}\right)$ é semidefinida positiva, e o Algoritmo 2 pára com $x^{k}$ como solução.

Portanto, podemos utilizar o Algoritmo 2, com $\sigma_{2}=0$, para resolver o Passo 4 do Algoritmo 3 .

O Algoritmo 4 mostra a versão final do método para minimização irrestrita baseado em regiões de confiança, com todos os detalhes não especificados pelo Algoritmo 3.

Algoritmo 4 Dados $\eta, \epsilon, \sigma \in(0,1), x^{0} \in \mathbb{R}^{n}$ e $\Delta^{0}>\Delta_{\min }>0$

Passo 1. $k=0$

Passo 2. $\lambda^{0}=0$

Passo 3. Calcule $\nabla f\left(x^{k}\right)$ e $\nabla^{2} f\left(x^{k}\right)$

Passo 4. Se $\left\|\nabla f\left(x^{k}\right)\right\|_{\infty} \leq \epsilon$ então

Pare e devolva $x^{k}$ como solução

Passo 5. Calcule $s^{k}$ usando o Algoritmo 2, com os parâmetros:

$$
\begin{aligned}
& \nabla^{2} f\left(x^{k}\right) \text { (como a matriz simétrica), } \\
& \nabla f\left(x^{k}\right)\left(\text { como o vetor em } \mathbb{R}^{n}\right), \\
& \Delta^{k}, \lambda^{k}, \sigma_{1}=\sigma \text { e } \sigma_{2}=0
\end{aligned}
$$

Passo 6. Calcule $\rho^{k}=\frac{f\left(x^{k}+s^{k}\right)-f\left(x^{k}\right)}{\varphi^{k}\left(s^{k}\right)}$

Passo 7. Se $\rho^{k} \leq 0.25$ então

$$
\Delta^{k}=\max \left\{\frac{\left\|s^{k}\right\|}{4}, \Delta_{\min }\right\}
$$

Passo 8. Se $\rho^{k} \geq 0.5$ e $\left\|s^{k}\right\| \approx \Delta^{k}$ então 


$$
\Delta^{k}=2 \Delta^{k}
$$

Passo 9. Se $\rho^{k}<\eta$ então

\section{Volte para o Passo 5}

Passo 10. $\Delta^{k+1}=\max \left\{\Delta^{k}, \Delta_{\min }\right\}$

Passo 11. $x^{k+1}=x^{k}+s^{k}$

Passo 12. Atualize $\lambda^{k+1}$ com o valor de $\lambda$ obtido como solução no Passo 5

Passo 13. $k=k+1$

Passo 14. Volte para o Passo 3

Um detalhe importante é que, quando resolvemos um problema do tipo (2.1), podemos utilizar o valor de $\lambda^{k}$, resultante da aplicação do Algoritmo 2, como valor inicial de $\lambda$ para a próxima chamada a este algoritmo. Isso porque o valor $\lambda^{k}$ é um limitante inferior para a resolução do problema com os mesmos parâmetros, mas com um valor de $\Delta^{k}$ menor.

Em [18] temos a prova de que, usando $\eta \in(0,0.25)$ no Algoritmo 4, temos $\lim _{k \rightarrow \infty}\left\|\nabla f\left(x^{k}\right)\right\|$ $=0$. Mais ainda, se $C=\left\{x: f(x) \leq f\left(x^{0}\right)\right\}$ for compacto então há duas alternativas: ou o Algoritmo 4 termina num ponto $x^{k} \in C$ que satisfaz as condições necessárias de segunda ordem para que $x^{k}$ seja o minimizador de $f$ (ou seja, $\nabla f\left(x^{k}\right)=0$ e $\nabla^{2} f\left(x^{k}\right)$ é semidefinida positiva); ou a seqüência $\left\{x^{k}\right\}$ gerada pelo Algoritmo 4 tem um ponto limite $x^{*} \in C$ para o qual valem as condições necessárias de segunda ordem.

Em [18] também se afirma que o grau de convergência do Algoritmo 4 é Q-superlinear.

\subsection{Resultados numéricos}

Implementamos o Algoritmo 4 numa subrotina em FORTRAN 77 chamada MEQBRC. Para verificar o comportamento desta subrotina, foram utilizadas as 18 funções sugeridas em [17] para teste de métodos de minimização irrestrita. São elas:

1. Helical valley function

2. Biggs EXP6 function

3. Gaussian function

4. Powell badly scaled function

5. Box three-dimensional function

6. Variably dimensioned function

7. Watson function

8. Penalty function I

9. Penalty function II

10. Brown badly scaled function

11. Brown and Dennis function 
12. Gulf research and development function

13. Trigonometric function

14. Extended Rosenbrock function

15. Extended Powell singular function

16. Beale function

17. Wood function

18. Chebyquad function

$\mathrm{Na}$ tabela 2.1 pode ser verificado o comportamento da subrotina quando aplicada a cada uma das funções acima citadas, com os parâmetros $\eta=10^{-1}, \epsilon=10^{-4}, \sigma=0.1 \mathrm{e}$ $\Delta_{\min }=10^{-3}$.

A cada iteração são feitas exatamente uma avaliação de gradiente e uma avaliação de hessiana. Portanto, tanto o número de avaliações de gradiente quanto o número de avaliações de hessiana é igual ao número de iterações.

Tabela 2.1: Comportamento de MEQBRC para funções gerais

\begin{tabular}{|c|c|c|c|c|}
\hline \multirow{2}{*}{ função } & número de & número de avaliações & \multicolumn{2}{|c|}{ mínimo } \\
\cline { 4 - 5 } iterações & de função & obtido & esperado \\
\hline \hline 1 & 9 & 20 & $4.2668906 \times 10^{-17}$ & 0 \\
\hline 2 & 4331 & 9626 & $1.9781722 \times 10^{-04}$ & 0 \\
\hline 3 & 1 & 2 & $1.1292712 \times 10^{-08}$ & $1.12793 \ldots \times 10^{-08}$ \\
\hline 4 & 414 & 834 & $2.8396414 \times 10^{-08}$ & 0 \\
\hline 5 & 18 & 36 & $4.2190293 \times 10^{-09}$ & 0 \\
\hline 6 & 106 & 364 & $2.3891072 \times 10^{-08}$ & 0 \\
\hline 7 & 1343 & 3650 & $7.7074693 \times 10^{-03}$ & $2.28767 \ldots \times 10^{-03}$ \\
\hline 8 & 10 & 20 & $2.4089543 \times 10^{-05}$ & $2.24997 \ldots \times 10^{-05}$ \\
\hline 9 & 9 & 28 & $9.5186103 \times 10^{-06}$ & $9.37629 \ldots \times 10^{-06}$ \\
\hline 10 & 35 & 74 & $1.3509536 \times 10^{-27}$ & 0 \\
\hline 11 & 36 & 80 & $8.5822202 \times 10^{+04}$ & $8.58222 \ldots \times 10^{+04}$ \\
\hline 12 & 34 & 70 & $5.5319044 \times 10^{-13}$ & 0 \\
\hline 13 & 19 & 56 & $2.3674882 \times 10^{-09}$ & 0 \\
\hline 14 & 23 & 50 & $1.9317734 \times 10^{-16}$ & 0 \\
\hline 15 & 1931 & 10226 & $5.8417645 \times 10^{-06}$ & 0 \\
\hline 16 & 6 & 14 & $2.9592252 \times 10^{-13}$ & 0 \\
\hline 17 & 44 & 96 & $7.4953421 \times 10^{-19}$ & 0 \\
\hline 18 & 19 & 54 & $5.0992633 \times 10^{-10}$ & 0 \\
\hline
\end{tabular}

Note que MEQBRC obteve excelentes resultados. O mínimo encontrado é muitíssimo próximo do esperado. Além de o número de iterações (e, conseqüentemente, de avaliações de gradiente e hessianas) e de avaliações de funções ser, na maioria dos casos, muito pequeno. 


\section{Capítulo 3}

\section{O método do Gradiente Espectral Projetado}

No método de restrições ativas proposto em [2], cada ponto candidato a solução obtido pelo algoritmo pertence a uma face da caixa (restrições do problema) e, a cada iteração, precisamos fazer uma minimização da função objetivo numa dessas faces. Para isso, não utilizaremos o método de regiões de confiança puro, apresentado no capítulo 2. Utilizaremos, sim, um algoritmo que mistura a técnica de regiões de confiança e o método do Gradiente Espectral Projetado (veja [3], [4] e [5]).

Além disso, quando o método de restrições ativas decide mudar de face, ou seja, decide mudar o conjunto de restrições ativas corrente, usamos o método do Gradiente Espectral Projetado (chamado de SPG) para definir qual será a nova face a ser trabalhada. Em outras palavras, usamos o método do Gradiente Espectral Projetado para escolher quais restrições devem entrar no conjunto das restrições ativas e quais devem sair deste conjunto. O funcionamento do método de restrições ativas e o papel do SPG neste método ficarão mais claros nos capítulos 4 e 5 .

O SPG foi escolhido por ter fácil implementação e ser eficiente. Isso porque apenas informação de primeira ordem é necessária para a execução deste método. Outro fator importante é que, pelo fato de se basear em projeções, o SPG tende a acrescentar um grande número de restrições ao conjunto de restrições ativas. Isso é muito interessante, pois, quanto maior o número de restrições ativas, menor a dimensão do subproblema a ser resolvido na face.

Na seção 3.1 apresentamos o método do Gradiente Espectral Projetado, utilizado para minimizar funções gerais restritas a uma caixa, proposto em [4]. As provas de convergência do método podem ser encontradas em [4]. Na seção 3.2 apresentamos os detalhes de nossa implementação deste método.

\subsection{O algoritmo}

O método do Gradiente Espectral Projetado é usado para resolver o seguinte problema: 


$$
\begin{array}{ll}
\text { minimizar } & f(x) \\
\text { sujeita a } & x \in A,
\end{array}
$$

onde $f$ é uma função em $C^{1}, x \in \mathbb{R}^{n}$ e $A$ é um conjunto convexo fechado.

Estamos interessados no caso em que $A$ é uma caixa, ou seja, $l \leq x \leq u, \operatorname{com} l, u, x \in \mathbb{R}^{n}$.

A idéia do algoritmo é a seguinte: na iteração $k$, calculamos uma direção de descida $d^{k}$ e fazemos uma busca linear para que $x^{k}+t_{k} d^{k}$ satisfaça a condição de Armijo. Quando isso acontece, temos o novo ponto $x^{k+1}=x^{k}+t_{k} d^{k} \in A$. A direção $d^{k}$ é calculada com base na projeção ortogonal de $-\lambda_{k}^{\mathrm{SPG}} \nabla f\left(x^{k}\right)$ no conjunto $A$, onde $\lambda_{k}^{\mathrm{SPG}}$ (chamado de passo espectral) é um escalar calculado a partir de informações fornecidas pelo ponto atual $x^{k}$ e pelo ponto $x^{k-1}$ da iteração anterior.

Antes de apresentar uma típica iteração do algoritmo, vejamos algumas notações: dado um ponto $x \in \mathbb{R}^{n}$, denotamos por $P_{A}(x)$ a projeção ortogonal do ponto $x$ no conjunto $A$; dado um conjunto $A$, denotamos por $A^{0}$ o conjunto de pontos interiores de $A$.

Iteração do SPG Dados $x^{k} \in A^{0}, 0<\lambda_{\min }^{S P G}<\lambda_{\max }^{S P G}<\infty, \alpha \in\left(0, \frac{1}{2}\right)$. Supondo que $\nabla f\left(x^{k}\right) \neq 0$.

Passo 1. Calcule $s_{k}=x^{k}-x^{k-1}$

Passo 2. Calcule $y_{k}=\nabla f\left(x^{k}\right)-\nabla f\left(x^{k-1}\right)$

Passo 3. Cálculo do $\lambda_{k}^{S P G}$

Se $s_{k}^{T} y_{k} \leq 0$ então

$$
\lambda_{k}^{\mathrm{SPG}}=\lambda_{\max }^{\mathrm{SPG}}
$$

\section{Senão}

$$
\lambda_{k}^{\mathrm{SPG}}=\min \left\{\lambda_{\max }^{\mathrm{SPG}}, \max \left\{\lambda_{\min }^{\mathrm{SPG}}, \frac{s_{k}{ }^{T} s_{k}}{s_{k} y_{k}}\right\}\right\}
$$

Passo 4. Calcule $d^{k}=P_{A}\left(x^{k}-\lambda_{k}^{\mathrm{SPG}} \nabla f\left(x^{k}\right)\right)-x^{k}$

Passo 5. $t_{k}=1$

Passo 6. Se $f\left(x^{k}+t_{k} d^{k}\right) \leq f\left(x^{k}\right)+\alpha t_{k} \nabla f\left(x^{k}\right)^{T} d^{k}$ então

Termine a iteração com $y^{k}=x^{k}+t_{k} d^{k}$

\section{Senão}

Escolha $t_{\text {novo }} \in\left[0.1 t_{k}, 0.5 t_{k}\right]$

Faça $t_{k}=t_{\text {novo }}$

Repita o Passo 6 
O valor obtido para $\lambda_{k}^{\text {SPG }}$ possui alguma informação de segunda ordem da função objetivo (veja [4]). Na verdade, temos que

$$
\left(\frac{1}{\lambda_{k}^{S P G}}\right) I=\begin{array}{ll}
\operatorname{argmin}_{D} & \left\|D s_{k}-y_{k}\right\| \\
\text { sujeita a } & D=\gamma I,
\end{array}
$$

onde $I$ é a matriz identidade. Ou seja, $\left(\frac{1}{\lambda_{k}^{S P G}}\right) I$ é a matriz diagonal que melhor aproxima a equação secante (equação esta que é uma aproximação da hessiana da função objetivo).

Quanto à convergência do algoritmo do Gradiente Espectral Projetado, temos que todo ponto de acumulação da seqüência $\left\{x^{k}\right\}$ gerada por ele é um ponto estacionário restrito. Para a prova, veja [4].

\subsection{Implementação}

Implementamos o algoritmo SPG em FORTRAN 77. Para implementar este método, foi necessário escolher uma maneira para se calcular o passo espectral inicial $\lambda_{0}^{S P G}$, já que este não pode ser calculado da mesma maneira que os demais passos espectrais. Além disso, para que todos os passos estejam perfeitamente definidos, precisamos definir uma maneira para escolher $t_{\text {novo }}$ no Passo 3 da Iteração do SPG. Nas seções abaixo, apresentamos a maneira utilizada em nossa implementação para se calcular $\lambda_{0}^{\mathrm{SPG}}$ e $t_{\text {novo }}$.

\subsubsection{Cálculo de $\lambda_{0}^{\text {SPG }}$}

Uma maneira de calcular $\lambda_{0}^{\text {SPG }}$ foi proposta em [3]. A idéia é calcular um $\bar{x}$ dando um passo muito pequeno a partir de $x^{0}$ na direção de $-\nabla f\left(x^{0}\right)$. Neste caso, esperamos que a função objetivo decresça e satisfaça a condição de Armijo. Por isso utilizamos, para calcular $\lambda_{0}^{\mathrm{SPG}}$, os mesmos passos usados para calcular $\lambda_{k}^{\mathrm{SPG}}$, considerando $\bar{x}$ como $x^{k}$ e $x^{0}$ como $x^{k-1}$. Abaixo estão descritos todos os passos necessários para o cálculo de $\lambda_{0}^{\mathrm{SPG}}$ (dados $\epsilon_{\text {rel }}, \epsilon_{a b s}>0$ ):

Passo 1. Calcule $t_{p e q}=\max \left\{\epsilon_{r e l}\left\|x^{0}\right\|_{\infty}, \epsilon_{a b s}\right\}$

Passo 2. Calcule $\bar{x}=x^{0}-t_{p e q} \nabla f\left(x^{0}\right)$

Passo 3. Calcule $\bar{s}=\bar{x}-x^{0}$

Passo 4. Calcule $\bar{y}=\nabla f(\bar{x})-\nabla f\left(x^{0}\right)$

Passo 5. Cálculo do $\lambda_{0}^{S P G}$

Se $\bar{s}^{T} \bar{y} \leq 0$ então $\lambda_{0}^{\mathrm{SPG}}=\lambda_{\max }^{\mathrm{SPG}}$ 
Senão

$$
\lambda_{0}^{\mathrm{SPG}}=\min \left\{\lambda_{\max }^{\mathrm{SPG}}, \max \left\{\lambda_{\min }^{\mathrm{SPG}}, \frac{\bar{s}^{T} \bar{s}}{\bar{s}^{T} \tilde{y}}\right\}\right\}
$$

\subsubsection{Escolha de $t_{\text {novo }}$}

Uma forma simples é calcular $t_{\text {novo }}=\frac{t_{k}}{2}$. Uma forma mais interessante é utilizar a interpolação quadrática uni-dimensional $q(w)$ tal que $q(0)=f\left(x^{k}\right), q\left(t_{k}\right)=f\left(x^{k}+t_{k} d^{k}\right)$ e $\nabla q(0)=\nabla f\left(x^{k}\right)^{T} d^{k}$. Para obter $t_{n o v o}$, calculamos o mínimo de $q(w)$ e fazemos a salvaguarda (lembre-se que estamos interressados em $t_{\text {novo }} \in\left[0.1 t_{k}, 0.5 t_{k}\right]$ ).

Primeiro, vejamos como calcular o mínimo de $q(w)$. Observe que

$$
\begin{gathered}
q(w)=\frac{1}{2} a w^{2}+b w+c \\
q^{\prime}(w)=a w+b .
\end{gathered}
$$

Por (3.1), temos que $q(0)=c$. Como $q(0)=f\left(x^{k}\right)$, temos

$$
c=f\left(x^{k}\right) .
$$

Por $(3.2)$, temos $q^{\prime}(0)=b$. Como $q^{\prime}(0)=\nabla f\left(x^{k}\right)^{T} d^{k}$, temos

$$
b=\nabla f\left(x^{k}\right)^{T} d^{k} .
$$

Ainda por (3.1), temos $q\left(t_{k}\right)=\frac{1}{2} a t_{k}^{2}+b t_{k}+c$. Substituindo $b$ e $c$ pelos valores obtidos, temos

$$
\begin{gathered}
q\left(t_{k}\right)=\frac{1}{2} a t_{k}^{2}+t \nabla f\left(x^{k}\right)^{T} d^{k}+f\left(x^{k}\right)=f\left(x^{k}+t_{k} d^{k}\right) \Rightarrow \\
a=2 \frac{f\left(x^{k}+t_{k} d^{k}\right)-f\left(x^{k}\right)-t_{k} \nabla f\left(x^{k}\right)^{T} d^{k}}{t_{k}^{2}}
\end{gathered}
$$

Como estamos interessados no mínimo de $q(w)$, queremos $q^{\prime}\left(t_{\text {novo }}\right)=0$. Ou seja,

$$
\begin{gathered}
q^{\prime}\left(t_{\text {novo }}\right)=a t_{\text {novo }}+b=0 \Rightarrow t_{\text {novo }}=-\frac{b}{a} \Rightarrow \\
t_{\text {novo }}=-\frac{\nabla f\left(x^{k}\right)^{T} d^{k}}{2 \frac{f\left(x^{k}+t_{k} d^{k}\right)-f\left(x^{k}\right)-t_{k} \nabla f\left(x^{k}\right)^{T} d^{k}}{t_{k}^{2}}} \Rightarrow
\end{gathered}
$$




$$
t_{\text {novo }}=-\frac{t_{k}^{2} \nabla f\left(x^{k}\right)^{T} d^{k}}{2\left(f\left(x^{k}+t_{k} d^{k}\right)-f\left(x^{k}\right)-t_{k} \nabla f\left(x^{k}\right)^{T} d^{k}\right)} .
$$

A seguir estão os passos utilizados para a escolha de $t_{\text {novo }}$ utilizando a interpolação quadrática e salvaguarda:

Passo 1. Para $t_{k}$ pequeno não vale a pena calcular a interpolação quadrática

Se $t_{k} \leq 0.1$ então

$$
t_{\text {novo }}=\frac{t_{k}}{2}
$$

Passo 2. Calcule $t_{\text {tent }}=-\frac{t_{k}^{2} \nabla f\left(x^{k}\right)^{T} d^{k}}{2\left(f\left(x^{k}+t_{k} d^{k}\right)-f\left(x^{k}\right)-t_{k} \nabla f\left(x^{k}\right)^{T} d^{k}\right)}$

Passo 3. Salvaguarda de $t_{\text {novo }}$

$$
\begin{aligned}
\text { Se }\left(t_{\text {tent }} \geq 0.1\right) \text { e }\left(t_{\text {tent }} \leq 0.5 t_{k}\right) \text { então } \\
t_{\text {novo }}=t_{\text {tent }}
\end{aligned}
$$

Senão

$$
t_{\text {novo }}=\frac{t_{k}}{2}
$$

Um ponto interessante a se observar na salvaguarda do $t_{\text {novo }}$ é que não verificamos, como classicamente se faz, se $t_{\text {novo }} \in\left[0.1 t_{k}, 0.5 t_{k}\right]$, mas sim que $t_{\text {novo }} \in\left[0.1,0.5 t_{k}\right]$ (veja [5]). Isso pode ser interpretado da seguinte maneira: quando a interpolação rejeita $90 \%$ do intervalo original de busca pela solução $([0,1])$, julgamos que esta interpolação não é confiável e preferimos a opção $t_{n o v o}=\frac{t_{k}}{2}$. Este tipo de salvaguarda se mostrou mais eficiente do que a maneira clássica. 


\section{Capítulo 4}

\section{Um método híbrido para minimização nas faces}

O método de restrições ativas usa o esquema descrito em [2]. A caixa será dividida em "faces abertas". Em cada cada face aberta, um algoritmo interno é usado para modificar somente as variáveis livres. $\mathrm{O}$ algoritmo interno pode ser descrito como um método de ponto interior (irrestrito) que pode atingir a borda do conjunto convexo no qual a função objetivo está definida.

O algoritmo interno utilizado neste trabalho é baseado no método de regiões de confiança apresentado no capítulo 2. A dificuldade de implementar a estratégia de regiões de confiança usando norma euclidiana na presença de restrições de caixa está no fato de a intersecção de caixas e bolas não ser um conjunto simples. Por isso, encontrar o minimizador da aproximação quadrática em tal conjunto não é direta e a técnica de intersecção de algoritmos de regiões de confiança com restrições (veja [13] e [14]) não é fácil de aplicar.

Geralmente se diz que, quando temos problemas com restrições de caixa, regiões de confiança baseadas em norma infinito são melhores, porque elas apresentam a mesma geometria do domínio do problema (intersecção de caixas e caixas também são caixas). Entretanto, encontrar minimizadores globais de uma quadrática (não necessariamente convexa) não é tão simples quanto encontrar minimizadores de quadráticas arbitrárias em bolas (veja [11], [16] e [18]).

Portanto, regiões de confiança euclidianas comuns, cujos raios, em princípio, independem das restrições de caixa, merecem consideração. No entanto, as dificuldades técnicas devem ser eliminadas.

Neste capítulo propomos um algoritmo híbrido para minimização nas faces. Neste algoritmo usamos, basicamente, a técnica de regiões de confiança apresentada no capítulo 2 , modificada para lidar com o caso em que o minimizador da quadrática está fora da face. Quando estamos num ponto muito próximo da borda da face, usamos o método SPG, pois, neste caso, pode não valer a pena resolver o subproblema de regiões de confiança.

Apresentamos ainda o teorema (e prova) de convergência do algoritmo descrito na seção 
4.1 e mostramos os detalhes de nossa implementação.

\subsection{O algoritmo}

Seja $A \subset \mathbb{R}^{m}$ um conjunto fechado, limitado e convexo com interior não-vazio. Suponha que

$$
\bar{f}: \mathbb{R}^{m} \rightarrow \mathbb{R}
$$

tenha derivadas terceiras contínuas num conjunto aberto que contém $A$. Usaremos a seguinte notação:

$$
\bar{B}(x, \delta)=\left\{z \in \mathbb{R}^{m} \mid\|z-x\| \leq \delta\right\} .
$$

Lembrando que o subproblema de regiões de confiança, neste caso, pode ser escrito como

$$
\begin{array}{ll}
\operatorname{minimizar} & \bar{f}\left(x^{k}\right)+\varphi_{k}(x) \\
\text { sujeita a } & \|x\| \leq \Delta
\end{array}
$$

com

$$
\varphi_{k}(x)=\frac{1}{2} x^{T} \nabla^{2} \bar{f}\left(x^{k}\right) x+\nabla \bar{f}\left(x^{k}\right)^{T} x .
$$

Uma típica iteração do Algoritmo Interno é apresentada a seguir:

Iteração do Algoritmo Interno Dados $x^{k} \in A^{0} ; \Delta_{\min }>0 ; \Delta \geq \Delta_{\min }$

Passo 1. Cálculo da distância até a borda

Calcule $\Delta_{\text {borda }}=\max \left\{\bar{\Delta} \geq 0 \mid \bar{B}\left(x^{k}, \bar{\Delta}\right) \subset A\right\}$

Passo 2. Se está perto da borda, usa SPG

Se $\Delta_{\text {borda }}<2 \Delta_{\min }$ então

Se $\nabla \bar{f}\left(x^{k}\right)=0$ então

Termine a execução do Algoritmo Interno com $x^{k}$ declarando:

"Ponto estacionário de primeira ordem perto da borda"

Senão

Faça uma iteração do SPG para calcular $d^{k}$ e $y^{k}$ de forma que $y^{k}=x^{k}+t_{k} d^{k}$ (usando $\bar{f}$ e $\nabla \bar{f}$ no lugar de $f$ e $\nabla f$ )

Vá para o Passo 6 
Passo 3. Se está longe da borda, resolve o subproblema de regiões de confiança

Passo 3.1.

Calcule $\nabla^{2} \bar{f}\left(x^{k}\right)$

\section{Passo 3.2.}

Calcule $x^{\text {tent }}=x^{k}+d^{k}$ com $d^{k}$ solução do subproblema de regiões de confiança Se $\varphi_{k}\left(d^{k}\right)=0$ então

Termine a execução do Algoritmo Interno $\operatorname{com} x^{k}$ declarando:

"Ponto estacionário de segunda ordem"

Se $x^{\text {tent }} \notin A^{0}$ então

Calcule $t_{\text {max }}=\max \left\{t \in[0,1] \mid\left[x^{k}, x^{k}+t d^{k}\right] \subset A\right\}$

Se $\bar{f}\left(x^{k}+t_{\text {max }} d^{k}\right)<\bar{f}\left(x^{k}\right)$ então

Defina $y^{k}=x^{k}+t_{\text {max }} d^{k}$

Vá para o Passo 6

Senão

Escolha $\Delta \in\left[\Delta_{\min }, \Delta_{\text {borda }}\right)$

Volte para o Passo 3.2

Passo 4. Aceitação ou rejeição da solução do subproblema de regiões de confiança

Calcule $\rho^{k}=\frac{\bar{f}\left(x^{\text {tent }}\right)-\bar{f}\left(x^{k}\right)}{\varphi_{k}\left(x^{t e n t}\right)}$

Se $\rho^{k} \geq 0.1$ então

Defina $y^{k}=x^{\text {tent }}$

Senão

Escolha $\Delta \in\left[0.1\left\|d^{k}\right\|, 0.9\left\|d^{k}\right\|\right]$

Volte para o Passo 3.2

Passo 5. Atualização do raio da região de confiança

Escolha $\Delta \geq \Delta_{\min }$

Passo 6. Possivel melhora adicional

Escolha $x^{k+1} \in A$ tal que $\bar{f}\left(x^{k+1}\right) \leq \bar{f}\left(y^{k}\right)$

(Observe que $x^{k+1}=y^{k}$ é uma possível escolha)

Se $x^{k+1}$ pertence à borda de $A$ então

Termine a execução do Algoritmo Interno com $x^{k+1}$ declarando: 
"Iterando na borda"

No Passo 6, a possível escolha de $x^{k+1} \neq y^{k}$ tem, essencialmente, propósitos teóricos. Veremos a importância desta hipótese na prova do teorema de convergência na próxima seção. Entretanto, a liberdade permitida para a escolha de $x^{k+1}$ também pode ser usada para melhorar a iteração utilizando técnicas de extrapolação. Na seção 4.3 comentaremos o uso desta técnica.

Note que não é possível utilizar o método puro de regiões de confiança tal como apresentado do capítulo 2 para fazer a minimização nas faces. Perto da borda da face, algo diferente deve ser feito para se obter convergência. A estratégia utilizada pelo Algoritmo Interno é uma das possíveis estratégias que leva a propriedades de convergência adequadas. Para entender porque a estratégia de regiões de confiança sozinha não funciona, considere o problema

$$
\begin{array}{ll}
\operatorname{minimizar} & x_{1}+x_{2} \\
\text { sujeita a } & -1 \leq x_{1} \leq 100,0 \leq x_{2} \leq 100
\end{array}
$$

A solução deste problema é $(-1,0)$. Suponha que $x^{0}=(1,1)$ e que $x^{\text {tent }}=x^{k}+d^{k}$ com $d^{k}$ solução do subproblema de regiões de confiança definida pela distância de $x^{k}$ até a borda da face. O teste $\rho^{k} \geq 0.1$ é satisfeito por $x^{\text {tent }}$ para todo $k$, então podemos definir $x^{k+1}=x^{\text {tent }}$. No entanto, todos os iterandos estão na reta $x_{1}=x_{2}$, a seqüência é infinita e converge para $(0,0)$, que não é um ponto interior e $\nabla \bar{f}(0,0)=(1,1) \neq(0,0)$.

\subsection{Teorema de convergência}

Teorema 4.2.1 Na execução do Algoritmo Interno, ocorre uma e somente uma das seguintes possibilidades:

1. o algoritmo termina em um número finito de iterações num ponto $x^{k}$ tal que a distância de $x^{k}$ à borda é menor que $2 \Delta_{\min }$ e $\nabla \bar{f}\left(x^{k}\right)=0$;

2. o Algoritmo Interno termina em um número finito de iterações num ponto estacionário de segunda ordem $x^{k}$ tal que sua distância $\Delta_{\text {borda }} \geq 2 \Delta_{\text {min }}$;

3. o Algoritmo Interno termina depois de um número finito de iteraçôes num ponto $x^{k+1}$ na borda de $A$ e

$$
\bar{f}\left(x^{k+1}\right)<\bar{f}\left(x^{k}\right)<\ldots<\bar{f}\left(x^{0}\right)
$$

4. o Algoritmo Interno gera um seqüência infinita de iterandos. Neste caso: (a) todo ponto limite $x^{*}$ da seqüência gerada satisfaz $g_{I}\left(x^{*}\right)=0$ (veja a seção 5.1 para a definição de $\left.g_{I}\left(x^{k}\right)\right)$; (b) se um ponto limite $x^{*}$ é tal que sua distância até a borda é maior que $2 \Delta_{\text {min }}$ então $\nabla^{2} \bar{f}\left(x^{*}\right)$ é semidefinida positiva.

Prova As três primeiras possibilidades são consideradas na definição do algoritmo, já que elas correspondem aos três critérios de parada. Suponha, então, que uma seqüência infinita 
seja gerada e que $x^{*}$ é um ponto limite desta seqüência. Então, existe um conjunto infinito $K_{1} \subset \mathbb{N}$ tal que

$$
\lim _{k \in K_{1}} x^{k}=x^{*}
$$

Considere as duas possibilidades:

(a) Para infinitas iterações $k \in K_{2} \subset K_{1}, x^{k+1}$ é computado pelo Passo 2.

(b) Para infinitas iterações $k \in K_{3} \subset K_{1}, x^{k+1}$ é computado pelo Passo 3 .

No caso (a), os argumentos usados na prova de convergência do método do Gradiente Espectral Projetado (veja [4]) mostram que $g_{I}\left(x^{*}\right)=0$. Na verdade, se supomos que $\nabla \bar{f}\left(x^{k}\right) \neq 0$ e que $t_{k}$ fica longe de 0 , chegamos à contradição $\bar{f}\left(x^{k}\right) \rightarrow-\infty$. Por outro lado, se o ínfimo de $\left\{t_{k}\right\}$ é 0 , temos uma seqüência auxiliar $t_{k}^{\prime}$ que tende a 0 e tal que o teste feito no Passo 3 do SPG é rejeitado e então $t_{k}=t_{k}^{\prime}$. Isso implica, usando argumentos clássicos, que $g_{I}\left(x^{*}\right)=0$.

No caso (b), depois de renomeação, a seqüência pode ser interpretada como uma seqüência de iterandos de um método de regiões de confiança (com o raio de confiança inicial $\Delta$ maior ou igual a $\Delta_{\min }>0$ ), onde cada iterando é modificado com uma maior redução da função objetivo. Então, argumentos clássicos da literatura sobre regiões de confiança (veja, por exemplo, [13] e [14]) mostram que $\nabla \bar{f}\left(x^{*}\right)=0$ (o que implica que $g_{I}\left(x^{*}\right)=0$ ) e $\nabla^{2} \bar{f}\left(x^{*}\right)$ é semidefinida positiva.

Os argumentos acima mostram que qualquer ponto limite $x^{*}$ satisfaz $g_{I}\left(x^{*}\right)=0$. Mais ainda, se a distância de $x^{*}$ até a borda é maior que $2 \Delta_{\text {min }}$ então, para $k \in K_{1}$ suficientemente grande, $x^{k+1}$ é computado pelo Passo 3 . Portanto, o caso (b) se aplica e, então, a hessiana é semidefinida positiva.

\subsection{Implementação}

Implementamos o Algoritmo Interno em FORTRAN 77. Para que a implementação fosse possível, foi necessário definir como calcular alguns passos do Algoritmo Interno que foram deixados em aberto, a saber, como resolver o subproblema de regiões de confiança e como fazer as atualizações do raio da região de confiança. Além disso, nossa implementação utiliza a técnica de extrapolação apresentada em [2].

Para resolver subproblema de regiões de confiança do Passo 3.2, utilizamos nossa implementação do algoritmo apresentado em [18], o MEQB (veja capítulo 1), com os seguintes parâmetros: $\nabla^{2} \bar{f}\left(x^{k}\right)$ como a matriz simétrica $B, \nabla \bar{f}\left(x^{k}\right)$ como o vetor $g, \sigma_{1}=\sigma, \sigma_{2}=0$ e $\lambda^{0}=0$.

Nas seções a seguir apresentamos como foram feitas todas as atualizações do raio $\Delta$ da região de confiança e mostramos a técnica de extrapolação usada em nossa implementação. 


\subsubsection{Atualizações de $\Delta$}

No Passo 3.2, quando temos de escolher $\Delta \in\left[\Delta_{\min }, \Delta_{\text {bound }}\right)$, definimos

$$
\Delta=\max \left\{\Delta_{\min }, \Delta_{\min }+0.9\left(\left(\frac{\Delta_{\text {bound }}}{1+\sigma}\right)-\Delta_{\min }\right)\right\} .
$$

Isso porque, utilizando MEQB para encontrar o minimizador do modelo quadrático com raio $\Delta$, podemos ter o ponto $x^{\text {tent }}$ a uma distância $\sigma$ para fora da região de confiança. Utilizando a atualização (4.1) garantimos que, ao repetir o Passo 3.2, teremos o ponto $x^{\text {tent }}$ no interior da face.

No Passo 4, quando não obtemos decréscimo suficiente (ou seja, $\rho^{k}<0.1$ ), precisamos escolher $\Delta \in\left[0.1\left\|d^{k}\right\|, 0.9\left\|d^{k}\right\|\right.$. Neste caso, definimos

$$
\Delta=0.25\left\|d^{k}\right\| .
$$

No Passo 5, atualizamos o raio da região de confiança baseado na relação entre a redução obtida na função objetivo e a redução predita por seu modelo quadrático $\left(\rho^{k}\right)$ da seguinte maneira (veja [10]):

$$
\bar{\Delta}= \begin{cases}0.25\left\|d^{k}\right\|, & \text { if } \rho^{k} \leq 0.25 \\ 2 \Delta, & \text { if } \rho^{k} \geq 0.5 \text { e }\left|\left\|d^{k}\right\|-\Delta\right| \leq 10^{-5} \\ \Delta, & \text { caso contrário, }\end{cases}
$$

$\mathrm{e}$

$$
\Delta=\max \left\{\Delta_{\min }, \bar{\Delta}\right\}
$$

\subsubsection{Extrapolação}

Como dito anteriormente, utilizamos em nossa implementação do Algoritmo Interno a técnica de extrapolação apresentada em [2]. Veja como funciona esta técnica:

Extrapolação Dados $x^{k} \in A, d^{k}$ direção de descida, $\mu \in(0,1]$ e $N>1$

Passo 1. Decisão de extrapolar ou não

Se $\left(d^{k}\right)^{T} \nabla \bar{f}\left(x^{k}+d^{k}\right) \geq 0.5\left(d^{k}\right)^{T} \nabla \bar{f}\left(x^{k}\right)$ então

Termine a Extrapolação e devolva $x^{k}$

Passo 2. Calcule $\mu_{\max }=\max \left\{t \geq 0 \mid\left[x^{k}, x^{k}+t d^{k}\right] \subset A\right\}$

Passo 3. Cálculo do novo escalar que multiplica $d^{k}$

Se $\left(\mu<\mu_{\max }\right)$ e $\left(N \mu>\mu_{\max }\right)$ então 


$$
\mu_{\text {tent }}=\mu_{\max }
$$

Senão

$$
\mu_{\text {tent }}=N \mu
$$

Passo 4. Pára extrapolação quando ponto novo está muito perto do anterior

Se $\left(\mu \geq \mu_{\text {max }}\right)$ e $\left(\left\|P_{A}\left(x^{k}+\mu_{\text {tent }} d^{k}\right)-P_{A}\left(x^{k}+\mu d^{k}\right)\right\|_{\infty}<\right.$ $\left.\max \left\{\epsilon_{a b s}, \epsilon_{r e l}\left\|P_{A}\left(x^{k}+\mu d^{k}\right)\right\|_{\infty}\right\}\right)$ então

$x^{k+1}=P_{A}\left(x^{k}+\mu d^{k}\right)$

Termine a Extrapolação e devolva $x^{k+1}$

Passo 5. Pára extrapolação quando consegue decréscimo simples da função

Se $\bar{f}\left(P\left(x^{k}+\mu_{\text {tent }} d^{k}\right)\right) \geq \bar{f}\left(P\left(x^{k}+\mu d^{k}\right)\right)$ então

$x^{k+1}=P_{A}\left(x^{k}+\mu d^{k}\right)$

Termine a Extrapolação e devolva $x^{k+1}$

Senão

$\mu=\mu_{\text {tent }}$

Volte para o Passo 3

O parâmetro $\mu$ se refere ao escalar usado para calcular $y^{k}=x^{k}+\mu d^{k}$ no Algoritmo Interno. Note que podemos chegar ao Passo 6 do Algoritmo Interno, onde é feita a Extrapolação, saindo dos Passos 2, 3 ou 5. Assim, quando chegamos à Extrapolação vindos do Passo 2, temos que $\mu=t_{k}$; quando chegamos pelo Passo 3 , temos que $\mu=t_{\max }$; e, quando chegamos pelo Passo 5, temos $\mu=1$.

Em nossa implementação acrescentamos 3 parâmetros que permitem ao usuário escolher se a Extrapolação será ou não tentada a partir de cada um dos passos do Algoritmo Interno que levam a ela. Quando não se deseja tentar a Extrapolação, toma-se $x^{k+1}=y^{k}$ no Passo 6 do Algoritmo Interno. 


\section{Capítulo 5}

\section{Um método de restrições ativas para minimização em caixas}

Como já foi dito anteriormente, os métodos de restrições ativas para a minimização de funções com restrições de caixa seguem o seguinte princípio: se conhecemos quais restrições são satisfeitas por igualdade na solução (ativas) e quais não (inativas) podemos fixar algumas variáveis nos seus limitantes e resolver um problema irrestrito nas outras variáveis (variáveis livres). Por isso, os métodos de restrições ativas tentam, a cada iteração, inferir quais restrições serão ativas na solução e resolver um problema localmente irrestrito nas variáveis livres.

Recentemente, foi introduzido um novo método de restrições ativas para a minimização de problemas com restrições de caixa (veja [2]). Neste método, divide-se o conjunto factível $\Omega$ em faces abertas disjuntas. De acordo com um teste, decide-se se o algoritmo deve continuar a trabalhar na face corrente ou deve mudar de face. Utiliza-se um algoritmo interno para minimizar a função nas faces e o método do Gradiente Espectral Projetado para sair das faces. Neste capítulo apresentamos um método baseado naquele apresentado em [2], que utiliza o algoritmo proposto no capítulo 4 para trabalhar nas faces.

Na seção 5.1 apresentamos o método de restrições ativas para minimização em caixas. Na seção 5.2 apresentamos o teorema de convergência deste método. Na seção 5.3 apresentamos os detalhes de nossa implementação do método apresentado na seção 5.1 e, na seção 5.4 apresentamos os resultados numéricos obtidos por nossa implementação, bem como a comparação do desempenho de nosso método e de LANCELOT.

\subsection{O algoritmo}

Nesta seção,

$$
\Omega=\left\{x \in \mathbb{R}^{n} \mid \ell \leq x \leq u\right\}
$$

e $f: \mathbb{R}^{n} \rightarrow \mathbb{R}$ tem derivadas contínuas de terceira ordem num conjunto aberto que contém $\Omega$. Denotamos $g=\nabla f$. Como em [2], dividimos o conjunto factível $\Omega$ em faces abertas disjuntas. 
Para todo $I \subset\{1,2, \ldots, n, n+1, n+2, \ldots 2 n\}$ definimos

$$
F_{I}=\left\{x \in \Omega \mid x_{i}=\ell_{i} \text { se } i \in I, x_{i}=u_{i} \text { se } n+i \in I, \ell_{i}<x_{i}<u_{i} \text { caso contrário }\right\} .
$$

A caixa $\Omega$ é a união das faces abertas. As variáveis $x_{i}$ tais que nem $i$ nem $n+i$ pertencem a $I$ são chamadas livres em $F_{I}$. O Algoritmo Interno definido no capítulo 4 modifica somente as variáveis livres. Portanto, o conjunto convexo $A$ será identificado com os diferentes fechos das faces abertas. Definimos $V_{I}$ o menor espaço afim que contém $F_{I}$ e $S_{I}$ o subespaço linear paralelo a $V_{I}$. Definimos o gradiente projetado da seguinte maneira:

$$
g_{P}(x)=P_{\Omega}(x-g(x))-x
$$

$\mathrm{e}$, para todo $x \in F_{I}$, definimos o gradiente interno como

$$
g_{I}(x)=P_{S_{I}}\left[g_{P}(x)\right] .
$$

Observe que $g_{I}(x)=0$ quando a face $F_{I}$ é um vértice, pois, neste caso, $S_{I}=\{0\}$.

Como em [2], o algoritmo para minimização com restrições de caixa irá combinar iterações do Algoritmo Interno com iterações de SPG. Chamaremos de iterações do "SPG interno" às que iterações do SPG que forem feitas a partir do Passo 2 do Algoritmo Interno, e de iterações do "SPG global" àquelas que forem feitas a partir do Passo 4 do BETRA.

A idéia do método de restrições ativas é: dividimos o conjunto factível em faces abertas e, a cada iteração $k$, estamos num ponto $x^{k}$ pertencente a uma dessas faces. No início de cada iteração, verificamos qual a relação entre a norma do gradiente interno e a norma do gradiente projetado. Quando a norma do gradiente projetado é "muito maior" que a norma do gradiente interno, achamos que é interessante mudar de face e fazemos uma iteração do Gradiente Espectral Projetado (como mostrado no capítulo 3) para definir o novo ponto $x^{k+1}$, pertencente a uma nova face aberta. Quando a norma do gradiente projetado não é "muito maior", decidimos continuar na mesma face e utilizamos um iteração do Algoritmo Interno apresentado no capítulo 4 para obter um ponto $x^{k+1}$ onde a função tem um valor menor do que em $x^{k}$.

A seguir definimos o algoritmo de restrições ativas para minimização em caixas, chamado de BETRA (box-Euclidian-trust-region algorithm):

BETRA Dados $x^{0} \in \Omega$ e $\eta \in(0,1)$

Passo 1. $k=0$

Passo 2. Seja $F_{I}$ a face aberta à qual $x^{k}$ pertence.

$$
\text { Calcule } g_{P}\left(x^{k}\right) \text { e } g_{I}\left(x^{k}\right)
$$

Passo 3. Critério de convergência

Se $F_{I}$ é um vértice e $g_{P}\left(x^{k}\right)=0$ então 
Pare e devolva $x^{k}$ como solução

Passo 4. Decisão de permanecer ou não na mesma face

$\mathrm{Se}$

$$
\left\|g_{I}\left(x^{k}\right)\right\| \geq \eta\left\|g_{P}\left(x^{k}\right)\right\|
$$

Suponha, sem perda de generalidade, que as variáveis livres em $F_{I}$ são $x_{1}, \ldots, x_{m}$ e que as variáveis restantes estão fixas em $\bar{x}_{m+1}, \ldots, \bar{x}_{n}$ $\left(\bar{x}_{i} \in\left\{l_{i}, u_{i}\right\}\right.$, para $\left.m+1 \leq i \leq n\right)$. Defina $\bar{f}\left(x_{1}, \ldots, x_{m}\right)=f\left(x_{1}, \ldots, x_{m}, \bar{x}_{m+1}, \ldots, \bar{x}_{n}\right)$, para todo $x_{1}, \ldots, x_{m} \in \mathbb{R} \mathrm{e}$ $A=\left\{x \in \mathbb{R}^{m} \mid l_{i} \leq x_{i} \leq u_{i}, \forall i=1, \ldots, m\right\}$.

Use uma iteração do Algoritmo Interno para calcular $x^{k+1}$

Se o Algoritmo Interno terminar sua execução com $x^{k}$ declarando

"Ponto estacionário de primeira ordem perto da borda" ou

"Ponto estacionário de segunda ordem". então

Pare e devolva $x^{k}$ como solução,

declarando o mesmo que o Algoritmo Interno

Senão

Faça uma iteração do SPG $\operatorname{com} A=\Omega$ e tome $x^{k+1}=y^{k}$

Passo 5. $k=k+1$

Passo 6. Volte para o Passo 2

Note que, se a face corrente não é um vértice, o algoritmo BETRA não termina, necessariamente, quando, no início da iteração, $g_{P}\left(x^{k}\right)=0$. Neste caso, vale (5.1) e uma iteração do Algoritmo Interno é feita. Se $x^{k}$ não está perto da borda, um iteração de regiões de confiança é feita com o objetivo de encontrar um ponto estacionário de segunda ordem.

\subsection{Teorema de convergência}

Teorema 5.2.1 Vale uma das seguintes afirmações:

1. A execução do BETRA termina numa iteração onde o Algoritmo Interno termina com $g_{P}\left(x^{k}\right)=0$. Neste caso, se $x^{k} \in F_{I}$ e a distância entre $x^{k}$ e a borda de $F_{I}$ é maior que $2 \Delta_{\text {min }}$, a matriz $\nabla^{2} f\left(x^{k}\right)$ é semidefinida positiva quando restrita a $F_{I}$;

2. BETRA gera uma seqüência infinita de iterandos e pelo menos um ponto limite dessa seqüência é um ponto estacionário de primeira ordem. 
No segundo caso, se todos os pontos limite são não-degenerados, todos os iterandos pertencerão, em algum momento, à mesma face aberta $F_{I}$. Neste caso, se a distância de um ponto limite $x^{*} \dot{a}$ borda de $F_{I}$ for maior que $2 \Delta_{\text {min }}$, então $\nabla^{2} f\left(x^{*}\right)$ é semidefinida positiva quando restrita a $F_{I}$. Mais ainda, se esta matriz reduzida for definida positiva, a seqüência converge quadraticamente a $x^{*}$.

Prova Note que, pela definição de BETRA, a terminação finita ocorre somente quando $x^{k}$ é um vértice no qual $g_{P}\left(x^{k}\right)=0$ ou quando o Algoritmo Interno termina com as declarações "Ponto estacionário de primeira ordem perto da borda" ou "Ponto estacionário de segunda ordem". Em ambos os casos, temos que as derivadas correspondentes às variáveis livres são nulas, então $g_{I}\left(x^{k}\right)=0$. Então, por (5.1), temos que $g_{P}\left(x^{k}\right)=0$. Pelo Teorema 4.2.1, a hessiana reduzida é semidefinida positiva se a distância de $x^{k}$ até a borda da face à qual este ponto pertence é maior que $2 \Delta_{\min }$.

Falta agora considerar o caso em que BETRA gera infinitos iterandos. Consideremos duas possibilidades:

(a) Existe um conjunto infinito de índices $K_{1} \subset \mathbb{N}$ tal que $x^{k+1}$ é computado por uma iteração do SPG (global) para todo $k \in K_{1}$.

(b) Para todo $k \in \mathbb{I N}$ suficientemente grande, $x^{k+1}$ é computado pelo Algoritmo Interno.

Se vale (a), provamos, como em [2], que todo ponto limite de $\left\{x^{k}\right\}_{k \in K_{1}}$ é estacionário de primeira ordem restrito.

Se vale (b), existe $k_{0} \in \mathbb{N}$ tal que $x^{k+1}$ é computado pelo Algoritmo Interno para todo $k \geq k_{0}$. Como o número de faces é finito e restrições ativas são abandonadas apenas por iterações do SPG (global), existe uma face $F_{I}$ tal que $x^{k} \in F_{I}$ para todo $k$ suficientemente grande. Portanto, a tese do Teorema 4.2 .1 se aplica e, conseqüentemente, $\lim _{k \rightarrow \infty} g_{I}\left(x^{k}\right)=0$. Então, por (5.1), $\lim _{k \rightarrow \infty} g_{P}\left(x^{k}\right)=0$. Isso implica que todos os pontos limites satisfazem $g_{P}\left(x^{*}\right)=0$. Ainda pelo Teorema 4.2.1, se a distância de um ponto limite até a borda for maior que $2 \Delta_{\min }$, a hessiana reduzida é semidefinida positiva. Neste caso, se a hessiana for definida positiva, a convergência quadrática segue como na teoria clássica do método newtoniano irrestrito de regiões de confiança (veja [22]). O fato de que no caso de nãodegenerescência todos os iterandos pertencem, em algum momento, à mesma face segue como no Teorema 3.4 de [1].

\subsection{Implementação}

Implementamos o algoritmo BETRA em FORTRAN 77 para comparar seu comportamento com LANCELOT, outro método para minimização de funções com restrições de caixa (veja $[8])$. 
Para calcular o raio inicial da região de confiança, recebemos um parâmetro $\Delta_{\text {inicial }}$ e calculamos $\Delta$ da seguinte forma:

$$
\Delta=\max \left\{\Delta_{\text {min }}, \Delta_{\text {inicial }} \max \left\{1,\left\|x^{0}\right\|\right\}\right\}
$$

BETRA possui 4 critérios de parada:

1. Vértice estacionário de primeira ordem (Passo 3 do BETRA):

A face corrente é um vértice e $\left\|g_{P}\left(x^{k}\right)\right\|_{\infty} \leq \varepsilon ;$

2. Ponto estacionário de primeira ordem perto da borda (Passo 2 do Algoritmo Interno): Valem as desigualdades $\Delta_{b o r d a}<2 \Delta_{\min }$ e $\left\|\bar{g}\left(x^{k}\right)\right\|_{\infty} \leq \varepsilon ;$

3. Ponto estacionário de segunda ordem (Passo 3 do Algoritmo Interno):

$d^{k}$ é solução do subproblema de regiões de confiança, $\left|\varphi_{k}\left(d^{k}\right)\right|<\varepsilon$ e $\left\|\bar{g}\left(x^{k}\right)\right\|_{\infty}<\varepsilon$;

4. Ponto estacionário de primeira ordem (Passo 4 do BETRA):

O teste (5.1) não vale (a face corrente deve ser abandonada) e $\left\|g_{P}\left(x^{k}\right)\right\|_{\infty}<\varepsilon$.

Nos experimentos utilizamos $\epsilon=10^{-5}$ para MEQB; $\alpha=10^{-4}, \lambda_{\min }^{\mathrm{SPG}}=10^{-10}, \lambda_{\max }^{\mathrm{SPG}}=10^{10}$, $\epsilon_{\text {rel }}=10^{-7}$ e $\epsilon_{a b s}=10^{-10}$ para SPG; $\varepsilon=10^{-5}$ para o critério de parada (para o teste (5.1) utilizamos norma infinito).

Para decidir os parâmetros restantes $\left(\eta, \Delta_{\text {inicial }}, \Delta_{\min }, \sigma\right.$ e $\left.N\right)$ testamos todas as combinações de $\eta \in\{0.1,0.9\}, \Delta_{\text {inicial }} \in\{0.1,1,10,100\}, \Delta_{\min } \in\left\{10^{-5}, 10^{-4}, 10^{-3}\right\}, \sigma \in$ $\{0.1,0.2\}$, e $N \in\{2,4\}$. A combinação que forneceu os melhores resultados foi $\eta=0.1$, $\Delta_{\text {inicial }}=100, \Delta_{\min }=10^{-4}, \sigma=0.2$, e $N=4$. Extrapolação só é permitida quando o Passo 6 do Algoritmo Interno é atingida pelos Passos 3 e 5 . Levamos em consideração a robustez do BETRA para a escolha desses parâmetros.

\subsection{Resultados numéricos}

Para comparar o desempenho do BETRA e do LANCELOT, especificamos que este deveria usar segunda derivada exata e a opção "full-matrix-preconditioned-cg-solver-used" fosse ativada (para forçar que o LANCELOT decomponha a hessiana, como faz o BETRA). Para fazer tal comparação, utilizamos os problemas da coleção CUTE (Constrained and Unconstrained Testing Environment, veja [6]) com restrições de caixa e dimensões menores ou iguais a 120, totalizando 140 problemas. Como os tempos de execução de ambos os métodos para os 140 problemas selecionados são, em geral, muito pequenos, executamos cada par método/problema várias vezes, a fim de evitar erros na medição do tempo. 
Todos os experimentos foram feitos utilizando o compilador g77 - GNU project Fortran Compiler (v0.5.24), com opção de compilação -O3 para otimizar o código. Usando um Pentium I com $133 \mathrm{MHZ}, 64 \mathrm{Mb}$ de memória RAM, com sistema operacional Linux.

Nas tabelas 5.1, 5.2 e 5.3 apresentamos o desempenho de BETRA e LANCELOT em todo o conjunto de problemas. Nas tabelas, temos nas colunas:

Problema (n): nome do problema e o número de variáveis;

naf: número de avaliações de função;

nag: número de avaliações de gradiente;

nah: número de avaliações de hessiana;

nch: número de fatorações de Cholesky;

ncg: número de iterações de Gradientes Conjugados;

Tempo: tempo de CPU em segundos;

$f\left(x^{*}\right)$ : valor da função na melhor solução encontrada.

O número de avaliações de hessiana feitas pelo LANCELOT é nag - 1. As linhas duplas dividem os problemas de acordo com o tipo da função objetivo. As funções objetivo dos problemas do primeiro grupo são quadráticas, as do segundo grupo são soma de quadrados, e as do terceiro grupo são não-lineares.

Em média, $12.48 \%$ das avaliações de função foram feitas pelo SPG global, $3.87 \%$ foram feitas pelo SPG interno e $83.65 \%$ foram feitas por iterações de regiões de confiança do Algoritmo Interno. Dos 140 problemas testados, 33 problemas $(23.57 \%)$ satisfizeram o critério 1, 5 problemas $(3.57 \%)$ satisfizeram o critério 2, 98 (70.71\%) satisfizeram o critério 3, e nenhum satisfez o critério 4. Os 3 problemas restantes pararam porque BETRA atingiu o número máximo de iterações permitidas (10000). Estes problemas foram: PALMER5A que parou com $\left\|g_{P}\left(x^{k}\right)\right\|_{\infty}=1.8088 \mathrm{E}+00$; PALMER7A, que parou com $\left\|g_{P}\left(x^{k}\right)\right\|_{\infty}=4.0094 \mathrm{E}-01$; e SINEALI (20) que parou com $\left\|g_{P}\left(x^{k}\right)\right\|_{\infty}=2.6944 \mathrm{E}-03$.

LANCELOT possui apenas um critério de parada com sucesso: a norma infinito do gradiente projetado deve menor que $10^{-5}$. Em 137 dos 140 problemas testados, este critério foi satisfeito. Nos 3 problemas restantes, LANCELOT parou porque atingiu o número máximo de avaliações de função permitidas (10000). Estes problemas foram: PALMER5A que parou com $\left\|g_{P}\left(x^{k}\right)\right\|_{\infty}=1.5959 \mathrm{E}-01$; PALMER7E que parou com $\left\|g_{P}\left(x^{k}\right)\right\|_{\infty}=5.9967 \mathrm{E}+00$; e SINEALI (20) que parou com $\left\|g_{P}\left(x^{k}\right)\right\|_{\infty}=7.9770 \mathrm{E}-04$. É importante ressaltar que, apesar de BETRA possuir maior número de critérios de parada, os critérios diferentes daquele adotado por LANCELOT são mais fortes. Assim, BETRA não pode parar "antes" de LANCELOT e prejudicar a comparação entre ambos.

Para visualizar melhor a comparação do desempenho dos dois métodos, utilizamos o tempo de CPU gasto pelos métodos para resolver os problemas como fator de comparação e construímos um gráfico do perfil de desempenho (veja [9]). Neste gráfico, se um ponto da curva correspondente ao método $M$ vale $I$ no eixo das abscissas e $J$ no eixo das ordenadas, significa que o método $M$ é o $I$-ésimo método mais rápido em $(J \times 100) \%$ dos problemas. Os pontos mais significativos do gráfico são o mais à esquerda e o mais à direita. À esquerda medimos a eficiência dos métodos (verificamos a porcentagem de problemas para os quais cada método 


\begin{tabular}{|c|c|c|c|c|c|c|c|c|c|c|c|}
\hline & \multicolumn{6}{|c|}{ BETRA } & \multicolumn{5}{|c|}{ LANCELOT } \\
\hline Problema (n) & naf & nag & nah & nch & Tempo & $f\left(x^{*}\right)$ & naf & nag & ncg & Tempo & $f\left(x^{*}\right)$ \\
\hline BQP1VAR (1) & 2 & 2 & 1 & 1 & 0.000 & $0.0000 \mathrm{E}+00$ & 3 & 3 & 0 & 0.004 & $0.0000 \mathrm{E}+00$ \\
\hline HS3 (2) & 3 & 4 & 3 & 4 & 0.001 & $7.8886 \mathrm{E}-36$ & 8 & 8 & 0 & 0.009 & $9.5614 \mathrm{E}-37$ \\
\hline HS3MOD (2) & 3 & 4 & 3 & 5 & 0.001 & $7.8886 \mathrm{E}-31$ & 4 & 4 & 3 & 0.008 & $4.1591 \mathrm{E}-32$ \\
\hline OSLBQP (8) & 2 & 2 & 2 & 2 & 0.001 & $6.2500 \mathrm{E}+00$ & 3 & 3 & 0 & 0.008 & $6.2500 \mathrm{E}+00$ \\
\hline SIM2BQP (2) & 2 & 3 & 0 & 0 & 0.000 & $0.0000 \mathrm{E}+00$ & 3 & 3 & 0 & 0.005 & $0.0000 \mathrm{E}+00$ \\
\hline SIMBQP (2) & 5 & 5 & 4 & 4 & 0.001 & $2.4074 \mathrm{E}-34$ & 2 & 2 & 0 & 0.005 & $1.1132 \mathrm{E}-30$ \\
\hline HATFLDA (4) & 21 & 14 & 13 & 16 & 0.005 & $1.8367 \mathrm{E}-14$ & 28 & 28 & 22 & 0.037 & $2.7495 \mathrm{E}-14$ \\
\hline HATFLDB (4) & 18 & 11 & 10 & 13 & 0.004 & $5.5728 \mathrm{E}-03$ & 25 & 25 & 20 & 0.033 & $5.5728 \mathrm{E}-03$ \\
\hline HS1 (2) & 29 & 27 & 25 & 39 & 0.005 & $4.3945 \mathrm{E}-15$ & 33 & 28 & 23 & 0.033 & $1.2242 \mathrm{E}-12$ \\
\hline HS2 (2) & 6 & 6 & 6 & 6 & 0.001 & $4.9412 \mathrm{E}+00$ & 7 & 7 & 2 & 0.010 & $4.9412 \mathrm{E}+00$ \\
\hline HS25 (3) & 1 & 1 & 1 & 1 & 0.013 & $3.2835 \mathrm{E}+01$ & 1 & 1 & 0 & 0.013 & $3.2835 \mathrm{E}+01$ \\
\hline PALMER1 (4) & 34 & 38 & 28 & 67 & 0.069 & $1.1755 \mathrm{E}+04$ & 26 & 22 & 14 & 0.080 & $1.1755 \mathrm{E}+04$ \\
\hline PALMERIA (6) & 87 & 99 & 80 & 136 & 0.255 & $8.9883 \mathrm{E}-02$ & 78 & 68 & 61 & 0.308 & $8.9883 \mathrm{E}-02$ \\
\hline PALMER1B (4) & 79 & 100 & 71 & 116 & 0.165 & $3.4473 \mathrm{E}+00$ & 44 & 38 & 25 & 0.119 & $3.4473 \mathrm{E}+00$ \\
\hline PALMER1E (8) & 102 & 105 & 80 & 132 & 0.441 & $8.3523 \mathrm{E}-04$ & 5 & 5 & 5 & 0.034 & $3.1103 \mathrm{E}+00$ \\
\hline PALMER2 (4) & 21 & 23 & 16 & 34 & 0.033 & $3.6511 \mathrm{E}+03$ & 28 & 23 & 12 & 0.068 & $3.6511 \mathrm{E}+03$ \\
\hline PALMER2A (6) & 50 & 55 & 47 & 84 & 0.109 & $1.7110 \mathrm{E}-02$ & 100 & 86 & 94 & 0.316 & $1.7110 \mathrm{E}-02$ \\
\hline PALMER2B (4) & 58 & 71 & 51 & 95 & 0.081 & $6.2327 \mathrm{E}-01$ & 25 & 22 & 15 & 0.060 & $6.2327 \mathrm{E}-01$ \\
\hline PALMER2E (8) & 81 & 89 & 76 & 116 & 0.282 & $2.0650 \mathrm{E}-04$ & 315 & 261 & 320 & 1.357 & $2.0650 \mathrm{E}-04$ \\
\hline PALMER3A (6) & 95 & 116 & 89 & 143 & 0.209 & $2.0431 \mathrm{E}-02$ & 89 & 76 & 86 & 0.286 & $2.0431 \mathrm{E}-02$ \\
\hline PALMER3B (4) & 47 & 63 & 41 & 67 & 0.068 & $4.2276 \mathrm{E}+00$ & 28 & 23 & 22 & 0.067 & $4.2276 \mathrm{E}+00$ \\
\hline PALMER3E (8) & 53 & 58 & 48 & 92 & 0.182 & $5.0741 \mathrm{E}-05$ & 57 & 47 & 57 & 0.251 & $5.0741 \mathrm{E}-05$ \\
\hline PALMER4 (4) & 24 & 22 & 16 & 33 & 0.030 & $2.2854 \mathrm{E}+03$ & 47 & 38 & 22 & 0.117 & $2.2854 \mathrm{E}+03$ \\
\hline PALMER4A (6) & 42 & 41 & 38 & 82 & 0.085 & $4.0606 \mathrm{E}-02$ & 67 & 56 & 65 & 0.211 & $4.0606 \mathrm{E}-02$ \\
\hline PALMER4B (4) & 55 & 72 & 50 & 84 & 0.086 & $6.8351 \mathrm{E}+00$ & 26 & 22 & 18 & 0.063 & $6.8351 \mathrm{E}+00$ \\
\hline PALMER4E (8) & 50 & 51 & 48 & 80 & 0.171 & $1.4800 \mathrm{E}-04$ & 40 & 32 & 40 & 0.176 & $1.4800 \mathrm{E}-04$ \\
\hline PALMER5A (8) & 10029 & 15633 & 10000 & 10474 & 20.280 & $4.0560 \mathrm{E}-02$ & 10001 & 8402 & 9975 & 27.250 & $3.1022 \mathrm{E}-02$ \\
\hline PALMER5B (9) & 646 & 872 & 627 & 886 & 1.326 & $9.7524 \mathrm{E}-03$ & 123 & 101 & 114 & 0.377 & $9.7524 \mathrm{E}-03$ \\
\hline PALMER5D (8) & 3 & 3 & 3 & 5 & 0.002 & $8.7339 \mathrm{E}+01$ & 2 & 2 & 1 & 0.010 & $8.7339 \mathrm{E}+01$ \\
\hline PALMER5E (8) & 1437 & 1989 & 1428 & 1511 & 3.015 & $2.2937 \mathrm{E}-02$ & 8763 & 7212 & 8762 & 24.970 & $2.0716 \mathrm{E}-02$ \\
\hline PALMER6A (6) & 124 & 143 & 117 & 188 & 0.171 & $5.5949 \mathrm{E}-02$ & 141 & 120 & 134 & 0.317 & $5.5949 \mathrm{E}-02$ \\
\hline PALMER6E (8) & 82 & 101 & 80 & 111 & 0.185 & $2.2395 \mathrm{E}-04$ & 61 & 50 & 62 & 0.189 & $2.2395 \mathrm{E}-04$ \\
\hline PALMER7A (6) & 10008 & 15505 & 10000 & 10116 & 15.220 & $1.0345 \mathrm{E}+01$ & 4007 & 3569 & 4008 & 8.910 & $1.0335 \mathrm{E}+01$ \\
\hline PALMER7E (8) & 6 & 7 & 6 & 17 & 0.013 & $1.0154 \mathrm{E}+01$ & 10001 & 8065 & 9930 & 29.120 & $6.5607 \mathrm{E}+00$ \\
\hline PALMER8A (6) & 38 & 40 & 36 & 68 & 0.049 & $7.4010 \mathrm{E}-02$ & 52 & 45 & 55 & 0.119 & $7.4010 \mathrm{E}-02$ \\
\hline PALMER8E (8) & 27 & 37 & 26 & 52 & 0.058 & $6.3393 \mathrm{E}-03$ & 41 & 34 & 41 & 0.124 & $6.3393 \mathrm{E}-03$ \\
\hline PSPDOC (4) & 8 & 6 & 6 & 11 & 0.002 & $2.4142 \mathrm{E}+00$ & 10 & 10 & 9 & 0.015 & $2.4142 \mathrm{E}+00$ \\
\hline WEEDS (3) & 23 & 8 & 5 & 10 & 0.007 & $9.2054 \mathrm{E}+03$ & 25 & 22 & 27 & 0.050 & $2.5873 \mathrm{E}+00$ \\
\hline YFIT (3) & 60 & 63 & 50 & 93 & 0.086 & $6.6697 \mathrm{E}-13$ & 51 & 42 & 50 & 0.112 & $6.6698 \mathrm{E}-13$ \\
\hline ALLINIT (4) & 12 & 11 & 10 & 13 & 0.007 & $1.6706 \mathrm{E}+01$ & 12 & 10 & 6 & 0.017 & $1.6706 \mathrm{E}+01$ \\
\hline CAMEL6 (2) & 37 & 30 & 30 & 49 & 0.010 & $-1.0316 \mathrm{E}+00$ & 19 & 11 & 12 & 0.020 & $-1.0316 \mathrm{E}+00$ \\
\hline HART6 (6) & 15 & 16 & 13 & 22 & 0.013 & $-3.3229 \mathrm{E}+00$ & 9 & 7 & 7 & 0.019 & $-3.3229 \mathrm{E}+00$ \\
\hline HIMMELP1 (2) & 9 & 7 & 6 & 8 & 0.002 & $-6.2054 \mathrm{E}+01$ & 16 & 15 & 5 & 0.014 & $-6.2054 \mathrm{E}+01$ \\
\hline HS38 (4) & 55 & 54 & 49 & 91 & 0.023 & $1.9971 \mathrm{E}-20$ & 54 & 46 & 56 & 0.069 & $5.2378 \mathrm{E}-20$ \\
\hline HS4 (2) & 2 & 3 & 1 & 2 & 0.000 & $2.6667 \mathrm{E}+00$ & 2 & 2 & 0 & 0.004 & $2.6667 \mathrm{E}+00$ \\
\hline HS45 (5) & 2 & 3 & 1 & 13 & 0.001 & $1.0000 \mathrm{E}+00$ & 9 & 9 & 0 & 0.009 & $1.0000 \mathrm{E}+00$ \\
\hline HS5 (2) & 10 & 9 & 8 & 16 & 0.002 & $-1.9132 \mathrm{E}+00$ & 6 & 6 & 3 & 0.009 & $-1.9132 \mathrm{E}+00$ \\
\hline LOGROS (2) & 73 & 59 & 54 & 127 & 0.018 & $0.0000 \mathrm{E}+00$ & 56 & 45 & 35 & 0.054 & $0.0000 \mathrm{E}+00$ \\
\hline MAXLIKA (8) & 56 & 56 & 35 & 61 & 4.017 & $1.1363 \mathrm{E}+03$ & 9 & 8 & 19 & 0.659 & $1.1493 \mathrm{E}+03$ \\
\hline MDHOLE (2) & 39 & 43 & 37 & 65 & 0.010 & $4.8148 \mathrm{E}-33$ & 61 & 51 & 52 & 0.058 & $3.0080 \mathrm{E}-37$ \\
\hline S368 (8) & 6 & 9 & 6 & 8 & 0.026 & $-9.3750 \mathrm{E}-01$ & 6 & 6 & 3 & 0.029 & $-9.3750 \mathrm{E}-01$ \\
\hline
\end{tabular}

Tabela 5.1: Desempenho de BETRA e LANCELOT nos problemas com menos de 10 variáveis. 


\begin{tabular}{|c|c|c|c|c|c|c|c|c|c|c|c|}
\hline & \multicolumn{6}{|c|}{ BETRA } & \multicolumn{5}{|c|}{ LANCELOT } \\
\hline Problema (n) & naf & nag & nah & nch & Tempo & $f\left(x^{*}\right)$ & naf & nag & ncg & Tempo & $f\left(x^{*}\right)$ \\
\hline BIGGSB1 (25) & 42 & 28 & 15 & 15 & 0.026 & $1.5000 \mathrm{E}-02$ & 15 & 15 & 14 & 0.041 & $1.5000 \mathrm{E}-02$ \\
\hline BQPGABIM (50) & 60 & 46 & 0 & 0 & 0.053 & $-3.7903 \mathrm{E}-05$ & 3 & 3 & 4 & 0.042 & $-3.7903 \mathrm{E}-05$ \\
\hline BQPGASIM (50) & 56 & 45 & 0 & 0 & 0.054 & $-5.5198 \mathrm{E}-05$ & 3 & 3 & 3 & 0.040 & $-5.5198 \mathrm{E}-05$ \\
\hline CHENHARK (10) & 8 & 8 & 5 & 5 & 0.004 & $-2.0000 \mathrm{E}+00$ & 2 & 2 & 5 & 0.011 & $-2.0000 \mathrm{E}+00$ \\
\hline CVXBQP1 (10) & 3 & 3 & 1 & 3 & 0.001 & $2.4750 \mathrm{E}+00$ & 2 & 2 & 0 & 0.009 & $2.4750 \mathrm{E}+00$ \\
\hline HARKERP2 (10) & 9 & 11 & 9 & 9 & 0.011 & $-5.0000 \mathrm{E}-01$ & 3 & 3 & 1 & 0.011 & $-5.0000 \mathrm{E}-01$ \\
\hline JNLBRNG1 (16) & 2 & 2 & 2 & 2 & 0.002 & $-2.2474 \mathrm{E}-01$ & 2 & 2 & 2 & 0.012 & $-2.2474 \mathrm{E}-01$ \\
\hline JNLBRNG2 (16) & 2 & 2 & 2 & 2 & 0.002 & $-4.7640 \mathrm{E}+00$ & 2 & 2 & 0 & 0.011 & $-4.7640 \mathrm{E}+00$ \\
\hline JNLBRNGA (16) & 3 & 4 & 2 & 2 & 0.002 & $-5.0967 \mathrm{E}-01$ & 5 & 5 & 0 & 0.013 & $-5.0967 \mathrm{E}-01$ \\
\hline JNLBRNGB (16) & 3 & 4 & 2 & 2 & 0.002 & $-1.8551 \mathrm{E}+01$ & 5 & 5 & 1 & 0.013 & $-1.8551 \mathrm{E}+01$ \\
\hline NCVXBQP1 (10) & 2 & 3 & 1 & 1 & 0.001 & $-2.2050 \mathrm{E}+04$ & 3 & 3 & 0 & 0.010 & $-2.2050 \mathrm{E}+04$ \\
\hline NCVXBQP2 (10) & 2 & 3 & 1 & 1 & 0.001 & $-1.4382 \mathrm{E}+04$ & 3 & 3 & 0 & 0.010 & $-1.4382 \mathrm{E}+04$ \\
\hline NCVXBQP3 (10) & 3 & 4 & 1 & 1 & 0.001 & $-1.1958 \mathrm{E}+04$ & 3 & 3 & 0 & 0.010 & $-1.1958 \mathrm{E}+04$ \\
\hline NOBNDTOR (16) & 2 & 2 & 2 & 2 & 0.001 & $-5.4321 \mathrm{E}-01$ & 3 & 3 & 0 & 0.011 & $-5.4321 \mathrm{E}-01$ \\
\hline OBSTCLAE (16) & 2 & 3 & 1 & 1 & 0.001 & $7.5366 \mathrm{E}-01$ & 3 & 3 & 0 & 0.010 & $7.5366 \mathrm{E}-01$ \\
\hline OBSTCLAL (16) & 1 & 1 & 0 & 0 & 0.000 & $7.5366 \mathrm{E}-01$ & 1 & 1 & 0 & 0.008 & $7.5366 \mathrm{E}-01$ \\
\hline OBSTCLBL (16) & 2 & 3 & 0 & 0 & 0.000 & $-8.1108 \mathrm{E}-03$ & 2 & 2 & 0 & 0.009 & $-8.1108 \mathrm{E}-03$ \\
\hline OBSTCLBM (16) & 2 & 3 & 1 & 1 & 0.001 & $-8.1108 \mathrm{E}-03$ & 2 & 2 & 0 & 0.010 & $-8.1108 \mathrm{E}-03$ \\
\hline OBSTCLBU (16) & 1 & 1 & 0 & 0 & 0.000 & $-8.1108 \mathrm{E}-03$ & 1 & 1 & 0 & 0.009 & $-8.1108 \mathrm{E}-03$ \\
\hline PENTDI (50) & 3 & 4 & 2 & 2 & 0.007 & $-7.5000 \mathrm{E}-01$ & 2 & 2 & 0 & 0.023 & $-7.5000 \mathrm{E}-01$ \\
\hline QUDLIN (12) & 2 & 3 & 0 & 0 & 0.000 & $-7.2000 \mathrm{E}+03$ & 2 & 2 & 0 & 0.011 & $-7.2000 \mathrm{E}+03$ \\
\hline TORSION1 (16) & 1 & 1 & 0 & 0 & 0.000 & $-5.1852 \mathrm{E}-01$ & 1 & 1 & 0 & 0.009 & $-5.1852 \mathrm{E}-01$ \\
\hline TORSION2 (16) & 2 & 2 & 1 & 1 & 0.001 & $-5.1852 \mathrm{E}-01$ & 3 & 3 & 0 & 0.010 & $-5.1852 \mathrm{E}-01$ \\
\hline TORSION3 (16) & 1 & 1 & 0 & 0 & 0.000 & $-1.2593 \mathrm{E}+00$ & 1 & 1 & 0 & 0.008 & $-1.2593 \mathrm{E}+00$ \\
\hline TORSION4 (16) & 2 & 2 & 1 & 1 & 0.001 & $-1.2593 \mathrm{E}+00$ & 3 & 3 & 0 & 0.010 & $-1.2593 \mathrm{E}+00$ \\
\hline TORSION5 (16) & 1 & 1 & 0 & 0 & 0.000 & $-2.7407 \mathrm{E}+00$ & 1 & 1 & 0 & 0.008 & $-2.7407 \mathrm{E}+00$ \\
\hline TORSION6 (16) & 2 & 2 & 1 & 1 & 0.001 & $-2.7407 \mathrm{E}+00$ & 2 & 2 & 0 & 0.009 & $-2.7407 \mathrm{E}+00$ \\
\hline TORSIONA (16) & 3 & 4 & 2 & 2 & 0.003 & $-3.0864 \mathrm{E}-01$ & 2 & 2 & 0 & 0.011 & $-3.0864 \mathrm{E}-01$ \\
\hline TORSIONB (16) & 2 & 2 & 2 & 2 & 0.003 & $-3.0864 \mathrm{E}-01$ & 3 & 3 & 0 & 0.012 & $-3.0864 \mathrm{E}-01$ \\
\hline TORSIONC (16) & 1 & 1 & 0 & 0 & 0.000 & $-1.0370 \mathrm{E}+00$ & 1 & 1 & 0 & 0.008 & $-1.0370 \mathrm{E}+00$ \\
\hline TORSIOND (16) & 2 & 2 & 1 & 1 & 0.002 & $-1.0370 \mathrm{E}+00$ & 3 & 3 & 0 & 0.011 & $-1.0370 \mathrm{E}+00$ \\
\hline TORSIONE (16) & 1 & 1 & 0 & 0 & 0.000 & $-2.5185 \mathrm{E}+00$ & 1 & 1 & 0 & 0.009 & $-2.5185 \mathrm{E}+00$ \\
\hline TORSIONF (16) & 2 & 2 & 1 & 1 & 0.002 & $-2.5185 \mathrm{E}+00$ & 2 & 2 & 0 & 0.010 & $-2.5185 \mathrm{E}+00$ \\
\hline CHEBYQAD (50) & 27 & 24 & 22 & 103 & 11.300 & $5.3863 \mathrm{E}-03$ & 106 & 87 & 108 & 43.310 & $5.3863 \mathrm{E}-03$ \\
\hline DECONVB (61) & 12006 & 7809 & 22 & 52 & 29.894 & $5.3548 \mathrm{E}-08$ & 20 & 16 & 128 & 0.852 & $5.6636 \mathrm{E}-03$ \\
\hline HATFLDC (25) & 5 & 5 & 5 & 5 & 0.013 & $8.2639 \mathrm{E}-14$ & 5 & 5 & 3 & 0.026 & $7.7700 \mathrm{E}-19$ \\
\hline HS110 (10) & 7 & 6 & 6 & 8 & 0.009 & $-4.5778 \mathrm{E}+01$ & 5 & 5 & 0 & 0.016 & $-4.5778 \mathrm{E}+01$ \\
\hline LINVERSE (19) & 6 & 6 & 6 & 9 & 0.017 & $7.0000 \mathrm{E}+00$ & 10 & 8 & 16 & 0.055 & $7.0000 \mathrm{E}+00$ \\
\hline NONSCOMP (50) & 39 & 23 & 7 & 7 & 0.078 & $1.1916 \mathrm{E}-11$ & 9 & 9 & 8 & 0.065 & $1.1212 \mathrm{E}-16$ \\
\hline QR3DLS (40) & 23 & 21 & 20 & 43 & 0.324 & $5.5383 \mathrm{E}-16$ & 29 & 24 & 28 & 0.477 & $1.2807 \mathrm{E}-13$ \\
\hline EXPLIN (12) & 11 & 12 & $\overline{99}$ & 14 & 0.006 & $-6.8500 \mathrm{E}+03$ & 13 & 11 & 13 & 0.024 & $-6.8500 \mathrm{E}+03$ \\
\hline EXPLIN2 (12) & 13 & 14 & 9 & 16 & 0.007 & $-7.0925 \mathrm{E}+03$ & 14 & 13 & 16 & 0.027 & $-7.0925 \mathrm{E}+03$ \\
\hline EXPQUAD (12) & 11 & 14 & 10 & 45 & 0.019 & $-4.2011 \mathrm{E}+03$ & 13 & 11 & 20 & 0.033 & $-4.2011 \mathrm{E}+03$ \\
\hline HADAMALS (16) & 17 & 20 & 16 & 22 & 0.045 & $0.0000 \mathrm{E}+00$ & 8 & 8 & 37 & 0.046 & $0.0000 \mathrm{E}+00$ \\
\hline MCCORMCK (10) & 7 & 7 & 7 & 11 & 0.008 & $-9.5980 \mathrm{E}+00$ & 5 & 5 & 4 & 0.016 & $-9.5980 \mathrm{E}+00$ \\
\hline PROBPENL (10) & 34 & 25 & 19 & 62 & 0.030 & $-3.1787 \mathrm{E}+05$ & 5 & 3 & 13 & 0.020 & $1.5235 \mathrm{E}-05$ \\
\hline QRTQUAD (12) & 35 & 33 & 20 & 30 & 0.020 & $-3.6077 \mathrm{E}+03$ & 71 & 56 & 64 & 0.117 & $-3.6077 \mathrm{E}+03$ \\
\hline SINEALI $(20)$ & 10221 & 10626 & 10000 & 11627 & 23.720 & $-1.9010 \mathrm{E}+03$ & 10001 & 8887 & 9994 & 26.070 & $-1.9010 \mathrm{E}+03$ \\
\hline
\end{tabular}

Tabela 5.2: Desempenho de BETRA e LANCELOT nos problemas com mais de 10 e menos de 100 variáveis. 


\begin{tabular}{|c|c|c|c|c|c|c|c|c|c|c|c|}
\hline & \multicolumn{6}{|c|}{ BETRA } & \multicolumn{5}{|c|}{ LANCELOT } \\
\hline Problema (n) & naf & nag & nah & nch & Tempo & $f\left(x^{*}\right)$ & naf & nag & ncg & Tempo & $f\left(x^{*}\right)$ \\
\hline BIGGSB1 (100) & 284 & 103 & 52 & 52 & 0.950 & $1.5000 \mathrm{E}-02$ & 52 & 52 & 50 & 0.323 & $1.5000 \mathrm{E}-02$ \\
\hline CHENHARK (100) & 3 & 4 & 3 & 6 & 0.145 & $-2.0000 \mathrm{E}+00$ & 25 & 25 & 61 & 0.279 & $-2.0000 \mathrm{E}+00$ \\
\hline CVXBQP1 (100) & 3 & 3 & 1 & 3 & 0.104 & $2.2725 \mathrm{E}+02$ & 2 & 2 & 0 & 0.055 & $2.2725 \mathrm{E}+02$ \\
\hline HARKERP2 (100) & 7 & 9 & 7 & 7 & 12.570 & $-5.0000 \mathrm{E}-01$ & 2 & 2 & 2 & 0.223 & $-5.0000 \mathrm{E}-01$ \\
\hline JNLBRNG1 (100) & 5 & 4 & 3 & 3 & 0.050 & $-1.7896 \mathrm{E}-01$ & 2 & 2 & 1 & 0.056 & $-1.7896 \mathrm{E}-01$ \\
\hline JNLBRNG2 (100) & 4 & 4 & 3 & 3 & 0.046 & $-3.9528 \mathrm{E}+00$ & 3 & 3 & 2 & 0.066 & $-3.9528 \mathrm{E}+00$ \\
\hline JNLBRNGA (100) & 6 & 6 & 3 & 3 & 0.044 & $-3.6116 \mathrm{E}-01$ & 3 & 3 & 2 & 0.061 & $-3.6116 \mathrm{E}-01$ \\
\hline JNLBRNGB (100) & 3 & 4 & 2 & 2 & 0.026 & $-7.2552 \mathrm{E}+00$ & 4 & 4 & 3 & 0.069 & $-7.2552 \mathrm{E}+00$ \\
\hline NCVXBQP1 (100) & 2 & 3 & 1 & 1 & 0.045 & $-1.9956 \mathrm{E}+06$ & 2 & 2 & 0 & 0.057 & $-1.9956 \mathrm{E}+06$ \\
\hline NCVXBQP2 (100) & 6 & 7 & 3 & 6 & 0.058 & $-1.3330 \mathrm{E}+06$ & 3 & 3 & 4 & 0.061 & $-1.3330 \mathrm{E}+06$ \\
\hline NCVXBQP3 (100) & 7 & 9 & 5 & 6 & 0.066 & $-6.7085 \mathrm{E}+05$ & 4 & 4 & 4 & 0.064 & $-6.7085 \mathrm{E}+05$ \\
\hline NOBNDTOR (100) & 8 & 6 & 4 & 4 & 0.060 & $-5.5211 \mathrm{E}-01$ & 3 & 3 & 2 & 0.067 & $-5.5211 \mathrm{E}-01$ \\
\hline OBSTCLAE (100) & 9 & 9 & 5 & 5 & 0.079 & $1.3979 \mathrm{E}+00$ & 3 & 3 & 29 & 0.138 & $1.3979 \mathrm{E}+00$ \\
\hline OBSTCLAL (100) & 9 & 8 & 4 & 4 & 0.060 & $1.3979 \mathrm{E}+00$ & 4 & 4 & 3 & 0.071 & $1.3979 \mathrm{E}+00$ \\
\hline OBSTCLBL (100) & 7 & 10 & 5 & 5 & 0.056 & $2.8750 \mathrm{E}+00$ & 3 & 3 & 6 & 0.067 & $2.8750 \mathrm{E}+00$ \\
\hline OBSTCLBM (100) & 3 & 4 & 3 & 3 & 0.043 & $2.8750 \mathrm{E}+00$ & 2 & 2 & 3 & 0.060 & $2.8750 \mathrm{E}+00$ \\
\hline OBSTCLBU (100) & 4 & 6 & 3 & 3 & 0.036 & $2.8750 \mathrm{E}+00$ & 2 & 2 & 1 & 0.053 & $2.8750 \mathrm{E}+00$ \\
\hline TORSION1 (100) & 5 & 6 & 3 & 3 & 0.040 & $-4.9234 \mathrm{E}-01$ & 3 & 3 & 2 & 0.059 & $-4.9234 \mathrm{E}-01$ \\
\hline TORSION2 (100) & 9 & 7 & 4 & 4 & 0.061 & $-4.9234 \mathrm{E}-01$ & 4 & 4 & 2 & 0.073 & $-4.9234 \mathrm{E}-01$ \\
\hline TORSION3 (100) & 3 & 4 & 2 & 2 & 0.022 & $-1.2705 \mathrm{E}+00$ & 2 & 2 & 1 & 0.051 & $-1.2705 \mathrm{E}+00$ \\
\hline TORSION4 (100) & 7 & 5 & 3 & 3 & 0.046 & $-1.2705 \mathrm{E}+00$ & 4 & 4 & 2 & 0.066 & $-1.2705 \mathrm{E}+00$ \\
\hline TORSION5 (100) & 1 & 1 & 0 & 0 & 0.002 & $-2.8971 \mathrm{E}+00$ & 1 & 1 & 0 & 0.044 & $-2.8971 \mathrm{E}+00$ \\
\hline TORSION6 (100) & 2 & 3 & 1 & 1 & 0.025 & $-2.8971 \mathrm{E}+00$ & 3 & 3 & 0 & 0.056 & $-2.8971 \mathrm{E}+00$ \\
\hline TORSIONA (100) & 5 & 6 & 3 & 3 & 0.050 & $-4.0570 \mathrm{E}-01$ & 3 & 3 & 2 & 0.066 & $-4.0570 \mathrm{E}-01$ \\
\hline TORSIONB (100) & 5 & 5 & 5 & 5 & 0.095 & $-4.0570 \mathrm{E}-01$ & 5 & 5 & 6 & 0.114 & $-4.0570 \mathrm{E}-01$ \\
\hline TORSIONC (100) & 3 & 4 & 2 & 2 & 0.027 & $-1.1766 \mathrm{E}+00$ & 2 & 2 & 1 & 0.054 & $-1.1766 \mathrm{E}+00$ \\
\hline TORSIOND (100) & 7 & 5 & 3 & 3 & 0.054 & $-1.1766 \mathrm{E}+00$ & 4 & 4 & 3 & 0.076 & $-1.1766 \mathrm{E}+00$ \\
\hline TORSIONE (100) & 1 & 1 & 0 & 0 & 0.002 & $-2.7984 \mathrm{E}+00$ & 1 & 1 & 0 & 0.045 & $-2.7984 \mathrm{E}+00$ \\
\hline TORSIONF (100) & 2 & 3 & 1 & 1 & 0.029 & $-2.7984 \mathrm{E}+00$ & 3 & 3 & 0 & 0.059 & $-2.7984 \mathrm{E}+00$ \\
\hline HS110 (100) & 2 & 3 & 1 & 2 & 0.109 & $-9.9800 \mathrm{E}+19$ & 2 & 2 & 0 & 0.054 & $-9.9800 \mathrm{E}+19$ \\
\hline NONSCOMP (100) & 47 & 25 & 7 & 7 & 0.337 & $8.0101 \mathrm{E}-12$ & 9 & 9 & 8 & 0.130 & $2.6015 \mathrm{E}-16$ \\
\hline BDEXP (100) & 13 & 12 & 12 & 16 & 0.696 & $1.3497 \mathrm{E}-05$ & 11 & 11 & 10 & 0.192 & $3.9646 \mathrm{E}-05$ \\
\hline EXPLIN (120) & 29 & 47 & 25 & 31 & 0.107 & $-7.2376 \mathrm{E}+05$ & 14 & 14 & 61 & 0.113 & $-7.2324 \mathrm{E}+05$ \\
\hline EXPLIN2 (120) & 17 & 19 & 13 & 61 & 0.056 & $-7.2446 \mathrm{E}+05$ & 12 & 12 & 30 & 0.093 & $-7.2446 \mathrm{E}+05$ \\
\hline EXPQUAD (120) & 22 & 24 & 17 & 32 & 1.642 & $-3.6260 \mathrm{E}+06$ & 18 & 15 & 49 & 0.354 & $-3.6260 \mathrm{E}+06$ \\
\hline HADAMALS (100) & 29 & 43 & 26 & 49 & 2.904 & $2.5316 \mathrm{E}+01$ & 14 & 14 & 370 & 2.610 & $2.5316 \mathrm{E}+01$ \\
\hline MCCORMCK (100) & 8 & 7 & 7 & 12 & 0.472 & $-9.1788 \mathrm{E}+01$ & 7 & 6 & 5 & 0.117 & $-9.1788 \mathrm{E}+01$ \\
\hline PROBPENL (100) & 4 & 5 & 4 & 13 & 0.673 & $-4.9571 \mathrm{E}-06$ & 91 & 52 & 268 & 4.923 & $-2.8726 \mathrm{E}+05$ \\
\hline QRTQUAD (120) & 56 & 70 & 42 & 66 & 3.783 & $-3.6246 \mathrm{E}+06$ & 205 & 168 & 195 & 2.469 & $-3.6242 \mathrm{E}+06$ \\
\hline S368 (100) & 7 & 10 & 7 & 10 & 6.630 & $-1.4869 \mathrm{E}+02$ & 9 & 7 & 9 & 7.007 & $-1.3369 \mathrm{E}+02$ \\
\hline SINEALI (100) & 11 & 12 & 10 & 24 & 0.890 & $-9.9010 \mathrm{E}+03$ & 13 & 9 & 6 & 0.138 & $-9.9010 \mathrm{E}+03$ \\
\hline
\end{tabular}

Tabela 5.3: Desempenho de BETRA e LANCELOT nos problemas com mais de 100 variáveis. 


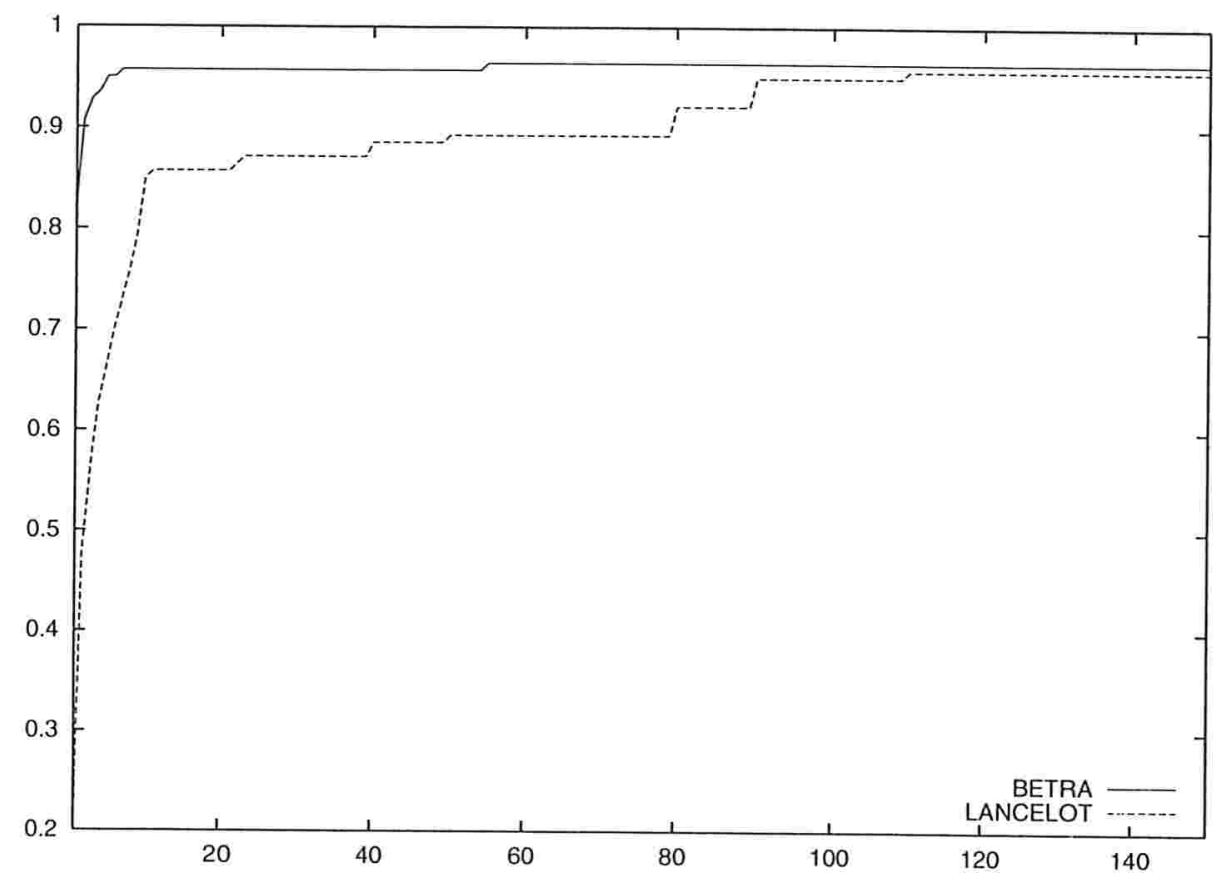

Figura 5.1: Curva do perfil de desempenho comparando BETRA e LANCELOT para todos os problemas

é o mais rápido dentre todos os comparados) e à esquerda medimos a robustez (verificamos a porcentagem de problemas que cada método foi capaz de resolver).

Para a construção do gráfico 5.1, levou-se em conta não somente o tempo de CPU gasto por cada método, mas também a solução encontrada por cada um deles. Quando tanto BETRA como LANCELOT encontram o mesmo valor de função, comparamos o tempo gasto por cada um para encontrá-la. Quando encontram soluções diferentes, considera-se que aquele que encontrou solução pior (maior valor de função) levou tempo "infinito" para chegar à solução. Definimos que dois valores de função $f_{1}$ e $f_{2}$ são iguais se e somente se

$$
\left|f_{1}-f_{2}\right| \leq \max \left\{10^{-10}, 10^{-6} \min \left\{\left|f_{1}\right|,\left|f_{2}\right|\right\}\right\} \text {. }
$$

No gráfico 5.2, utilizando a mesma definição de igualdade de soluções apresentada acima, comparamos os tempos gastos pelos dois métodos apenas nos problemas nos quais ambos obtiveram a mesma solução e ambos pararam sua execução com sucesso (perfazendo um total de 127 problemas)

No gráfico 5.1 podemos observar que BETRA é mais rápido que LANCELOT em $82.14 \%$ dos problemas, enquanto LANCELOT foi mais rápido que BETRA em 19.28\% dos problemas. Pode-se verificar também que BETRA solucionou $96.43 \%$ dos problemas enquanto LANCELOT solucionou $95.71 \%$. Considerando apenas os problemas presentes no gráfico 5.2 , BETRA é mais rápido que LANCELOT em $85.04 \%$ dos casos e LANCELOT é mais rápido que BETRA em $16.53 \%$. 


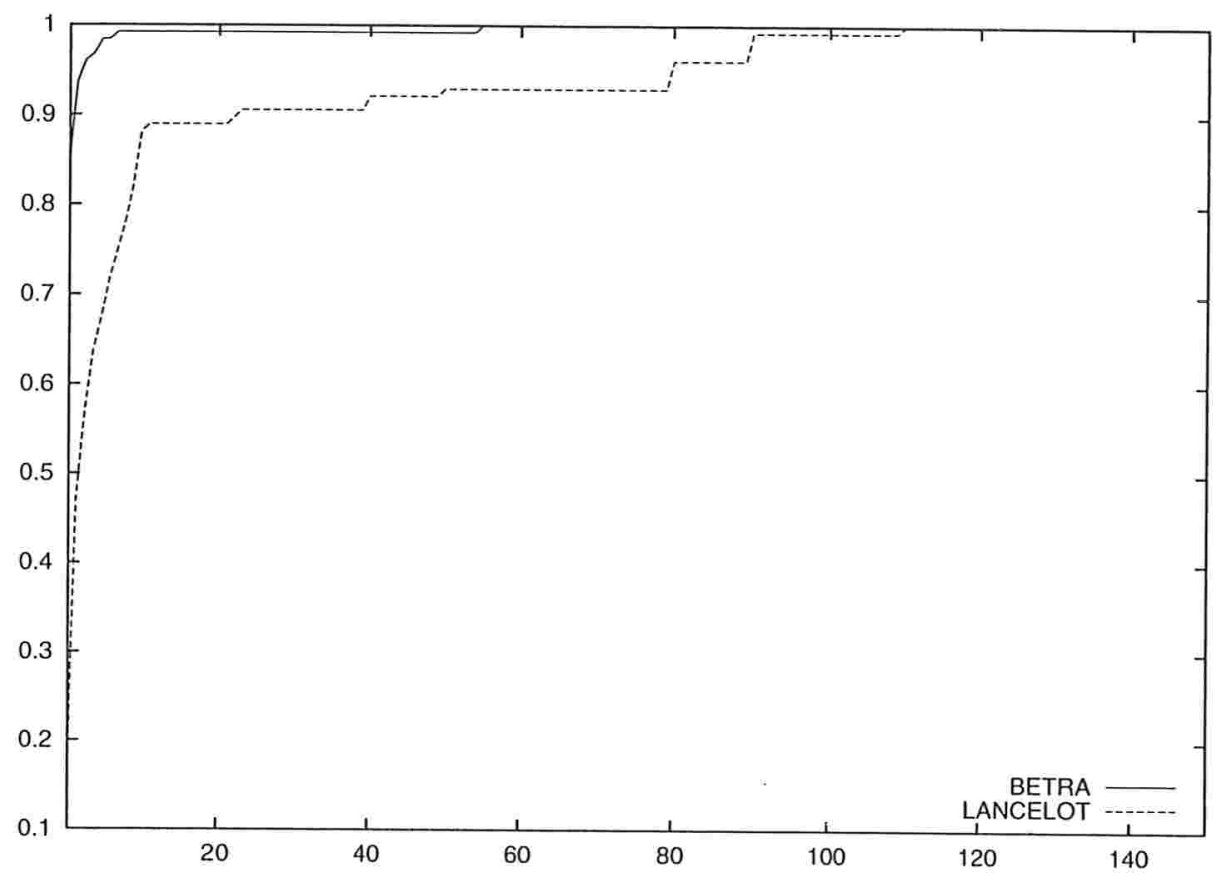

Figura 5.2: Curva do perfil de desempenho comparando BETRA e LANCELOT somente para os problemas nos quais ambos pararam com sucesso e com a mesma solução

É importante notar que, quando se aumenta o valor de $\eta$, o número de iterações do SPG global tende a aumentar, e, quando se aumenta o valor de $\Delta_{m i n}$, o número de iterações do SPG interno também tende a aumentar. Apesar disso, quando aumentamos o valor desses 2 parâmetros, apenas para um pequeno número de problemas o BETRA deixa de encontrar como solução um ponto que satisfaz condições necessárias de segunda ordem. Como exemplo, testamos os 140 problemas mantendo os parâmetros escolhidos para os outros experimentos e modificando apenas os valores de $\eta$ e $\Delta_{\min }$. Para $\eta=0.01$ e $\Delta_{\min }=10^{-5}, 33$ problemas pararam pelo critério 1,5 pelo critério 2, 97 pelo critério 3 , nenhum pelo critério 4 e 5 atingiram o número máximo de iterações. Para $\eta=0.99$ e $\Delta_{\min }=10^{-3}$, o número de problemas que pararam satisfazendo os critérios 1 e 2 se manteve, o número de problemas que pararam pelo critério 3 passou para 96, o número de problemas que pararam pelo critério 4 passou para 2 e o número de problemas que atingiram o número máximo de iterações passou para 4. Portanto, apesar de não ser fácil a escolha destes dois parâmetros, a robustez do BETRA não depende fortemente deles. 


\section{Conclusões e trabalho futuro}

Apresentamos um método prático de restrições ativas para resolver problemas de minimização suave com restrições de caixa, onde o algoritmo interno é um método de regiões de confiança euclidiano clássico e as iterações para deixar as faces são do tipo Gradiente Espectral Projetado. Passos de Gradiente Espectral Projetado também são usados quando algumas restrições inativas são "quase" ativas. Provamos convergência a pontos estacionários de primeira ordem e, sob hipóteses adequadas, a pontos estacionários de segunda ordem. No último caso, os resultados de ordem de convergência de métodos de regiões de confiança de segunda ordem são mantidos.

Comparamos o método com um algoritmo conhecido que usa hessianas verdadeiras e precondicionador completo (ou seja, decomposições de Cholesky) para resolver problemas com restrições de caixa. Os resultados são promissores.

Regiões de confiança euclidianas e elipsoidais têm a vantagem, sobre regiões de confiança com caixas, de minimizadores globais dos subproblemas correspondentes poderem ser obtidos utilizando algoritmos estáveis e eficientes. Por outro lado, bolas e elipsóides não apresentam a mesma forma do domínio do problema, como acontece com regiões de confiança com caixas. Portanto, a vantagem de cada uma das abordagens na prática é controversa e, provavelmente, a decisão correta depende fortemente do problema.

Neste trabalho optamos por regiões de confiança euclidianas, mas também observamos que não há razões para manter os iterandos interiores, com tanto que tenhamos procedimentos eficientes para deixar as faces e para extrapolação, ou seja, para ganhar e abandonar restrições. Aparentemente os resultados numéricos confirmam que esta abordagem é válida para minimização geral com restrições em caixa.

A continuação deste trabalho segue em duas direções: primeiro, considerar problemas de grande porte usando "solvers" de regiões de confiança de grande porte [20]. Segundo, estender a estratégia apresentada neste trabalho para politopos em geral. 


\section{Apêndice A}

\section{Provas dos lemas do capítulo 1}

Lema 1.1.1 Se p é uma solução para (1.1) então p é uma solução para uma equação da forma

$$
(B+\lambda I) p=-g
$$

com $B+\lambda I$ semidefinida positiva, $\lambda \geq 0$ e $\lambda(\Delta-\|p\|)=0$.

Prova Seja $p$ uma solução de (1.1).

Como o problema (1.1) é um problema de minimização com restrições, temos que $p$ é solução de (1.1) se e somente se $p$ satisfaz as condições necessárias de primeira ordem, ou seja, as condições de Karush-Kuhn-Tucker, a saber,

$$
\begin{aligned}
\nabla_{w} \mathcal{L}(p, \lambda) & =0 \\
c(p) & \geq 0 \\
\lambda & \geq 0 \\
c(p) \lambda & =0
\end{aligned}
$$

onde $c(w)=\Delta-\|w\|$ e $\mathcal{L}(w, \lambda)=\varphi(w)-\lambda c(w)$.

Note que

$$
\nabla_{w} \mathcal{L}(w, \lambda)=\nabla_{w} \varphi(w)-\lambda \nabla_{w} c(w)
$$

Como $\nabla_{w} \varphi(w)=B w+g$ e $\nabla_{w} c(w)=-2 w$, temos que

$$
\nabla_{w} \mathcal{L}(w, \lambda)=B w+g+2 \lambda w .
$$

Assim, por (A.1), temos

$$
\nabla_{w} \mathcal{L}(p, \lambda)=B p+g+2 \lambda p=0 .
$$


Ou seja,

$$
B p+2 \lambda p=-g \Rightarrow(B+2 \lambda I) p=-g .
$$

Por (A.4) temos que $c(p) \lambda=0$. Ou seja,

$$
(\Delta-\|p\|) \lambda=0 \Rightarrow 2 \lambda(\Delta-\|p\|)=0 .
$$

Por (A.3) temos que $\lambda \geq 0$. Assim, temos que $2 \lambda \geq 0$.

Trocando $2 \lambda$ por $\lambda$ temos que $p$ satisfaz (1.2) $\operatorname{com} \lambda \geq 0$ e $\lambda(\Delta-\|p\|)=0$.

Agora basta provar que $B+\lambda I$ é semidefinida positiva.

Suponha que $p \neq 0$. Como $p$ é solução de (1.1), temos que $p$ é solução de

$$
\begin{array}{ll}
\operatorname{minimizar} & \varphi(w) \\
\text { sujeita a } & \|w\| \leq\|p\| .
\end{array}
$$

Ou seja, para qualquer $w$ com $\|w\|=\|p\|$, temos que

$$
\begin{gathered}
\varphi(w) \geq \varphi(p) \Rightarrow \\
\frac{1}{2} w^{T} B w+g^{T} w \geq \frac{1}{2} p^{T} B p+g^{T} p .
\end{gathered}
$$

Como $g=-(B+\lambda I) p$, temos a seguinte desigualdade

$$
\begin{aligned}
\frac{1}{2} w^{T} B w-((B+\lambda I) p)^{T} w & \geq \frac{1}{2} p^{T} B p-((B+\lambda I) p)^{T} p \Rightarrow \\
\frac{1}{2} w^{T} B w-p^{T}(B+\lambda I)^{T} w & \geq \frac{1}{2} p^{T} B p-p^{T}(B+\lambda I)^{T} p .
\end{aligned}
$$
temos

Como $B$ é simétrica, $B+\lambda I$ também é simétrica, ou seja, $(B+\lambda I)^{T}=(B+\lambda I)$. Assim,

$$
\begin{gathered}
\frac{1}{2} w^{T} B w-p^{T}(B+\lambda I) w \geq \frac{1}{2} p^{T} B p-p^{T}(B+\lambda I) p \Rightarrow \\
\frac{1}{2} w^{T} B w-p^{T} B w-\lambda p^{T} w \geq \frac{1}{2} p^{T} B p-p^{T} B p-\lambda p^{T} p=-\frac{1}{2} p^{T} B p-\lambda p^{T} p \Rightarrow
\end{gathered}
$$




$$
\begin{gathered}
\frac{1}{2} w^{T} B w-p^{T} B w+\frac{1}{2} p^{T} B p \geq-\lambda p^{T} p+\lambda p^{T} w \Rightarrow \\
\frac{1}{2}(w-p)^{T} B(w-p) \geq-\lambda p^{T} p+\lambda p^{T} w .
\end{gathered}
$$

$\operatorname{Mas} \frac{1}{2}(w-p)^{T} B(w-p)=\frac{1}{2}(w-p)^{T}(B+\lambda I)(w-p)-\frac{\lambda}{2}(w-p)^{T}(w-p)$.

$$
\begin{gathered}
\frac{1}{2}(w-p)^{T}(B+\lambda I)(w-p)-\frac{\lambda}{2}(w-p)^{T}(w-p) \geq-\lambda p^{T} p+\lambda p^{T} w \Rightarrow \\
\frac{1}{2}(w-p)^{T}(B+\lambda I)(w-p) \geq-\lambda p^{T} p+\lambda p^{T} w+\frac{\lambda}{2}(w-p)^{T}(w-p) \Rightarrow \\
\frac{1}{2}(w-p)^{T}(B+\lambda I)(w-p) \geq-\lambda p^{T} p+\lambda p^{T} w+\frac{\lambda}{2} w^{T} w-\lambda p^{T} w+\frac{\lambda}{2} p^{T} p \Rightarrow \\
\frac{1}{2}(w-p)^{T}(B+\lambda I)(w-p) \geq \frac{\lambda}{2}\left(w^{T} w-p^{T} p\right)=\frac{\lambda}{2}\left(\|w\|^{2}-\|p\|^{2}\right) .
\end{gathered}
$$

Como $\|w\|=\|p\|$, temos que

$$
\frac{1}{2}(w-p)^{T}(B+\lambda I)(w-p) \geq 0 .
$$

Assim, $B+\lambda I$ é semidefinida positiva quando $p \neq 0$.

Se $p=0$, como $g=-(B+\lambda I) p$, temos que $g=0$.

Desta forma, como $p$ é solução de (1.1), temos que $p$ também é solução de

$$
\begin{array}{ll}
\text { minimizar } & \frac{1}{2} w^{T} B w \\
\text { sujeita a } & \|w\| \leq \Delta
\end{array}
$$

Mas (A.6) só tem solução se $B$ é semidefinida positiva. Como $\lambda \geq 0, B+\lambda I$ também é semidefinida positiva.

Lema 1.1.2 Sejam $\lambda \in \mathbb{R}$ e $p \in \mathbb{R}^{n}$ satisfazendo (1.2) com $B+\lambda I$ semidefinida positiva.

(i) Se $\lambda=0$ e $\|p\| \leq \Delta$ então p é solução de (1.1);

(ii) $p$ é solução de $\min \{\varphi(w):\|w\| \leq\|p\|\}$;

(iii) se $\lambda \geq 0$ e $\|p\|=\Delta$ então $p$ é solução de (1.1). 
Se $B+\lambda I$ é definida positiva, $p$ é único em (i), (ii) e (iii).

Prova Se $(B+\lambda I) p=-g$ e $B+\lambda I$ é semidefinida positiva, $p$ é o mínimo de uma quadrática que tem como hessiana $B+\lambda I$ e $(B+\lambda I) w+g$ como gradiente.

Ou seja, $p$ é o minimizador da quadrática

$$
\frac{1}{2} w^{T}(B+\lambda I) w+g^{T} w
$$

Neste caso, para qualquer $w \in \mathbb{R}^{n}$ vale que

$$
\begin{gathered}
\frac{1}{2} w^{T}(B+\lambda I) w+g^{T} w \geq \frac{1}{2} p^{T}(B+\lambda I) p+g^{T} p \Rightarrow \\
\frac{1}{2} w^{T} B w+\frac{\lambda}{2} w^{T} w+g^{T} w \geq \frac{1}{2} p^{T} B p+\frac{\lambda}{2} p^{T} p+g^{T} p .
\end{gathered}
$$

Como

$$
\frac{1}{2} w^{T} B w+g^{T} w=\varphi(w) \quad \text { e } \quad \frac{1}{2} p^{T} B p+g^{T} p=\varphi(p),
$$

temos que

$$
\begin{gathered}
\varphi(w)+\frac{\lambda}{2} w^{T} w \geq \varphi(p)+\frac{\lambda}{2} p^{T} p \Rightarrow \\
\varphi(w) \geq \varphi(p)+\frac{\lambda}{2} p^{T} p-\frac{\lambda}{2} w^{T} w \Rightarrow \\
\varphi(w) \geq \varphi(p)+\frac{\lambda}{2}\left(p^{T} p-w^{T} w\right) .
\end{gathered}
$$

Agora, com base na inequação (A.7), podem-se provar os itens (i), (ii) e (iii) do Lema 1.1.2.

(i) Se $\lambda=0$ então, por (A.7), temos que $\varphi(w) \geq \varphi(p)$, ou seja, $p$ é minimizador irrestrito de $\varphi(w)$. Como $\|p\| \leq \Delta$, temos que $p$ é solução de (1.1).

(ii) Se considerarmos apenas os vetores $w \in \mathbb{R}^{n}$ tais que $\|w\| \leq\|p\|$, temos que $\|p\|^{2}-\|w\|^{2} \geq$ 0. Por (A.7),

$$
\varphi(w) \geq \varphi(p)+\frac{\lambda}{2}\left(p^{T} p-w^{T} w\right) \geq \varphi(p)
$$


Portanto, $p$ é solução de $\min \{\varphi(w):\|w\| \leq\|p\|\}$;

(iii) se $\|p\|=\Delta$ então, pelo item (ii), temos que $p$ é solução de (1.1).

É fácil ver que se $B+\lambda I$ é definida positiva, então as soluções para (i), (ii) e (iii) são únicas, pois na inequação (A.7) tem-se " $<$ " no lugar de " $\leq$ ".

Lema 1.1.3 O problema (1.1) não tem solução na borda de $\{w:\|w\| \leq \Delta\}$ se e somente se $B$ é definida positiva e $\left\|B^{-1} g\right\|<\Delta$.

\section{Prova}

(i) Se $B$ é definida positiva e $\left\|B^{-1} g\right\|<\Delta$ então o problema (1.1) não tem solução na borda de $\{w:\|w\| \leq \Delta\}$.

Se $B$ é definida positiva, $\varphi(w)$ é convexa e seu único ponto estacionário é um minimizador, dado por $p=-B^{-1} g$. Se $\|p\|<\Delta$ então $p$ é o minimizador de $\varphi(w)$ sujeita a $\|w\| \leq \Delta$. Ou seja, $p$ é solução do problema (1.1) que não está na borda de $\{w:\|w\| \leq \Delta\}$.

(ii) Se o problema (1.1) não tem solução na borda de $\{w:\|w\| \leq \Delta\}$ então $B$ é definida positiva e $\left\|B^{-1} g\right\|<\Delta$.

Seja $p$ uma solução do problema (1.1).

Como, por hipótese, (1.1) não tem solução na borda de $\{w:\|w\| \leq \Delta\}$, temos que $\|p\|<\Delta$.

Como vale o Lema 1.1.1, temos que

$$
\lambda(\Delta-\|p\|)=0 \Rightarrow \lambda=0 .
$$

Além disso, temos que $B+\lambda I$ é semidefinida positiva. Como $\lambda=0$, na verdade $B$ é semidefinida positiva.

Agora basta provar que $B$ é não-singular. Desta forma temos que $B$ é definida positiva e, portanto, inversível. Daí, temos que, como $B p=-g, p=-B^{-1} g$ que, por hipótese, tem norma menor do que $\Delta$.

Suponha, por absurdo, que $B$ seja singular. Neste caso, existe algum $z$ com $\|p+z\|=\Delta$ tal que $B z=0$.

Mas como $\lambda=0$ e $B+\lambda I$ é semidefinida positiva, pelo Lema 1.1.2-(iii) temos que $(p+z)$ é solução de (1.1) na borda, o que contradiz a hipótese de que (1.1) não tem solução na borda. 
Portanto, $B$ é não-singular. Ou seja, $B$ é definida positiva.

Lema 1.1.4 Seja $p(\lambda)=-(B+\lambda I)^{-1} g$ com $B+\lambda I$ definida positiva.

Tome a decomposição $B=Q \Lambda Q^{T}, \operatorname{com} \Lambda=\operatorname{diag}\left(\lambda_{1}, \lambda_{2}, \ldots, \lambda_{n}\right)$ e $Q^{T} Q=I$, onde $\lambda_{1} \leq$ $\lambda_{2} \leq \ldots \leq \lambda_{n}$ são os autovalores de $B$ (que existe porque $B$ é simétrica).

Então, para $\lambda \neq-\lambda_{j}$,

$$
p(\lambda)=-\sum_{j=1}^{n} \frac{q_{j}^{T} g}{\lambda_{j}+\lambda} q_{j}
$$

onde $q_{j}$ é a j-ésima coluna de $Q$.

Prova Como $B=Q \Lambda Q^{T}$, temos que

$$
B+\lambda I=Q \Lambda Q^{T}+\lambda I .
$$

Além disso, $Q^{T} Q=I$. Assim,

$$
B+\lambda I=Q \Lambda Q^{T}+\lambda Q^{T} Q=Q \Lambda Q^{T}+\lambda Q Q^{T}=Q \Lambda Q^{T}+Q(\lambda I) Q^{T}=Q(\Lambda+\lambda I) Q^{T} .
$$

Então temos que

$$
p(\lambda)=-\left(Q(\Lambda+\lambda I) Q^{T}\right)^{-1} g=-Q^{-T}(\Lambda+\lambda I)^{-1} Q^{-1} g=-Q(\Lambda+\lambda I)^{-1} Q^{T} g .
$$

Note que

$$
(\Lambda+\lambda I)^{-1}=\left[\begin{array}{cccc}
\frac{1}{\lambda_{1}+\lambda} & 0 & \cdots & 0 \\
0 & \frac{1}{\lambda_{2}+\lambda} & \cdots & 0 \\
\vdots & \vdots & \ddots & \vdots \\
0 & 0 & \cdots & \frac{1}{\lambda_{n}+\lambda}
\end{array}\right]
$$

Assim,

$$
\begin{aligned}
& p(\lambda)=-Q\left[\begin{array}{cccc}
\frac{1}{\lambda_{1}+\lambda} & 0 & \cdots & 0 \\
0 & \frac{1}{\lambda_{2}+\lambda} & \cdots & 0 \\
\vdots & \vdots & \ddots & \vdots \\
0 & 0 & \cdots & \frac{1}{\lambda_{n}+\lambda}
\end{array}\right] Q^{T} g= \\
& p(\lambda)=-\left[\frac{1}{\lambda_{1}+\lambda} q_{1}\left|\frac{1}{\lambda_{2}+\lambda} q_{2}\right| \cdots \mid \frac{1}{\lambda_{n}+\lambda} q_{n}\right] Q^{T} g \text {, }
\end{aligned}
$$


onde $q_{j}$ é a $j$-ésima coluna de $Q$.

$$
p(\lambda)=-\left[\frac{1}{\lambda_{1}+\lambda} q_{1}\left|\frac{1}{\lambda_{2}+\lambda} q_{2}\right| \cdots \mid \frac{1}{\lambda_{n}+\lambda} q_{n}\right]\left[\begin{array}{c}
q_{1}^{T} g \\
q_{2}^{T} g \\
\vdots \\
q_{n}^{T} g
\end{array}\right]=-\sum_{j=1}^{n} \frac{q_{j}^{T} g}{\lambda_{j}+\lambda} q_{j},
$$

$\operatorname{para} \lambda \neq-\lambda_{j}$.

Lema 1.2.1 Seja $\phi(\lambda)=\frac{1}{\Delta}-\frac{1}{\|p(\lambda)\|}$, onde $p(\lambda)=-(B+\lambda I)^{-1} g$ com $B$ simétrica e $B+\lambda I$ definida positiva.

Então,

$$
-\frac{\phi(\lambda)}{\phi^{\prime}(\lambda)}=\left(\frac{\|p(\lambda)\|}{\|t(\lambda)\|}\right)^{2}\left(\frac{\|p(\lambda)\|-\Delta}{\Delta}\right)
$$

onde $t(\lambda)$ é a solução de $R^{T} t(\lambda)=p(\lambda)$ e $R$ é a matriz triangular superior tal que $B+\lambda I=$ $R^{T} R$.

Prova Primeiramente note que, se $B+\lambda I$ é definida positiva, necessariamente temos que $\lambda \neq-\lambda_{j}$, para $j=1, \ldots, n$.

Assim, pelo Lema 1.1.4, temos que $p(\lambda)=-\sum_{j=1}^{n} \frac{q_{j}^{T} g}{\lambda_{j}+\lambda} q_{j}$.

Note que

$$
\|p(\lambda)\|^{2}=\left(\sum_{j=1}^{n} \frac{q_{j}^{T} g}{\lambda_{j}+\lambda} q_{j}\right)^{T}\left(\sum_{j=1}^{n} \frac{q_{j}^{T} g}{\lambda_{j}+\lambda} q_{j}\right) .
$$

Como $Q^{T} Q=I$, temos que $\left(q_{i}\right)^{T} q_{i}=1$ para todo $i$ e $\left(q_{j}\right)^{T} q_{i}=0$ para todo $j \neq i$. Assim,

$$
\|p(\lambda)\|^{2}=\sum_{j=1}^{n} \frac{\left(q_{j}^{T} g\right)^{2}}{\left(\lambda_{j}+\lambda\right)^{2}}
$$

É fácil verificar que

$$
\left(\|p(\lambda)\|^{2}\right)^{\prime}=-2 \sum_{j=1}^{n} \frac{\left(q_{j}^{T} g\right)^{2}}{\left(\lambda_{j}+\lambda\right)^{3}} .
$$

Vejamos agora como podemos escrever $\phi^{\prime}(\lambda)$.

$$
\phi^{\prime}(\lambda)=-\left(\frac{1}{\|p(\lambda)\|}\right)^{\prime}=-\left(\left(\|p(\lambda)\|^{2}\right)^{-\frac{1}{2}}\right)^{\prime}=\frac{1}{2}\left(\|p(\lambda)\|^{2}\right)^{-\frac{3}{2}}\left(\|p(\lambda)\|^{2}\right)^{\prime}=\frac{1}{2}\|p(\lambda)\|^{-3}\left(\|p(\lambda)\|^{2}\right)^{\prime} .
$$


Usando a equação (A.8), obtemos

$$
\phi^{\prime}(\lambda)=\frac{1}{2}\|p(\lambda)\|^{-3}\left(-2 \sum_{j=1}^{n} \frac{\left(q_{j}^{T} g\right)^{2}}{\left(\lambda_{j}+\lambda\right)^{3}}\right)=-\|p(\lambda)\|^{-3}\left(\sum_{j=1}^{n} \frac{\left(q_{j}^{T} g\right)^{2}}{\left(\lambda_{j}+\lambda\right)^{3}}\right) .
$$

Observe ainda que

$$
\begin{gathered}
\|t(\lambda)\|^{2}=\left\|R^{-T} p(\lambda)\right\|^{2}=\left(R^{-T} p(\lambda)\right)^{T} R^{-T} p(\lambda)=p(\lambda)^{T} R^{-1} R^{-T} p(\lambda)= \\
p(\lambda)^{T}\left(R^{T} R\right)^{-1} p(\lambda)=p(\lambda)^{T}(B+\lambda I)^{-1} p(\lambda) .
\end{gathered}
$$

Como $p(\lambda)=-\sum_{j=1}^{n} \frac{q_{j}^{T} g}{\lambda_{j}+\lambda} q_{j}$ (pelo Lema 1.1.4), temos que

$$
\|t(\lambda)\|^{2}=\left(-\sum_{j=1}^{n} \frac{q_{j}^{T} g}{\lambda_{j}+\lambda} q_{j}\right)^{T}(B+\lambda I)^{-1}\left(-\sum_{j=1}^{n} \frac{q_{j}^{T} g}{\lambda_{j}+\lambda} q_{j}\right) .
$$

Lembrando que $(B+\lambda I)^{-1}=Q(\Lambda+\lambda I)^{-1} Q^{T}$ (veja prova do Lema 1.1.4), temos que

$$
\|t(\lambda)\|^{2}=\left(-\sum_{j=1}^{n} \frac{q_{j}^{T} g}{\lambda_{j}+\lambda} q_{j}\right)^{T} Q(\Lambda+\lambda I)^{-1} Q^{T}\left(-\sum_{j=1}^{n} \frac{q_{j}^{T} g}{\lambda_{j}+\lambda} q_{j}\right) .
$$

Como $Q$ é ortonormal, temos que

$$
\begin{gathered}
\|t(\lambda)\|^{2}=\left(-\sum_{j=1}^{n} \frac{q_{j}^{T} g}{\lambda_{j}+\lambda} e_{j}^{T}\right)(\Lambda+\lambda I)^{-1}\left(-\sum_{j=1}^{n} \frac{q_{j}^{T} g}{\lambda_{j}+\lambda} e_{j}\right)= \\
\left(-\sum_{j=1}^{n} \frac{q_{j}^{T} g}{\left(\lambda_{j}+\lambda\right)^{2}} e_{j}^{T}\right)\left(-\sum_{j=1}^{n} \frac{q_{j}^{T} g}{\lambda_{j}+\lambda} e_{j}\right)=\sum_{j=1}^{n} \frac{\left(q_{j}^{T} g\right)^{2}}{\left(\lambda_{j}+\lambda\right)^{3}} .
\end{gathered}
$$

Substituindo em (A.9), temos que

$$
\phi^{\prime}(\lambda)=-\|p(\lambda)\|^{-3}\|t(\lambda)\|^{2}=-\frac{\|t(\lambda)\|^{2}}{\|p(\lambda)\|^{3}} .
$$

Finalmente, de posse destes resultados, analisemos $-\frac{\phi(\lambda)}{\phi^{\prime}(\lambda)}$. 


$$
\begin{gathered}
-\frac{\phi(\lambda)}{\phi^{\prime}(\lambda)}=-\frac{\left(\frac{1}{\Delta}-\frac{1}{\|p(\lambda)\|}\right)}{-\left(\frac{\|t(\lambda)\|^{2}}{\|p(\lambda)\|^{3}}\right)}=\left(\frac{1}{\Delta}-\frac{1}{\|p(\lambda)\|}\right)\left(\frac{\|p(\lambda)\|^{3}}{\|t(\lambda)\|^{2}}\right)= \\
\left(\frac{\|p(\lambda)\|-\Delta}{\Delta\|p(\lambda)\|}\right)\left(\frac{\|p(\lambda)\|^{3}}{\|t(\lambda)\|^{2}}\right)=\left(\frac{\|p(\lambda)\|}{\|t(\lambda)\|}\right)^{2}\left(\frac{\|p(\lambda)\|-\Delta}{\Delta}\right) .
\end{gathered}
$$

Lema 1.2.2 Seja $0<\sigma<1$ dado e suponha que

$$
B+\lambda I=R^{T} R, \quad(B+\lambda I) p=-g, \quad \lambda \geq 0 .
$$

Seja $z \in \mathbb{R}^{n}$ tal que

$$
\|p+z\|=\Delta, \quad\|R z\|^{2} \leq \sigma\left(\|R p\|^{2}+\lambda \Delta^{2}\right) .
$$

Então

$$
-\varphi(p+z) \geq \frac{1}{2}(1-\sigma)\left(\|R p\|^{2}+\lambda \Delta^{2}\right) \geq(1-\sigma)\left|\varphi^{*}\right|,
$$

onde $\varphi^{*}$ é o valor ótimo de (1.1).

Prova Primeiramente calculemos $\varphi(p+z)$ :

$$
\begin{gathered}
\varphi(p+z)=\frac{1}{2}(p+z)^{T} B(p+z)+g^{T}(p+z)= \\
\frac{1}{2} p^{T} B p+z^{T} B p+\frac{1}{2} z^{T} B z+g^{T} p+g^{T} z .
\end{gathered}
$$

Como, por hipótese, $g=-(B+\lambda I) p=-R^{T} R p$, temos

$$
\varphi(p+z)=\frac{1}{2} p^{T} B p+z^{T} B p+\frac{1}{2} z^{T} B z+\left(-R^{T} R p\right)^{T} p+\left(-R^{T} R p\right)^{T} z .
$$

Além disso, para quaisquer $x, y \in \mathbb{R}^{n}$ vale que $x^{T} B y=x^{T}(B+\lambda I) y-\lambda x^{T} y=x^{T}\left(R^{T} R\right) y-$ $\lambda x^{T} y$. Então,

$$
\begin{gathered}
\varphi(p+z)=\frac{1}{2} p^{T}\left(R^{T} R\right) p-\frac{\lambda}{2} p^{T} p+z^{T}\left(R^{T} R\right) p-\lambda z^{T} p+\frac{1}{2} z^{T}\left(R^{T} R\right) z-\frac{\lambda}{2} z^{T} z-p^{T} R^{T} R p-p^{T} R^{T} R z= \\
\frac{1}{2}(R p)^{T} R p-\frac{\lambda}{2} p^{T} p+(R z)^{T} R p-\lambda z^{T} p+\frac{1}{2}(R z)^{T} R z-\frac{\lambda}{2} z^{T} z-(R p)^{T} R p-(R z)^{T} R p=
\end{gathered}
$$




$$
\frac{1}{2}\|R p\|^{2}-\frac{\lambda}{2}\|p\|^{2}+(R z)^{T} R p-\lambda z^{T} p+\frac{1}{2}\|R z\|^{2}-\frac{\lambda}{2}\|z\|^{2}-\|R p\|^{2}-(R z)^{T} R p .
$$

Como $\|p+z\|^{2}=\|p\|^{2}+2 p^{T} z+\|z\|^{2}$, concluímos que

$$
\varphi(p+z)=-\frac{1}{2}\left(\|R p\|^{2}+\lambda\|p+z\|^{2}\right)+\frac{1}{2}\|R z\|^{2} .
$$

Para qualquer $z$ que satisfaça (1.6), temos

$$
\begin{gathered}
-\varphi(p+z)=\frac{1}{2}\left(\|R p\|^{2}+\lambda\|p+z\|^{2}\right)-\frac{1}{2}\|R z\|^{2} \geq \\
\frac{1}{2}\left(\|R p\|^{2}+\lambda\|p+z\|^{2}\right)-\frac{1}{2} \sigma\left(\|R p\|^{2}+\lambda \Delta^{2}\right) .
\end{gathered}
$$

Como $z$ satisfaz (1.6), temos que $\|p+z\|=\Delta$. Assim,

$$
-\varphi(p+z) \geq \frac{1}{2}\left(\|R p\|^{2}+\lambda \Delta^{2}\right)-\frac{1}{2} \sigma\left(\|R p\|^{2}+\lambda \Delta^{2}\right)=\frac{1}{2}(1-\sigma)\left(\|R p\|^{2}+\lambda \Delta^{2}\right) .
$$

Ainda temos que, se $\varphi^{*}=\varphi\left(p+z^{*}\right)$, onde $\left\|p+z^{*}\right\| \leq \Delta$,

$$
\begin{gathered}
-\varphi\left(p+z^{*}\right)=\frac{1}{2}\left(\|R p\|^{2}+\lambda\left\|p+z^{*}\right\|^{2}\right)-\frac{1}{2}\left\|R z^{*}\right\|^{2} \leq \\
\frac{1}{2}\left(\|R p\|^{2}+\lambda\left\|p+z^{*}\right\|^{2}\right) \leq \frac{1}{2}\left(\|R p\|^{2}+\lambda \Delta^{2}\right) .
\end{gathered}
$$

Note que $p=0$ sempre é solução de (1.1). Então, certamente $\varphi\left(p+z^{*}\right) \leq 0$, ou seja, $\varphi^{*}=\left|\varphi^{*}\right|$. Assim, temos as duas desigualdades que gostaríamos de provar.

Lema 1.2.3 Seja $(B+\lambda I)=R^{T} R$ uma matriz definida positiva. Sejam $v \in \mathbb{R}^{n}$ o vetor calculado utilizando a técnica descrita em [7] e $\hat{z}=\frac{v}{\|v\|}$. Então $\|R \hat{z}\|$ se aproxima de 0 quando $\lambda$ se aproxima de $-\lambda_{1}$, com $\lambda_{1}$ o menor autovalor de $B$.

Prova Como mencionado na seção 1.2.2, a técnica descrita em [7] constrói um vetor e com componentes 1 ou -1 de forma que a solução $w$ do sistema $R^{T} w=e$ seja grande. Então resolve-se o sistema $R v=w$ para $v$ e calculamos 


$$
\hat{z}=\frac{v}{\|v\|}
$$

Lembre-se que $R$ é o fator de Cholesky de $B+\lambda I$ e, portanto, não é singular.

Para quaisquer vetores $v$ e $w$ tais que $R^{T} w=e$ e $R v=w$ temos que

$$
\|w\|^{2}=\left\|R^{-T} e\right\|^{2}=e^{T} R^{-1} R^{-T} e=e^{T} v \leq\|e\|\|v\| .
$$

Como as componentes de $e$ são todas iguais a 1 ou $-1,\|e\|=\sqrt{n}$. Assim,

$$
\|w\|^{2} \leq \sqrt{n}\|v\| .
$$

Note que, como $\hat{z}=\frac{v}{\|v\|}$, temos

$$
\|R \hat{z}\|=\frac{\|R v\|}{\|v\|}=\frac{\|w\|}{\|v\|} \leq \frac{\sqrt{n}}{\|w\|} .
$$

Vejamos agora como se comporta $\|w\|$. Em [7], quando vamos calcular a componente $w_{k}$, já temos calculados $w_{1}, \ldots, w_{k-1}$. Então definimos

$$
p_{i}^{(k-1)}=\sum_{l=1}^{k-1} r_{l i} w_{l}, \quad k \leq i \leq n
$$

onde $r_{i j}$ é o elemento na posição $(i, j)$ de $R$. Temos então duas escolhas para $w_{k}$, que são

$$
w_{k}^{+}=\frac{1-p_{k}^{(k-1)}}{r_{k k}} \text { e } w_{k}^{-}=\frac{-1-p_{k}^{(k-1)}}{r_{k k}} .
$$

Se

$$
\left|\omega_{k}^{+}\right|+\sum_{i=k+1}^{n}\left|p_{i}^{(k-1)}+r_{k i} \omega_{k}^{+}\right| \geq\left|\omega_{k}^{-}\right|+\sum_{i=k+1}^{n}\left|p_{i}^{(k-1)}+r_{k i} \omega_{k}^{-}\right|
$$

então $w_{k}$ é escolhido como $w_{k}^{+}$. Senão, $w_{k}=w_{k}^{-}$.

Veja que, se $p_{k}^{(k-1)} \leq 0$, temos que

$$
\left|w_{k}^{+}\right| \geq\left|w_{k}^{-}\right| \mathrm{e}\left|w_{k}^{+}\right| \geq \frac{1}{\left|r_{k k}\right|}
$$


Se $p_{k}^{(k-1)} \geq 0$, temos que

$$
\left|w_{k}^{-}\right| \geq\left|w_{k}^{+}\right| \mathrm{e}\left|w_{k}^{-}\right| \geq \frac{1}{\left|r_{k k}\right|} .
$$

Portanto,

e daí segue que

$$
\max \left\{\left|w_{k}^{+}\right|,\left|w_{k}^{-}\right|\right\} \geq \frac{1}{\left|r_{k k}\right|}
$$

$$
\begin{gathered}
\frac{1}{\left|r_{k k}\right|} \leq\left|w_{k}\right|+\sum_{i=k+1}^{n}\left|p_{i}^{(k-1)}+r_{k i} w_{k}\right| \leq(1+r)\|w\| \Rightarrow \\
\frac{1}{\|w\|} \leq(1+r)\left|r_{k k}\right|, \quad 1 \leq k \leq n,
\end{gathered}
$$

onde $r$ é tal que

$$
\sum_{j=1}^{n} \sum_{i=1}^{j}\left|r_{i j}\right| \leq r
$$

Voltando à inequação (A.11), temos que

$$
\|R \hat{z}\| \leq \sqrt{n}(1+r) \min \left\{\left|r_{k k}\right|: 1 \leq k \leq n\right\} .
$$

Ora, quando $\lambda$ se aproxima de $-\lambda_{1}$, algum elemento da diagonal de $R$ se aproxima de 0 e a. equação (A.12) nos diz que $\|R \hat{z}\|$ também se aproxima de 0 , como gostaríamos.

Lema 1.2.4 Tome B, g e $\Delta$ do problema (1.1).

Um limitante inferior $\lambda_{L}$ para $\lambda^{*}$ solução de $\phi(\lambda)$ é

$$
\lambda_{L}=\frac{\|g\|}{\Delta}-\|B\|_{1},
$$

e um limitante superior $\lambda_{U}$ é

$$
\lambda_{U}=\frac{\|g\|}{\Delta}+\|B\|_{1} .
$$


Prova Por (1.3) temos que

$$
\|p(\lambda)\|^{2}=\sum_{j=1}^{n} \frac{\left(q_{j}^{T} g\right)^{2}}{\left(\lambda_{j}+\lambda\right)^{2}} .
$$

Como $\lambda_{1} \leq \lambda_{j} \leq \lambda_{n}$, para todo $j$, temos que

$$
\frac{1}{\left(\lambda_{n}+\lambda\right)^{2}} \sum_{j=1}^{n}\left(q_{j}^{T} g\right)^{2} \leq\|p(\lambda)\|^{2} \leq \frac{1}{\left(\lambda_{1}+\lambda\right)^{2}} \sum_{j=1}^{n}\left(q_{j}^{T} g\right)^{2} .
$$

Note agora que

$$
\sum_{j=1}^{n}\left(q_{j}^{T} g\right)^{2}=\sum_{j=1}^{n}\left[Q^{T} g\right]_{j}^{2}=\left\|Q^{T} g\right\|^{2}
$$

Como $Q$ é ortonormal,

$$
\left\|Q^{T} g\right\|^{2}=\|g\|^{2}
$$

Assim, temos que

$$
\frac{\|g\|^{2}}{\left(\lambda_{n}+\lambda\right)^{2}} \leq\|p(\lambda)\|^{2} \leq \frac{\|g\|^{2}}{\left(\lambda_{1}+\lambda\right)^{2}} .
$$

Como $\lambda^{*}>-\lambda_{1}$ temos que $\lambda^{*}+\lambda_{j}>0$ para todo $j$. Daí segue que

$$
\frac{\|g\|}{\lambda_{n}+\lambda^{*}} \leq\left\|p\left(\lambda^{*}\right)\right\| \leq \frac{\|g\|}{\lambda_{1}+\lambda^{*}} .
$$

Para continuar, vejamos algumas propriedades de $\|B\|_{1}$ (veja, por exemplo, [12]).

Por definição,

$$
\|B\|_{1}=\max _{1 \leq j \leq n} \sum_{i=1}^{n}\left|b_{i j}\right|
$$

e

$$
\|B\|_{\infty}=\max _{1 \leq i \leq n} \sum_{j=1}^{n}\left|b_{i j}\right| .
$$


Existe uma propriedade que diz que $\|B\|_{2} \leq \sqrt{\|B\|_{1}\|B\|_{\infty}}$.

Como $B$ é simétrica, temos que

$$
\|B\|_{1}=\|B\|_{\infty} \Rightarrow\|B\|_{2} \leq \sqrt{\|B\|_{1}^{2}} \Rightarrow\|B\|_{2} \leq\|B\|_{1} .
$$

Uma outra propriedade de normas de matrizes é $\|B\|_{2}=\max \left\{\left|\lambda_{1}\right|,\left|\lambda_{n}\right|\right\}$, quando $B$ é simétrica.

Daí temos que

$$
\begin{aligned}
& \|B\|_{2}=\max \left\{\left|\lambda_{1}\right|,\left|\lambda_{n}\right|\right\} \leq\|B\|_{1} \Rightarrow \\
& \left|\lambda_{1}\right| \leq\|B\|_{1} \Rightarrow-\|B\|_{1} \leq \lambda_{1} \leq\|B\|_{1}
\end{aligned}
$$

e

$$
\left|\lambda_{n}\right| \leq\|B\|_{1} \Rightarrow-\|B\|_{1} \leq \lambda_{n} \leq\|B\|_{1} .
$$

Voltando à inequação à esquerda de (A.13), temos que

$$
\frac{\|g\|}{\lambda_{n}+\lambda^{*}} \leq\left\|p\left(\lambda^{*}\right)\right\| \Rightarrow \frac{\|g\|}{\left\|p\left(\lambda^{*}\right)\right\|} \leq \lambda_{n}+\lambda^{*} \Rightarrow \frac{\|g\|}{\left\|p\left(\lambda^{*}\right)\right\|}-\lambda_{n} \leq \lambda^{*} .
$$

Como $\lambda_{n} \leq\|B\|_{1}$ temos

$$
\lambda^{*} \geq \frac{\|g\|}{\left\|p\left(\lambda^{*}\right)\right\|}-\lambda_{n} \geq \frac{\|g\|}{\left\|p\left(\lambda^{*}\right)\right\|}-\|B\|_{1} .
$$

Como $\left\|p\left(\lambda^{*}\right)\right\|=\Delta$, temos

$$
\lambda^{*} \geq \frac{\|g\|}{\Delta}-\|B\|_{1}=\lambda_{L}
$$

Agora, olhando a inequação à direita de (A.13) e observando que $\lambda_{1} \geq-\|B\|_{1}$, temos

$$
\lambda^{*} \leq \frac{\|g\|}{\left\|p\left(\lambda^{*}\right)\right\|}-\lambda_{1} \leq \frac{\|g\|}{\Delta}+\|B\|_{1}=\lambda_{U}
$$


Lema 1.2.5 Seja $B+\lambda I$ uma matriz definida positiva tal que $B+\lambda I=R^{T} R$. Para qualquer $\hat{z}$ tal que $\|\hat{z}\|=1$ vale que

$$
\lambda-\|R \hat{z}\|^{2} \leq-\lambda_{1}
$$

Prova Observe que

$$
\begin{gathered}
\|R \hat{z}\|^{2}=(R \hat{z})^{T}(R \hat{z})=\hat{z}^{T} R^{T} R \hat{z}=\hat{z}^{T}(B+\lambda I) \hat{z}= \\
\hat{z}^{T} B \hat{z}+\hat{z}^{T}(\lambda I) \hat{z}=\hat{z}^{T} B \hat{z}+\lambda \hat{z}^{T} \hat{z}=\hat{z}^{T} B \hat{z}+\lambda\|\hat{z}\|^{2} .
\end{gathered}
$$

Como $\|\hat{z}\|=1$, temos que

$$
\|R \hat{z}\|^{2}=\hat{z}^{T} B \hat{z}+\lambda \Rightarrow \lambda-\|R \hat{z}\|^{2}=-\hat{z}^{T} B \hat{z} .
$$

Tome a decomposição $B=Q \Lambda Q^{T}$, com $\Lambda=\operatorname{diag}\left(\lambda_{1}, \lambda_{2}, \ldots, \lambda_{n}\right), \lambda_{1} \leq \lambda_{2} \leq \ldots \leq \lambda_{n} \mathrm{e}$ $Q^{T} Q=I$. Temos então que

$$
\lambda-\|R \hat{z}\|^{2}=-\hat{z}^{T} Q \Lambda Q^{T} \hat{z}=-\hat{z}^{T} Q\left[\begin{array}{cccc}
\lambda_{1} & 0 & \cdots & 0 \\
0 & \lambda_{2} & \cdots & 0 \\
\vdots & \vdots & \ddots & \vdots \\
0 & 0 & \cdots & \lambda_{n}
\end{array}\right] Q^{T} \hat{z}
$$

Como $Q$ é ortogonal, temos

$$
\lambda-\|R \hat{z}\|^{2}=-\lambda_{1} \hat{z}_{1}^{2}-\lambda_{2} \hat{z}_{2}^{2}-\ldots-\lambda_{n} \hat{z}_{n}^{2},
$$

onde $\hat{z}_{i}$ é a i-ésima componente de $\hat{z}$.

Como $\lambda_{1} \leq \lambda_{j}$, para todo $j$, temos

$$
\lambda-\|R \hat{z}\|^{2} \leq-\lambda_{1}\left(\hat{z}_{1}^{2}+\hat{z}_{2}^{2}+\ldots+\hat{z}_{n}^{2}\right)=-\lambda_{1}\|\hat{z}\| .
$$

Mas $\|\hat{z}\|=1$. Portanto,

$$
\lambda-\|R \hat{z}\|^{2} \leq-\lambda_{1}
$$


Lema 1.2.6 Seja $\delta \geq 0$ tal que $B+\lambda I+\delta e_{l} e_{l}^{T}$ é singular, com $e_{l}$ a l-ésima coluna da matriz identidade. Seja $u \in \mathbb{R}^{n}$ tal que $\left(B+\lambda I+\delta e_{l} e_{l}^{T}\right) u=0$, com $u_{l}=1$ e $u_{i}=0$ para todo $i>l$. Então

$$
\lambda+\frac{\delta}{\|u\|^{2}} \leq-\lambda_{1}
$$

Prova Vamos calcular o quociente de Rayleigh $r(u)$ de $B$ (veja, por exemplo, [12]):

Como

$$
r(u)=\frac{u^{T} B u}{u^{T} u} .
$$

$$
\left(B+\lambda I+\delta e_{l} e_{l}^{T}\right) u=0 \Rightarrow B u=-\lambda u-\delta e_{l} e_{l}^{T} u .
$$

Assim,

$$
\begin{gathered}
r(u)=\frac{u^{T}\left(-\lambda u-\delta e_{l} e_{l}^{T} u\right)}{u^{T} u}=\frac{-\lambda u^{T} u-\delta u^{T} e_{l} e_{l}^{T} u}{u^{T} u}=-\lambda-\delta \frac{\left(e_{l}^{T} u\right)^{T}\left(e_{l}^{T} u\right)}{u^{T} u} \Rightarrow \\
r(u)=-\lambda-\delta \frac{\left\|e_{l}^{T} u\right\|^{2}}{\|u\|^{2}} .
\end{gathered}
$$

Mas, como $u_{l}=1$, temos $\left\|e_{l}^{T} u\right\|^{2}=1$. Ou seja,

$$
r(u)=-\lambda-\frac{\delta}{\|u\|^{2}} .
$$

Como $r(u) \geq \lambda_{1}$ para todo $u$, temos que

$$
-\lambda-\frac{\delta}{\|u\|^{2}} \geq \lambda_{1} \Rightarrow \lambda+\frac{\delta}{\|u\|^{2}} \leq-\lambda_{1} .
$$

Lema 1.2.7 Seja $0<\sigma<1$ dado e suponha que

$$
B+\lambda I=R^{T} R, \quad(B+\lambda I) p=-g, \quad \lambda \geq 0 .
$$

Se $\|p\| \geq(1-\sigma) \Delta$ então

$$
-\varphi(p) \geq \frac{1}{2}(1-\sigma)^{2}\left(\|R p\|^{2}+\lambda \Delta^{2}\right) \geq(1-\sigma)^{2}\left|\varphi^{*}\right|,
$$

onde $\varphi^{*}$ é o valor ótimo de (1.1). 
Prova Como a equação (A.10) vale para qualquer $z$, tome $z=0$

$$
\begin{gathered}
\varphi(p)=-\frac{1}{2}\left(\|R p\|^{2}+\lambda\|p\|^{2}\right) \Rightarrow-\varphi(p)=\frac{1}{2}\left(\|R p\|^{2}+\lambda\|p\|^{2}\right) \geq \\
\frac{1}{2}\left((1-\sigma)^{2}\|R p\|^{2}+\lambda\|p\|^{2}\right) .
\end{gathered}
$$

Por hipótese, temos que $\|p\| \geq(1-\sigma) \Delta$. Assim, temos que

$$
-\varphi(p) \geq \frac{1}{2}\left((1-\sigma)^{2}\|R p\|^{2}+\lambda((1-\sigma) \Delta)^{2}\right)=\frac{1}{2}(1-\sigma)^{2}\left(\|R p\|^{2}+\lambda \Delta^{2}\right) .
$$

Pelo mesmo raciocínio feito na prova do Lema 1.2.2, temos que

$$
\left|\varphi^{*}\right| \leq \frac{1}{2}\left(\|R p\|^{2}+\lambda \Delta^{2}\right) \Rightarrow(1-\sigma)^{2}\left|\varphi^{*}\right| \leq \frac{1}{2}(1-\sigma)^{2}\left(\|R p\|^{2}+\lambda \Delta^{2}\right) .
$$

Daí temos as duas desigualdades nas quais estamos interessados. 


\section{Referências Bibliográficas}

[1] E. G. Birgin e J. M. Martínez. A box-constrained optimization algorithm with negative curvature directions and spectral projected gradients. Computing [Suppl] 15, pp. 49-60, 2001.

[2] E. G. Birgin e J. M. Martínez. Large-scale active-set box-constrained optimization method with spectral projected gradients. Computational Optimization and Applications 23, pp. 101-125, 2002.

[3] E. G. Birgin, J. M. Martínez e M. Raydan. Inexact spectral projected gradient methods on convex sets. IMA Journal of Numerical Analysis 23, pp. 539-559, 2003.

[4] E. G. Birgin, J. M. Martínez e M. Raydan. Nonmonotone spectral projected gradient methods on convex sets. SIAM Journal on Optimization 10, pp. 1196-1211, 2000.

[5] E. G. Birgin, J. M. Martínez e M. Raydan. Algorithm 813: SPG - software for convexconstrained optimization. ACM Transactions on Mathematical Software 27, pp. 340-349, 2001.

[6] I. Bongartz, A. R. Conn, N. I. M. Gould e Ph. L. Toint. CUTE: constrained and unconstrained testing environment. ACM Transactions on Mathematical Software 21, pp. 123-160, 1995.

[7] A. K. Cline, C. B. Moler, G. W. Stewart e J. H. Wilkinson. An estimate for the condition number of a matrix. SIAM Journal on Numerical Analysis 16, pp. 368-375, 1979.

[8] A. R. Conn, N. I. M. Gould e Ph. L. Toint. A globally convergent Augmented Lagrangian algorithm for optimization with general constraints and simple bounds. SIAM Journal on Numerical Analysis 28, pp. 545-572, 1991.

[9] E. D. Dolan e J. J. Moré. Benchmarking optimization software with performance profiles. Mathematical Programming 91, pp. 201-213, 2002.

[10] R. Fletcher. Practical Methods of Optimization, Wiley, 1997.

[11] D. M. Gay. Computing optimal locally constrained steps. SIAM Journal on Scientific and Statistical Computing 2, pp. 186-197, 1981.

[12] G. H. Golub e C. F. van Loan. Matrix Computations, Johns Hopkins, 1996.

[13] J. M. Martínez e S. A. Santos. A trust-region strategy for minimization on arbitrary domains. Mathematical Programming 68, pp. 267-302, 1995. 
[14] J. M. Martínez e S. A. Santos. Convergence results on an algorithm for norm constrained regularization and related problems. RAIRO Operations Research 31, pp. 269-294, 1997.

[15] J. M. Martínez e S. A. Santos. Métodos Computacionais de Otimização, XX Colóquio Brasileiro de Matemática, IMPA, 1995.

[16] J. J. Moré. Recent developments in algorithms and software for trust-region methods. Em: A. Bachem, M. Grötschel e B. Korte, eds., Mathematical Programming Bonn 1982 - The State of Art, Springer-Verlag, 1983.

[17] J. J. Moré, B. S. Garbow e K. E. Hillstrom. Testing unconstrained optimization software. ACM Transactions on Mathematical Software 7, pp. 17-41, 1981.

[18] J. J. Moré e D. C. Sorensen. Computing a trust region step. SIAM Journal on Scientific and Statistical Computing 4, pp. 553-572, 1983.

[19] J. Nocedal e S. J. Wright. Numerical Optimization, Springer, 1999.

[20] M. Rojas, S. A. Santos e D. C. Sorensen. A new matrix-free algorithm for the large-scale trust-region subproblem. SIAM Journal on Optimization 11, pp. 611-646, 2000.

[21] L. Schrage. A more portable Fortran random number generator. ACM Transactions on Mathematical Software 5, pp. 132-138, 1979.

[22] D. C. Sorensen. Newton's method with a model trust-region modification. SIAM Journal on Numerical Analisis 19, pp. 409-426, 1982.

[23] S. W. Thomas. Sequential estimation techniques for quasi-Newton algorithms, Tese de Ph. D., Cornell University, Ithaca, NY, 1975. 